Algebraic 85 Geometric Topology

Volume 2 (2002) 1061-1118

Published: 25 November 2002

ATG

\title{
Common subbundles and intersections of divisors
}

\author{
N. P. StRickland
}

\begin{abstract}
Let $V_{0}$ and $V_{1}$ be complex vector bundles over a space $X$. We use the theory of divisors on formal groups to give obstructions in generalised cohomology that vanish when $V_{0}$ and $V_{1}$ can be embedded in a bundle $U$ in such a way that $V_{0} \cap V_{1}$ has dimension at least $k$ everywhere. We study various algebraic universal examples related to this question, and show that they arise from the generalised cohomology of corresponding topological universal examples. This extends and reinterprets earlier work on degeneracy classes in ordinary cohomology or intersection theory.
\end{abstract}

AMS Classification 55N20; 14L05,14M15

Keywords Vector bundle, divisor, degeneracy, Thom-Porteous, formal group

\section{Introduction}

There are a number of different motivations for the theory developed here, but perhaps the most obvious is as follows. Suppose we have a space $X$ with vector bundles $V_{0}$ and $V_{1}$. (Throughout this paper, the term "vector space" refers to finite-dimensional complex vector spaces equipped with Hermitian inner products, and similarly for "vector bundle".) We define the intersection index of $V_{0}$ and $V_{1}$ to be the largest $k$ such that $V_{0}$ and $V_{1}$ can be embedded isometrically in some bundle $U$ in such a way that $\operatorname{dim}\left(V_{0 x} \cap V_{1 x}\right) \geq k$ for all $x \in X$. We write $\operatorname{int}\left(V_{0}, V_{1}\right)$ for this intersection index. Our aim is to use invariants from generalised cohomology theory to estimate $\operatorname{int}\left(V_{0}, V_{1}\right)$, and to investigate the topology of certain universal examples related to this question.

We will show in Proposition 5.3 that $\operatorname{int}\left(V_{0}, V_{1}\right)$ is also the largest $k$ such that there is a linear map $V_{0} \rightarrow V_{1}$ of rank at least $k$ everywhere. This creates a link with the theory of degeneracy loci and the corresponding classes in the cohomology of manifolds or Chow rings of varieties, which are given by the determinantal formula of Thom and Porteous. The paper [9] by Pragacz is a convenient reference for comparison with the present work. The relevant theory 
is based strongly on Schubert calculus, and could presumably be transferred to complex cobordism (and thus to other complex-orientable theories) by the methods of Bressler and Evens [1].

However, our approach will be different in a number of ways. Firstly, we use the language of formal groups, as discussed in [10] (for example). We fix an even periodic cohomology theory $E$ with a complex orientation $x \in \widetilde{E}^{0} \mathbb{C} P^{\infty}$. For any space $X$ we have a formal scheme $X_{E}=\operatorname{spf}\left(E^{0} X\right)$, the basic examples being $S:=$ (point $)_{E}$ and $\mathbb{G}:=\mathbb{C} P_{E}^{\infty}=\operatorname{spf}\left(E^{0} \llbracket x \rrbracket\right)$, which is a formal group over $S$. If $V$ is a complex vector bundle over $X$, we write $P V$ for the associated bundle of projective spaces. It is standard that $E^{0}(P V)=E^{0}(X) \llbracket x \rrbracket / f_{V}(x)$, where $f_{V}(x)=\sum_{i+j=\operatorname{dim}(V)} c_{i} x^{j}$, where $c_{i}$ is the $i$ th Chern class of $V$. In geometric terms, this means that the formal scheme $D(V):=(P V)_{E}$ is naturally embedded as a divisor in $\mathbb{G} \times_{S} X_{E}$. Most of our algebraic constructions will have a very natural interpretation in terms of such divisors. We will also consider the bundle $U(V)=\coprod_{x \in X} U\left(V_{x}\right)$ of unitary groups associated to $V$. A key point is that $E^{*} U(V)$ is the exterior algebra over $E^{*} X$ generated by $E^{*-1} P V$. This provides a very natural link with exterior algebra, and could be regarded as the "real reason" for the appearance of determinantal formulae, which seem rather accidental in other approaches. Our divisorial approach also leads to descriptions of various cohomology rings that are manifestly independent of the choice of complex orientation, and depend functorially on $\mathbb{G}$. This functorality implicitly encodes the action of stable cohomology operations and thus gives a tighter link with the underlying homotopy theory.

We were also influenced by work of Kitchloo [5], in which he investigates the cohomological effect of Miller's stable splitting of $U(n)$, and draws a link with the theory of Schur functions.

In Section 3 we use the theory of Fitting ideals to define an intersection index $\operatorname{int}\left(D_{0}, D_{1}\right)$, where $D_{0}$ and $D_{1}$ are divisors on $\mathbb{G}$. In Section 4 we identify $E^{*} U(V)$ with the exterior algebra generated by $E^{*-1} P V$, and show that this identification is an isomorphism of Hopf algebras. In Section 5 we use this to prove our first main theorem, that $\operatorname{int}\left(V_{0}, V_{1}\right) \leq \operatorname{int}\left(D\left(V_{0}\right), D\left(V_{1}\right)\right)$; this implicitly gives all the relations among Chern classes that are universally satisfied when $\operatorname{int}\left(V_{0}, V_{1}\right) \geq k$ for some given integer $k$. Next, in Section 6 we study the universal examples of our various algebraic questions, focusing on the scheme $\operatorname{Int}_{r}\left(d_{0}, d_{1}\right)$ which classifies pairs $\left(D_{0}, D_{1}\right)$ of divisors of degrees $d_{0}$ and $d_{1}$ such that $\operatorname{int}\left(D_{0}, D_{1}\right) \geq k$. Our next task is to construct spaces whose associated schemes are these algebraic universal examples. In Section 7 we warm up by giving a divisorial account of the generalised cohomology of Grassmannians and 
flag spaces, and then in Section 8 we show that the space

$$
I_{r}^{\prime}\left(d_{0}, d_{1}\right):=\left\{\left(V_{0}, V_{1}\right) \in G_{d_{0}}\left(\mathbb{C}^{\infty}\right) \times G_{d_{1}}\left(\mathbb{C}^{\infty}\right) \mid \operatorname{dim}\left(V_{0} \cap V_{1}\right) \geq k\right\}
$$

satisfies $I_{r}^{\prime}\left(d_{0}, d_{1}\right)_{E}=\operatorname{Int}_{r}\left(d_{0}, d_{1}\right)$. (The origin of the present work is that the author needed to compute the cohomology of certain spaces similar to $I_{r}^{\prime}\left(d_{0}, d_{1}\right)$ as input to another project; it would take us too far afield to discuss the background.) This completes the main work of the paper, but we have added three more sections exploring the isomorphism $E^{*} U(V) \simeq \lambda^{*} E^{*-1} P V$ in more detail. Section 9 treats some purely algebraic questions related to this situation, and in Sections 10 and 11 we translate all the algebra into homotopy theory. In particular, this gives a divisorial interpretation of the work of Mitchell, Richter and others on filtrations of $\Omega U(n)$ : the scheme associated to the $k$ 'th stage in the filtration of $\Omega_{X} U(V)$ is $D(V)^{k} / \Sigma_{k}$, and the scheme associated to $\Omega_{X} U(V)$ is the free formal group over $X_{E}$ generated by $D(V)$.

Appendix A gives a brief treatment of the functional calculus for normal operators, which is used in a number of places in the text.

Remark 1.1 There is a theory of degeneracy loci for morphisms with symmetries, where the formulae involve Pfaffians instead of determinants. It would clearly be a natural project to reexamine this theory from the point of view of the present paper, but so far we have nothing to say about this.

\section{Notation and conventions}

\section{$2.1 \quad$ Spheres}

We take $\mathbb{R}^{n} \cup\{\infty\}$ as our definition of $S^{n}$, with $\infty$ as the basepoint; we distinguish $S^{1}$ from the homeomorphic space $U(1):=\{z \in \mathbb{C}|| z \mid=1\}$. Where necessary, we use the homeomorphism $\gamma: U(1) \rightarrow S^{1}$ given by

$$
\begin{aligned}
\gamma(z) & =(z+1)(z-1)^{-1} / i \\
\gamma^{-1}(t) & =(i t+1) /(i t-1) .
\end{aligned}
$$

One checks that $\gamma\left(e^{i \theta}\right)=\cot (-\theta / 2)$, which is a strictly increasing function of $\theta$ for $0<\theta<2 \pi$. 


\section{$2.2 \quad$ Fibrewise spaces}

We will use various elementary concepts from fibrewise topology; the book of Crabb and James [3] is a convenient reference. Very few topological technicalities arise, as our fibrewise spaces are all fibre bundles, and the fibres are usually finite complexes.

In particular, given spaces $U$ and $V$ over a space $X$, we write $U \times_{X} V$ for the fibre product, and $U_{X}^{n}$ for the fibre power $U \times \times_{X} \ldots \times_{X} U$. If $U$ is pointed (in other words, it has a specified section $s: X \rightarrow U$ ) and $E$ is any cohomology theory we write $\widetilde{E}_{X}^{*} U=E^{*}(U, s X)$. We also write $\Sigma_{X} U$ for the fibrewise suspension of $U$, which is the quotient of $S^{1} \times U$ in which $\{\infty\} \times U \cup S^{1} \times s X$ is collapsed to a copy of $X$. This satisfies $\widetilde{E}_{X}^{*} \Sigma_{X} U=\widetilde{E}_{X}^{*-1} U$. We also write $\Omega_{X} U$ for the fibrewise loop space of $U$, which is the space of maps $\omega: S^{1} \rightarrow U$ such that the composite $S^{1} \rightarrow U \rightarrow X$ is constant and $\omega(\infty) \in s X$. If $V$ is another pointed space over $X$, we write $U \wedge_{X} V$ for the fibrewise smash product. If $W$ is an unpointed space over $X$ then we write $W_{+X}=W \amalg X$, which is a pointed space over $X$ in an obvious way.

\subsection{Tensor products over schemes}

If $T$ is a scheme and $M, N$ are modules over the $\operatorname{ring} \mathcal{O}_{T}$, we will write $M \otimes_{T} N$ for $M \otimes_{\mathcal{O}_{T}} N$. Similarly, we write $\lambda_{T}^{k} M$ for $\lambda_{\mathcal{O}_{T}}^{k} M$, the $k$ 'th exterior power of $M$ over $\mathcal{O}_{T}$.

\subsection{Free modules}

Given a ring $R$ and a set $T$, we write $R\{T\}$ for the free $R$-module generated by $T$.

\section{$3 \quad$ Intersections of divisors}

Let $\mathbb{G}$ be a commutative, one-dimensional formal group over a scheme $S$. Choose a coordinate $x$ so that $\mathcal{O}_{\mathbb{G}}=\mathcal{O}_{S} \llbracket x \rrbracket$. Let $D_{0}$ and $D_{1}$ be divisors on $\mathbb{G}$ defined over $S$, with degrees $d_{0}$ and $d_{1}$ respectively. This means that $\mathcal{O}_{D_{i}}=\mathcal{O}_{\mathbb{G}} / f_{i}=\mathcal{O}_{S} \llbracket x \rrbracket / f_{i}(x)$ for some monic polynomial $f_{i}(x)$ of degree $d_{i}$ such that $f_{i}(x)=x^{d_{i}}$ modulo nilpotents. It follows that $\mathcal{O}_{D_{i}}$ is a free module of rank $d_{i}$ over $\mathcal{O}_{S}$, with basis $\left\{x^{j} \mid 0 \leq j<d_{i}\right\}$. 
As $D_{0}$ and $D_{1}$ are closed subschemes of $\mathbb{G}$ we can form their intersection, so that

$$
\mathcal{O}_{D_{0} \cap D_{1}}=\mathcal{O}_{\mathbb{G}} /\left(f_{0}, f_{1}\right)=\mathcal{O}_{S} \llbracket x \rrbracket /\left(f_{0}(x), f_{1}(x)\right) .
$$

Typically this will not be a projective module over $\mathcal{O}_{S}$, so some thought is required to give a useful notion of its size. We will use a measure coming from the theory of Fitting ideals, which we now recall briefly.

Let $R$ be a commutative Noetherian ring, and let $M$ be a finitely generated $R$-module. We can then find a presentation $P_{1} \stackrel{\phi_{1}}{\longrightarrow} P_{0} \stackrel{\phi_{0}}{\longrightarrow} M$, where $P_{0}$ and $P_{1}$ are finitely generated projective modules of ranks $p_{0}$ and $p_{1}$ say, and $M=\operatorname{cok}\left(\phi_{1}\right)$. The exterior powers $\lambda^{j} P_{i}$ are again finitely generated projective modules. We define $I_{j}\left(\phi_{1}\right)$ to be the smallest ideal in $R$ modulo which we have $\lambda^{j}\left(\phi_{1}\right)=0$. More concretely, if $P_{0}$ and $P_{1}$ are free then $\phi_{1}$ can be represented by a matrix $A$ and $I_{j}\left(\phi_{1}\right)$ is generated by the determinants of all $j \times j$ submatrices of $A$. We then define $I_{j}(M)=I_{p_{0}-j}\left(\phi_{1}\right)$; this is called the $j$ 'th Fitting ideal of $M$. It is a fundamental fact that this is well-defined; this was already known to Fitting (see [8, Chapter 3], for example), but we give a proof for the convenience of the reader.

Proposition 3.1 The ideal $I_{j}(M)$ is independent of the choice of presentation of $M$.

Proof We temporarily write $I_{j}\left(M, P_{*}, \phi_{*}\right)$ for the ideal called $I_{j}(M)$ above.

Put $N=\operatorname{ker}\left(\phi_{0}\right)$ and let $\beta: N \rightarrow P_{0}$ be the inclusion. Then $\phi_{1}$ factors as $P_{1} \stackrel{\alpha}{\rightarrow} N \stackrel{\beta}{\rightarrow} P_{0}$, where $\alpha$ is surjective. For any ideal $J \leq R$ we see that $\lambda^{k} \alpha$ is surjective $\bmod J$, so $\lambda^{k} \phi_{1}$ is zero $\bmod J$ iff $\lambda^{k} \beta$ is zero $\bmod J$. This condition depends only on the map $\phi_{0}: P_{0} \rightarrow M$, so we can legitimately define $I_{j}\left(M, P_{0}, \phi_{0}\right):=I_{j}\left(M, P_{*}, \phi_{*}\right)$.

Now suppose we have another presentation $Q_{1} \stackrel{\psi_{1}}{\longrightarrow} Q_{0} \stackrel{\psi_{0}}{\longrightarrow} M$, where $Q_{i}$ has rank $q_{i}$. Define $\chi_{0}: P_{0} \oplus Q_{0} \rightarrow M$ by $(u, v) \mapsto \phi_{0}(u)+\psi_{0}(v)$. It will suffice to prove that

$$
I_{j}\left(M, P_{0}, \phi_{0}\right)=I_{j}\left(M, P_{0} \oplus Q_{0}, \chi_{0}\right)=I_{j}\left(M, Q_{0}, \psi_{0}\right),
$$

and by symmetry we need only check the first of these. By projectivity we can choose a map $\theta: Q_{0} \rightarrow P_{0}$ with $\phi_{0} \theta=\psi_{0}$, and define $\chi_{1}: P_{1} \oplus Q_{0} \rightarrow P_{0} \oplus Q_{0}$ by $(u, v) \mapsto\left(\phi_{1}(u)-\theta(v), v\right)$. It is easy to check that this gives another presentation

$$
P_{1} \oplus Q_{0} \stackrel{\chi_{1}}{\longrightarrow} P_{0} \oplus Q_{0} \stackrel{\chi_{0}}{\longrightarrow} M .
$$


If $k \leq q_{0}$ then $\lambda^{k} \chi_{1}$ is certainly nonzero, because the composite

$$
\lambda^{k} Q_{0} \rightarrow \lambda^{k}\left(P_{1} \oplus Q_{0}\right) \stackrel{\lambda^{k} \chi}{\longrightarrow} \lambda^{k}\left(P_{0} \oplus Q_{0}\right) \rightarrow \lambda^{k} Q_{0}
$$

is the identity, and $\lambda^{k} Q_{0} \neq 0$. If $k>q_{0}$ and $\lambda^{k} \chi_{1}=0$ then (by restricting to $\left.\lambda^{k-q_{0}} P_{1} \otimes \lambda^{q_{0}} Q_{0}\right)$ we see that $\lambda^{k-q_{0}} \phi_{1}=0$.

For the converse, notice that $\lambda^{*} N$ is a graded ring for any module $N$, and that $\lambda^{*} \alpha$ is a ring map for any homomorphism $\alpha$ of $R$-modules. One can check that $\lambda^{j+q_{0}}\left(P_{1} \oplus Q_{0}\right)$ is contained in the ideal in $\lambda^{*}\left(P_{1} \oplus Q_{0}\right)$ generated by $\lambda^{j} P_{1}$. It follows that if $\lambda^{j} \phi_{1}=0$ then $\lambda^{j+q_{0}} \chi_{1}=0$.

This shows that $I_{r}\left(\phi_{1}\right)=I_{r+q_{0}}\left(\chi_{1}\right)$, and thus that $I_{r}\left(M, P_{0}, \phi_{0}\right)=I_{r}\left(M, P_{0} \oplus\right.$ $\left.Q_{0}, \chi_{0}\right)$, as required.

It is clear that

$$
I_{0}(M) \leq \ldots \leq I_{m}(M)=R
$$

and we define

$$
\operatorname{rank}(M)=\operatorname{rank}_{R}(M)=\min \left\{r \mid I_{r}(M) \neq 0\right\} .
$$

We call $\operatorname{rank}(M)$ the Fitting rank of $M$. For example, if $R$ is a principal ideal domain with fraction field $K$, one can check that $\operatorname{rank}(M)=\operatorname{dim}_{K}\left(K \otimes_{R} M\right)$ for all $M$. However, we will mostly be interested in rings $R$ with many nilpotents, for which there is no such simple formula.

The following lemma is easily checked from the definitions.

Lemma 3.2 (a) The Fitting rank is the same as the ordinary rank for projective modules.

(b) If $N$ is a quotient of $M$ then $\operatorname{rank}(N) \leq \operatorname{rank}(M)$.

(c) If there is a presentation $P \rightarrow Q \rightarrow M$ then $\operatorname{rank}(Q)-\operatorname{rank}(P) \leq$ $\operatorname{rank}(M) \leq \operatorname{rank}(Q)$.

(It is not true, however, that $\operatorname{rank}(M \oplus N)=\operatorname{rank}(M)+\operatorname{rank}(N)$; indeed, if $a \neq 0$ and $a^{2}=0$ then $\operatorname{rank}(R / a)=0$ but $\operatorname{rank}(R / a \oplus R / a)=1$.)

Definition 3.3 The intersection multiplicity of $D_{0}$ and $D_{1}$ is the integer

$$
\operatorname{int}\left(D_{0}, D_{1}\right):=\operatorname{rank}_{\mathcal{O}_{S}}\left(\mathcal{O}_{D_{0} \cap D_{1}}\right) \text {. }
$$

We also put

$$
\operatorname{Int}_{r}\left(D_{0}, D_{1}\right)=\operatorname{spec}\left(\mathcal{O}_{S} / I_{r-1}\left(\mathcal{O}_{D_{0} \cap D_{1}}\right)\right)
$$

which is the largest subscheme of $S$ over which we have $\operatorname{int}\left(D_{0}, D_{1}\right) \geq r$. 
Remark 3.4 Let $S^{\prime}$ be a scheme over $S$, so that $\mathbb{G}^{\prime}:=\mathbb{G} \times{ }_{S} S^{\prime}$ is a formal group over $S^{\prime}$. We refer to divisors on $\mathbb{G}^{\prime}$ as divisors on $\mathbb{G}$ over $S^{\prime}$. Given two such divisors $D_{0}$ and $D_{1}$, we get a closed subscheme $\operatorname{Int}_{r}\left(D_{0}, D_{1}\right) \subseteq S^{\prime}$. We will use this kind of base-change construction throughout the paper without explicit comment.

To make the above definitions more explicit, we will describe several different presentations of $\mathcal{O}_{D_{0} \cap D_{1}}$ that can be used to determine its rank.

Construction 3.5 First, recall that we can form the divisor

$$
D_{0}+D_{1}=\operatorname{spec}\left(\mathcal{O}_{\mathbb{G}} / f_{0} f_{1}\right)=\operatorname{spec}\left(\mathcal{O}_{S} \llbracket x \rrbracket / f_{0}(x) f_{1}(x)\right) .
$$

This contains $D_{0}$ and $D_{1}$, so we have a pullback square of closed inclusions as shown on the left below. This gives a pushout square of $\mathcal{O}_{S}$-algebras as shown on the right.
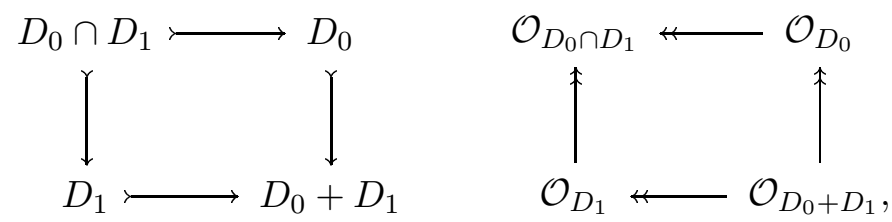

which gives a presentation

$$
\mathcal{O}_{D_{0}+D_{1}} \rightarrow \mathcal{O}_{D_{0}} \oplus \mathcal{O}_{D_{1}} \rightarrow \mathcal{O}_{D_{0} \cap D_{1}}
$$

Explicitly, this is just the presentation

$$
\mathcal{O}_{\mathbb{G}} /\left(f_{0} f_{1}\right) \stackrel{\phi}{\rightarrow} \mathcal{O}_{\mathbb{G}} / f_{0} \oplus \mathcal{O}_{\mathbb{G}} / f_{1} \stackrel{\psi}{\rightarrow} \mathcal{O}_{\mathbb{G}} /\left(f_{0}, f_{1}\right)
$$

given by

$$
\begin{aligned}
\phi\left(g \bmod f_{0} f_{1}\right) & =\left(g \bmod f_{0},-g \bmod f_{1}\right) \\
\psi\left(g_{0} \bmod f_{0}, g_{1} \bmod f_{1}\right) & =g_{0}+g_{1} \bmod \left(f_{0}, f_{1}\right) .
\end{aligned}
$$

Although this is probably the most natural presentation, it is not easy to write down the effect of $\phi$ on the obvious bases of $\mathcal{O}_{\mathbb{G}} /\left(f_{0} f_{1}\right)$ and $\mathcal{O}_{\mathbb{G}} / f_{i}$. To remedy this, we give an alternate presentation.

Construction 3.6 Let $J_{i}$ be the ideal generated by $f_{i}$ and put $J=J_{0} J_{1}$. Then $J_{i} / J$ is free over $\mathcal{O}_{S}$ with basis $\left\{x^{j} f_{i}(x) \mid 0 \leq j<d_{1-i}\right\}$ and the inclusion maps $J_{i} \rightarrow \mathcal{O}_{\mathbb{G}}$ give rise to a presentation

$$
J_{0} / J \oplus J_{1} / J \stackrel{\zeta}{\rightarrow} \mathcal{O}_{\mathbb{G}} / J=\mathcal{O}_{D_{0}+D_{1}} \stackrel{\xi}{\rightarrow} \mathcal{O}_{\mathbb{G}} /\left(J_{0}+J_{1}\right)=\mathcal{O}_{D_{0} \cap D_{1}} .
$$


Let $c_{i j}$ be the coefficient of $x^{d_{i}-j}$ in $f_{i}(x)$, so that $c_{i 0}=1$ and $f_{i}(x)=$ $\sum_{d_{i}=j+k} c_{i j} x^{k}$. Then

$$
\begin{aligned}
& \zeta\left(x^{j} f_{0}(x), 0\right)=\sum_{k=j}^{d_{0}+j} c_{0, d_{0}+j-k} x^{k} \quad \text { for } 0 \leq j<d_{1} \\
& \zeta\left(0, x^{j} f_{1}(x)\right)=\sum_{k=j}^{d_{1}+j} c_{1, d_{1}+j-k} x^{k} \quad \text { for } 0 \leq j<d_{0},
\end{aligned}
$$

and this tells us the matrix for $\zeta$ in terms of the obvious bases of $J_{0} / J \oplus J_{1} / J$ and $\mathcal{O}_{\mathbb{G}} / J$. For example, if $d_{0}=2$ and $d_{1}=3$ the matrix is

$$
\left(\begin{array}{ccc|cc}
c_{02} & 0 & 0 & c_{13} & 0 \\
c_{01} & c_{02} & 0 & c_{12} & c_{13} \\
1 & c_{01} & c_{02} & c_{11} & c_{12} \\
0 & 1 & c_{01} & 1 & c_{11} \\
0 & 0 & 1 & 0 & 1
\end{array}\right)
$$

In general, we have a square matrix with $d_{0}+d_{1}$ rows and columns. The left hand block consists of $d_{1}$ columns, each of which contains $d_{1}-1$ zeros. The right hand block consists of $d_{0}$ columns, each of which contains $d_{0}-1$ zeros. Clearly $\operatorname{Int}_{r}\left(D_{0}, D_{1}\right)$ is the closed subscheme defined by the vanishing of the minors of this matrix of size $d_{0}+d_{1}-r+1$. In particular, $\operatorname{Int}_{1}\left(D_{0}, D_{1}\right)$ is defined by the vanishing of the determinant of the whole matrix, which is classically known as the resultant of $f_{0}$ and $f_{1}$. If $f_{0}(x)=\prod_{i}\left(x-a_{i}\right)$ and $f_{1}(x)=\prod_{j}\left(x-b_{j}\right)$ then the resultant is just $\prod_{i, j}\left(a_{i}-b_{j}\right)$. We do not know of any similar formula for the other minors.

Construction 3.7 For a smaller but less symmetrical presentation, we can just use the sequence $J_{1} / J \rightarrow \mathcal{O}_{\mathbb{G}} / J_{0} \rightarrow \mathcal{O}_{\mathbb{G}} /\left(J_{0}+J_{1}\right)$ induced by the inclusion of $J_{1}$ in $\mathcal{O}_{\mathbb{G}}$. This is isomorphic to the presentation $\mathcal{O}_{\mathbb{G}} / J_{0} \stackrel{\mu_{1}}{\longrightarrow} \mathcal{O}_{\mathbb{G}} / J_{0} \rightarrow$ $\mathcal{O}_{\mathbb{G}} /\left(J_{0}+J_{1}\right)$, where $\mu_{1}(g)=f_{1} g$. However, the isomorphism depends on a choice of coordinate on $\mathbb{G}$ (because the element $f_{1}$ does), so the previous presentation is sometimes preferable. There is of course a similar presentation $\mathcal{O}_{\mathbb{G}} / J_{1} \stackrel{\mu_{0}}{\longrightarrow} \mathcal{O}_{\mathbb{G}} / J_{1} \rightarrow \mathcal{O}_{\mathbb{G}} /\left(J_{0}+J_{1}\right)$.

Finally, we give a presentation that depends only on the formal Laurent series $f_{0} / f_{1}$ and thus makes direct contact with the classical Thom-Porteous formula.

Construction 3.8 Write $\mathcal{M}_{G}=R((x))=\mathcal{O}_{\mathbb{G}}\left[x^{-1}\right]$. Note that $f_{1}(x) / x^{d_{1}}$ is a polynomial in $x^{-1}$ whose constant term is 1 and whose other coefficients are 
nilpotent, so it is a unit in $R\left[x^{-1}\right]$. It follows that $f_{1}$ is a unit in $R((x))$. Put $Q=x^{-1} R\left[x^{-1}\right] \subseteq R((x))$, so that $R((x))=R \llbracket x \rrbracket \oplus Q$. Multiplication by the series $x^{d_{1}} f_{0} / f_{1}$ induces a map

$$
P_{1}=\frac{R \llbracket x \rrbracket}{f_{1} R \llbracket x \rrbracket} \stackrel{\phi}{\rightarrow} P_{0}=\frac{R((x))}{x^{d_{1}} R \llbracket x \rrbracket \oplus Q} .
$$

We claim that the cokernel of $\phi$ is isomorphic to $R \llbracket x \rrbracket /\left(f_{0}, f_{1}\right)=\mathcal{O}_{D_{0} \cap D_{1}}$, so we have yet another presentation of this ring. Indeed, the cokernel of $\phi$ is clearly given by $R((x)) /\left(x^{d_{1}} f_{0} f_{1}^{-1} R \llbracket x \rrbracket+x^{d_{1}} R \llbracket x \rrbracket+Q\right)$. The element $f_{1} / x^{d_{1}}$ is invertible in $R\left[x^{-1}\right]$ so it is invertible in $R((x))$ and satisfies $\left(f_{1} / x^{d_{1}}\right) Q=Q$. Thus, multiplication by this element gives an isomorphism

$$
\frac{R((x))}{x^{d_{1}} f_{0} f_{1}^{-1} R \llbracket x \rrbracket+x^{d_{1}} R \llbracket x \rrbracket+Q} \simeq \frac{R((x))}{f_{0} R \llbracket x \rrbracket+f_{1} R \llbracket x \rrbracket+Q} .
$$

As $R((x))=R \llbracket x \rrbracket \oplus Q$, we see that the right hand side is just $R \llbracket x \rrbracket /\left(f_{0}, f_{1}\right)$ as claimed.

The elements $\left\{1, x, \ldots, x^{d_{1}-1}\right\}$ give a basis for both $P_{0}$ and $P_{1}$, and the matrix elements of $\phi$ with respect to these bases are just the coefficients of $f_{0} / f_{1}$ (suitably indexed). More precisely, we have

$$
f_{0} / f_{1}=x^{d_{0}-d_{1}} \sum_{i \geq 0} c_{i} x^{-i}
$$

where $c_{0}=1$ and $c_{i}$ is nilpotent for $i>0$. We take $c_{i}=0$ for $i<0$ by convention. The matrix elements $\Phi_{i j}$ of $\phi$ are then given by $\Phi_{i j}=c_{d_{0}+i-j}$ for $0 \leq i, j<d_{1}$. For example, if $d_{0}=3$ and $d_{1}=5$ then the matrix is

$$
\Phi=\left(\begin{array}{ccccc}
c_{3} & c_{4} & c_{5} & c_{6} & c_{7} \\
c_{2} & c_{3} & c_{4} & c_{5} & c_{6} \\
c_{1} & c_{2} & c_{3} & c_{4} & c_{5} \\
1 & c_{1} & c_{2} & c_{3} & c_{4} \\
0 & 1 & c_{1} & c_{2} & c_{3}
\end{array}\right) .
$$

Now suppose that our divisors $D_{i}$ arise in the usual way from vector bundles $V_{i}$ over a stably complex manifold $X$, and we have a generic linear map $g: V_{0} \rightarrow$ $V_{1}$. Let $Z_{r}$ be the locus where the rank of $g$ is at most $r$, and let $i: Z_{r} \rightarrow X$ be the inclusion. Generically, this will be a smooth stably complex submanifold of $X$, so we have a class $z_{r}=i_{*}\left[Z_{r}\right] \in E^{0} X$. The Thom-Porteous formula says that $z_{r}=\operatorname{det}\left(\Psi_{r}\right)$, where $\Psi_{r}$ is the square block of size $d_{1}-r$ taken from the bottom left of $\Phi$. More explicitly, the matrix elements are $\left(\Psi_{r}\right)_{i j}=c_{d_{0}-k+i-j}$ for $0 \leq i, j<d_{1}-r$. Clearly $\operatorname{det}\left(\Psi_{r}\right) \in I_{d_{1}-r}(\phi)=I_{r}\left(\mathcal{O}_{D_{0} \cap D_{1}}\right)$. If $Z_{r}$ is empty then $z_{r}=0$. On the other hand, Proposition 5.3 will tell us that 
$\operatorname{int}\left(D_{0}, D_{1}\right)>r$ and so $I_{r}\left(\mathcal{O}_{D_{0} \cap D_{1}}\right)=0$, so $\operatorname{det}\left(\Psi_{r}\right)=0$, which is consistent with the Thom-Porteous formula. It is doubtless possible to prove the formula using the methods of this paper, but we have not yet done so.

Proposition 3.9 We always have $\operatorname{int}\left(D_{0}, D_{1}\right) \leq \min \left(d_{0}, d_{1}\right)$ (unless the base scheme $S$ is empty). If $\operatorname{int}\left(D_{0}, D_{1}\right)=d_{0}$ then $D_{0} \leq D_{1}$, and if $\operatorname{int}\left(D_{0}, D_{1}\right)=d_{1}$ then $D_{1} \leq D_{0}$.

Proof The presentation $\mathcal{O}_{D_{1}} \stackrel{\mu_{0}}{\longrightarrow} \mathcal{O}_{D_{1}} \rightarrow \mathcal{O}_{D_{0} \cap D_{1}}$ shows that

$$
\operatorname{int}\left(D_{0}, D_{1}\right)=\operatorname{rank}\left(\mathcal{O}_{D_{0} \cap D_{1}}\right) \leq \operatorname{rank}\left(\mathcal{O}_{D_{1}}\right)=d_{1} .
$$

If this is actually an equality we must have $\lambda^{d_{1}-d_{1}+1}\left(\mu_{0}\right)=0$ or in other words $\mu_{0}=0$, so $f_{0}=0\left(\bmod f_{1}\right)$, so $D_{1} \leq D_{0}$. The remaining claims follow by symmetry.

Proposition 3.10 If there is a divisor $D$ of degree $k$ such that $D \leq D_{0}$ and $D \leq D_{1}$, then $\operatorname{int}\left(D_{0}, D_{1}\right) \geq k$.

Proof Clearly $\mathcal{O}_{D}$ is a quotient of the ring $\mathcal{O}_{D_{0} \cap D_{1}}$, and it is free of rank $k$, so $\operatorname{int}\left(D_{0}, D_{1}\right)=\operatorname{rank}\left(\mathcal{O}_{D_{0} \cap D_{1}}\right) \geq k$.

Definition 3.11 Given two divisors $D_{0}, D_{1}$, we write $\operatorname{Sub}_{r}\left(D_{0}, D_{1}\right)$ for the scheme of divisors $D$ of degree $r$ such that $D \leq D_{0}$ and $D \leq D_{1}$. The proposition shows that the projection $\pi: \operatorname{Sub}_{r}\left(D_{0}, D_{1}\right) \rightarrow S$ factors through the closed subscheme $\operatorname{Int}_{r}\left(D_{0}, D_{1}\right)$.

Remark 3.12 Proposition 3.9 implies that $\operatorname{Int}_{d_{0}}\left(D_{0}, D_{1}\right)$ is just the largest closed subscheme of $S$ over which we have $D_{0} \leq D_{1}$. From this it is easy to see that $\operatorname{Sub}_{d_{0}}\left(D_{0}, D_{1}\right)=\operatorname{Int}_{d_{0}}\left(D_{0}, D_{1}\right)$.

It is natural to expect that the map $\pi: \operatorname{Sub}_{r}\left(D_{0}, D_{1}\right) \rightarrow \operatorname{Int}_{r}\left(D_{0}, D_{1}\right)$ should be surjective in some suitable sense. Unfortunately this does not work as well as one might hope: the map $\pi$ is not faithfully flat or even dominant, so the corresponding ring map $\pi^{*}$ need not be injective. However, it is injective in a certain universal case, as we shall show in Section 6 .

We conclude this section with an example where $\pi^{*}$ is not injective. Let $\mathbb{G}$ be the additive formal group over the scheme $S=\operatorname{spec}\left(\mathbb{Z}[a] / a^{2}\right)$. Let $D_{0}$ and $D_{1}$ be the divisors with equations $x^{2}-a$ and $x^{2}$, respectively. Then $\mathcal{O}_{D_{0} \cap D_{1}}=\mathcal{O}_{S}[x] /\left(x^{2}-a, x^{2}\right)=\mathcal{O}_{S}[x] /\left(a, x^{2}\right)$, which is the cokernel of the map 
$\mu: \mathcal{O}_{S}[x] / x^{2} \rightarrow \mathcal{O}_{S}[x] / x^{2}$ given by $\mu(t)=a t$. The matrix of $\mu$ is $\left(\begin{array}{ll}a & 0 \\ 0 & a\end{array}\right)$ which is clearly nonzero, but $\lambda^{2}(\mu)=a^{2}=0$. It follows that $\operatorname{int}\left(D_{0}, D_{1}\right)=1$, so $\operatorname{Int}_{1}\left(D_{0}, D_{1}\right)=S$. However, $\operatorname{Sub}_{1}\left(D_{0}, D_{1}\right)$ is just the scheme $D_{0} \cap D_{1}=$ $\operatorname{spec}\left(\mathcal{O}_{S}[x] /\left(a, x^{2}\right)\right)$, so $\pi^{*}(a)=0$.

For a topological interpretation, let $V_{0}$ be the tautological bundle over $\mathbb{H} P^{1}=$ $S^{4}$, and let $V_{1}$ be the trivial rank two complex bundle. If we use the cohomology theory $E^{*} Y=\left(H^{*} Y\right)\left[u, u^{-1}\right]$ (with $|u|=2$ ) and let $a$ be the second Chern class of $V_{0}$ we find that $E^{0} X=\mathbb{Z}[a] / a^{2}$, and the equations of $D\left(V_{0}\right)$ and $D\left(V_{1}\right)$ are $x^{2}-a$ and $x^{2}$. Using the theory to be developed in Section 5 and the calculations of the previous paragraph, we deduce that $V_{0}$ and $V_{1}$ cannot have a common subbundle of rank one, but there is no cohomological obstruction to finding a map $f: V_{0} \rightarrow V_{1}$ with rank at least 1 everywhere. To see that such a map does in fact exist, choose a subspace $W<\mathbb{H}^{2}$ which is a complex vector space of dimension 2 , but not an $\mathbb{H}$-submodule. We can then take the constant bundle with fibre $\mathbb{H}^{2} / W$ as a model for $V_{1}$. The bundle $V_{0}$ is by definition a subbundle of the constant bundle with fibre $\mathbb{H}^{2}$, so there is an evident projection map $f: V_{0} \rightarrow V_{1}$. As $W$ is not an $\mathbb{H}$-submodule, we see that $f$ is nowhere zero and thus has rank at least one everywhere, as claimed.

\section{Unitary bundles}

In order to compare the constructions of the previous section with phenomena in topology, we need a topological interpretation of the exterior powers $\lambda^{k} \mathcal{O}_{D}$ when $D$ is the divisor associated to a vector bundle.

Let $V$ be a complex vector bundle of dimension $d$ over a space $X$. We can thus form a bundle $U(V)$ of unitary groups in the evident way (so $U(V)=$ $\left\{(x, g) \mid x \in X\right.$ and $\left.\left.g \in U\left(V_{x}\right)\right\}\right)$. The key point is that $E^{*} U(V)$ can be naturally identified with $\lambda_{E^{*} X}^{*} E^{*-1} P V$ (the exterior algebra over the ring $E^{*} X$ generated by the module $\left.E^{*} P V\right)$. Moreover, we can use the group structure on $U(V)$ to make $E^{*} U(V)$ into a Hopf algebra over $E^{*} X$, and we can make $\lambda_{E^{*} X}^{*} E^{*-1} P V$ into a Hopf algebra by declaring $E^{*} P V$ to be primitive. We will need to know that our isomorphism respects these structures. All this is of course well-known when $X$ is a point and $E$ represents ordinary cohomology. Kitchloo [5] has shown that if one chooses the right proof then the restriction on $E$ can be removed. With just a few more words, we will be able to remove the restriction on $X$ as well. 
We start by comparing $U(V)$ with a suitable classifying space. First let $V$ be a vector space rather than a bundle. We let $E U(V)$ denote the geometric realisation of the simplicial space $\left\{U(V)^{n+1}\right\}_{n \geq 0}$ and we put $B U(V)=E U(V) / U(V)$, which is the usual simplicial model for the classifying space of $U(V)$. There is a well-known map $\eta: U(V) \rightarrow \Omega B U(V)$, which is a weak equivalence of $H$-spaces. By adjunction we have a map $\zeta: \Sigma U(V) \rightarrow B U(V)$, which gives a map

$$
\zeta^{*}: \widetilde{E}^{*} B U(V) \rightarrow \widetilde{E}^{*} \Sigma U(V)=\widetilde{E}^{*-1} U(V) .
$$

The fact that $\eta$ is an $H$-map means that $\zeta$ is primitive, or in other words that

$$
\zeta \circ \mu=\zeta \circ\left(\pi_{0}+\pi_{1}\right) \in\left[\Sigma U(V)^{2}, B U(V)\right] .
$$

We can also construct a tautological bundle $T=E U(V) \times_{U(V)} V$ over $B U(V)$.

We now revert to the case where $V$ is a vector bundle over a space $X$, and perform all the above constructions fibrewise. Firstly, we construct the bundle $B U(V)=\left\{(x, e) \mid x \in X\right.$ and $\left.e \in B U\left(V_{x}\right)\right\}$. Note that each space $B U\left(V_{x}\right)$ has a canonical basepoint, and using these we get an inclusion $X \rightarrow B U(V)$.

A slightly surprising point is that there is a canonical homotopy equivalence $B U(V) \rightarrow X \times B U(d)$. Indeed, we can certainly perform the definition of $T$ fibrewise to get a tautological bundle over $B U(V)$, which is classified by a map $q: B U(V) \rightarrow B U(d)$, which is unique up to homotopy. We can combine this with the projection $p: B U(V) \rightarrow X$ to get a map $f=(p, q): B U(V) \rightarrow$ $X \times B U(d)$. The map $p$ is a fibre bundle projection, and the restriction of $q$ to each fibre of $p$ is easily seen to be an equivalence. It is now an easy exercise with the homotopy long exact sequence of $p$ to see that $f$ is a weak equivalence. (Nothing untoward happens with $\pi_{0}$ and $\pi_{1}$ because $B U(d)$ is simply connected.)

Remark 4.1 Let $q_{0}: X \rightarrow B U(d)$ be the restriction of $q$. Then $q_{0}$ classifies the bundle $\left.T\right|_{X} \simeq V$, so in general it will be an essential map. Thus, if we just use the basepoint of $B U(d)$ to make $X \times B U(d)$ into a based space over $X$, then our equivalence $f: B U(V) \simeq X \times B U(d)$ does not preserve basepoints, and cannot be deformed to do so. If it did preserve basepoints we could apply the fibrewise loop functor $\Omega_{X}$ and deduce that $U(V) \simeq X \times U(d)$, but this is false in general.

It follows from the above that $E^{*} B U(V)$ is a formal power series algebra over $E^{*} X$, generated by the Chern classes of $T$. It will be convenient for us to modify this description slightly by considering the virtual bundle $T-V$ (where $V$ is implicitly pulled back to $B U(V)$ by the map $p: B U(V) \rightarrow X)$. We 
have $f_{T}(t)=t^{d} \sum_{k=0}^{d} a_{k} t^{-k}$ and $f_{V}(t)=t^{d} \sum_{k=0}^{d} b_{k} t^{-k}$ for some coefficients $a_{k} \in E^{0} B U(V)$ and $b_{k} \in E^{0} X$ so $f_{T-V}(t)=f_{T}(t) / f_{V}(t)=\sum_{k \geq 0} c_{k} t^{-k}$ for some $c_{k} \in E^{0} B U(V)$. For $k \leq d$ we have $c_{k}=a_{k}\left(\bmod b_{1}, \ldots, b_{d}\right)$ and it follows easily that

$$
E^{*} B U(V)=\left(E^{*} X\right) \llbracket c_{1}, \ldots, c_{d} \rrbracket .
$$

Note that the restriction of $T-V$ to $X \subset B U(V)$ is trivial, so the classes $c_{k}$ restrict to zero on $X$.

Next, consider the fibrewise suspension $\Sigma_{X} U(V)$. By dividing each fibre into two cones we obtain a decomposition $\Sigma_{X} U(V)=C_{0} \cup C_{1}$ where the inclusion of $X$ in each $C_{i}$ is a homotopy equivalence, and $C_{0} \cap C_{1}=U(V)$. Using a MayerVietoris sequence we deduce that $\widetilde{E}_{X}^{*} \Sigma_{X} U(V) \simeq \widetilde{E}^{*-1} U(V)$ and that this can be regarded as an ideal in $E^{*} \Sigma_{X} U(V)$ whose square is zero. Moreover, the construction of $\zeta$ can be carried out fibrewise to get a map $\Sigma_{X} U(V) \rightarrow B U(V)$ which is again primitive. It follows that $\zeta$ induces a map

$$
\zeta^{*}: \operatorname{Ind}\left(E^{*} B U(V)\right) \rightarrow \operatorname{Prim}\left(E^{*-1} U(V)\right) .
$$

(Here Ind and Prim denote indecomposables and primitives over $E^{*} X$.) Note also that $\operatorname{Ind}\left(E^{*} B U(V)\right)$ is a free module over $E^{*} X$ generated by $\left\{c_{1}, \ldots, c_{d}\right\}$.

To prove that $\zeta^{*}$ is injective, we need to consider the complex reflection map $\rho: \Sigma_{X} P V_{+X} \rightarrow U(V)$, which we define as follows. For $t \in S^{1}=\mathbb{R} \cup\{\infty\}$ and $x \in X$ and $L \in P V_{x}$, the map $\rho(t, x, L)$ is the endomorphism of $V_{x}$ that has eigenvalue $\gamma^{-1}(t)$ on the line $L$, and eigenvalue 1 on $L^{\perp}$. Here $\gamma^{-1}(t)=$ $(i t+1) /(i t-1) \in U(1)$, as in Section 2.1. Using this we obtain a map $\xi=$ $\zeta \circ \Sigma_{X} \rho: \Sigma_{X}^{2} P V_{+X} \rightarrow B U(V)$.

Our next problem is to identify the virtual bundle $\xi^{*}(T-V)$ over $\Sigma_{X}^{2} P V_{+X}$. For this it is convenient to identify $S^{2}$ with $\mathbb{C} P^{1}$ and thus $\Sigma^{2} P V_{+X}$ with a quotient of $\mathbb{C} P^{1} \times P V$. We have tautological bundles $H$ and $L$ over $\mathbb{C} P^{1}$ and $P V$, whose Euler classes we denote by $y$ and $x$.

Lemma 4.2 We have $\xi^{*}(T-V) \simeq(H-1) \otimes L$. Moreover, there is a power series $g(s) \in E^{0} \llbracket s \rrbracket$ with $g(0)=1$ such that $\xi^{*} c_{k}=-y x^{k-1} g(x)$ for $k=$ $1, \ldots, d$. (If $E^{0}$ is torsion-free then $g(s)=1 / \log _{F}^{\prime}(x)$.)

Proof In the proof it will be convenient to write $T_{V}$ and $L_{V}$ instead of $T$ and $L$, to display the dependence on $V$.

First consider the case where $X$ is a point and $V=\mathbb{C}$. Then $\rho: S^{1} \rightarrow U(1)=$ $U(\mathbb{C})$ is a homeomorphism and $B U(\mathbb{C}) \simeq \mathbb{C} P^{\infty}$. It is a standard fact that 
$\xi: S^{2} \rightarrow B U(\mathbb{C})$ can be identified with the inclusion $\mathbb{C} P^{1} \rightarrow \mathbb{C} P^{\infty}$, and thus that $\xi^{*} T_{\mathbb{C}}=H$.

In the general case, note that we have a map $\xi_{L}: \mathbb{C} P^{1} \times P L \rightarrow B U(L)$ of spaces over $P V$. The projection $P L \rightarrow P V$ is a homeomorphism which we regard as the identity. If we let $\pi: P V \rightarrow X$ be the projection, we have a splitting $\pi^{*} V=L \oplus\left(\pi^{*} V \ominus L\right)$. The inclusion $L \rightarrow \pi^{*} V$ gives an inclusion $U(L) \rightarrow \pi^{*} U(V)$ and thus an inclusion $B U(L) \rightarrow \pi^{*} B U(V)$, or equivalently a map $\phi: B U(L) \rightarrow B U(V)$ covering $\pi$. As $T_{V}=V \times_{U(V)} E U(V)$ and $U(L)$ acts trivially on $\pi^{*} V \ominus L$ we see that $\phi^{*} T_{V}=T_{L} \oplus\left(\pi^{*} V \ominus L\right)$.

Next, we note that tensoring with $L$ gives an isomorphism $\tau: U(\mathbb{C}) \times P V \rightarrow$ $U(L)$ and thus an isomorphism $B \tau: B U(\mathbb{C}) \times P V \rightarrow B U(L)$ with $(B \tau)^{*} T_{L}=$ $T_{\mathbb{C}} \otimes L$.

One can check that the following diagram commutes:

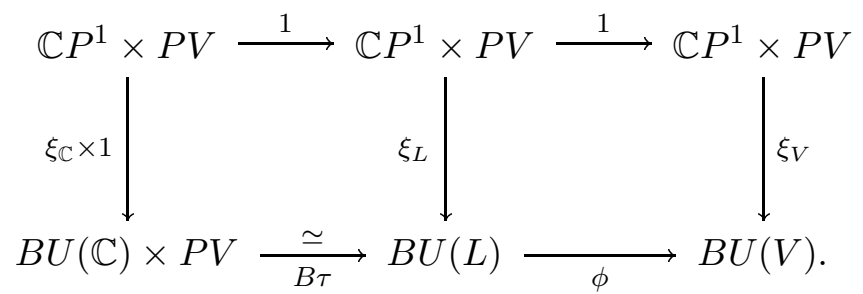

It follows that $\xi_{V}^{*} T_{V} \simeq\left(\xi_{\mathbb{C}} \times 1\right)^{*}(B \tau)^{*} \phi^{*} T_{V}$, and the previous discussion identifies this with $(H \otimes L) \oplus\left(\pi^{*} V \ominus L\right)$. It follows that $\xi_{V}^{*}\left(T_{V}-V\right) \simeq(H \otimes L)-L=$ $(H-1) \otimes L$, as claimed.

Now let $g(s)$ be the partial derivative of $t+{ }_{F} s$ with respect to $t$ evaluated at $t=0$. This is characterised by the equation $t+{ }_{F} s=t g(s)+s\left(\bmod t^{2}\right)$; it is clear that $g(0)=1$, and by applying $\log _{F}$ we see that $g(s)=1 / \log _{F}^{\prime}(s)$ in the torsion-free case. As $y^{2}=0$ we see that the Euler class of $H \otimes L$ is $x+{ }_{F} y=x+y g(x)$. Thus, we have

$$
\begin{aligned}
f_{H \otimes L-L}(t) & =(t-x-y g(x)) /(t-x) \\
& =1-y g(x) t^{-1} /(1-x / t) \\
& =1-\sum_{k>0} y g(x) x^{k-1} t^{-k} .
\end{aligned}
$$

The $k^{\prime}$ th Chern class of $(H-1) \otimes L$ is the coefficient of $t^{-k}$ in this series, which is $-y g(x) x^{k-1}$ as claimed.

Corollary 4.3 The induced map $\xi^{*}: \operatorname{Ind}\left(E^{*} B U(V)\right) \rightarrow E^{*}\left(\Sigma_{X}^{2} P V_{+X}, X\right)=$ $E^{*-2} P V$ is an isomorphism. 
Theorem 4.4 There is a natural isomorphism $\lambda^{*} E^{*-1} P V \rightarrow E^{*} U(V)$ of Hopf algebras over $E^{*} X$.

Proof Put $a_{i}=\xi^{*} c_{i} \in \operatorname{Prim}\left(E^{*} U(V)\right)$ for $i=1, \ldots, d$. Given a sequence $I=\left(i_{1}, \ldots, i_{r}\right)$ with $1 \leq i_{1}<\ldots<i_{r} \leq d$, put $a_{I}=\prod_{j} a_{i_{j}}$. We first claim that the elements $a_{I}$ form a basis for $E^{*} U(V)$ over $E^{*} X$. This is very well-known in the case where $X$ is a point (so $U(V) \simeq U(d)$ ) and $E$ represents ordinary cohomology; it can proved using the Serre spectral sequence of the fibration $U(d-1) \rightarrow U(d) \rightarrow S^{2 d-1}$. For a more general theory $E$ we still have an AtiyahHirzebruch-Serre spectral sequence $H^{p}\left(S^{2 d-1} ; E^{q} U(d-1)\right) \Longrightarrow E^{p+q} U(d)$. It follows easily that the elements $a_{I}$ form a basis whenever $X$ is a point. A standard argument now shows that they form a basis for any $X$. Indeed, it follows easily from the above that they form a basis whenever $V$ is trivialisable. We can give $X$ a cell structure such that $V$ is trivialisable over each cell, and then use Mayer-Vietoris sequences to check that the elements $a_{I}$ form a basis whenever $X$ is a finite complex. Finally, we can use the Milnor exact sequence to show that the elements $a_{I}$ form a basis for all $X$.

The ring $E^{*} U(V)$ is graded-commutative so we certainly have $a_{i} a_{j}=-a_{j} a_{i}$ and in particular $2 a_{i}^{2}=0$ for all $i$. Suppose we can show that $a_{i}^{2}=0$. Then $\zeta^{*}$ extends to give a map $\lambda_{E^{*} X}^{*} \operatorname{Ind}\left(E^{*} B U(V)\right) \rightarrow E^{*-1} U(V)$ of Hopf algebras, and from the previous paragraph we see that this is an isomorphism. Combining this with the isomorphism of Corollary 4.3 gives the required isomorphism $\lambda^{*} E^{*-1} P V \rightarrow E^{*} U(V)$.

All that is left is to check that $a_{i}^{2}=0$. For this we consider the case of the tautological bundle $T$ over $B U(d)$, and take $E=M P=M U\left[u, u^{-1}\right]$. (We use this 2-periodic version of $M U$ simply to comply with our standing assumptions on $E$; we could equally well use $M U$ itself.) Here it is standard that $M P^{*} B U(d)$ is a formal power series algebra over $M P^{*}$ and thus is torsionfree. The ring $M P^{*} U(T)$ is a free module over $M P^{*} B U(d)$ and thus is also torsion-free. As $2 a_{i}^{2}=0$ we must have $a_{i}^{2}=0$ as required. More generally, for an arbitrary bundle $V$ over a space $X$ we have a classifying map $X \rightarrow B U(d)$ giving rise to a map $U(V) \rightarrow U(T)$. Moreover, for any $E$ we can choose an orientation in degree zero and thus a ring map $M P \rightarrow E$. Together these give a ring map $M P^{*} U(T) \rightarrow E^{*} U(V)$, which carries $a_{i}$ to $a_{i}$. As $a_{i}^{2}=0$ in $M P^{*} U(T)$, the same must hold in $E^{*} U(V)$.

We will need to extend the above result slightly to give a topological interpretation of the quotient rings

$$
\lambda^{\leq r} E^{*-1} P V=\lambda^{*} E^{*-1} P V / \lambda^{>r} E^{*-1} P V .
$$


For this we recall Miller's filtration of $U(V)$ :

$$
\begin{aligned}
F_{k} U(V) & =\{g \in U(V) \mid \operatorname{codim}(\operatorname{ker}(g-1)) \leq k\} \\
& =\{g \in U(V) \mid \operatorname{rank}(g-1) \leq k\} .
\end{aligned}
$$

More precisely, this is supposed to be interpreted fibrewise, so

$$
F_{k} U(V)=\left\{(x, g) \mid x \in X \text { and } g \in U\left(V_{x}\right) \text { and } \operatorname{rank}(g-1) \leq k\right\} .
$$

It is not hard to see that $\rho$ gives a homeomorphism $\Sigma_{X} P V_{+X} \rightarrow F_{1} U(V)$. It is known from work of Miller [6] that when $X$ is a point, the filtration is stably split. Crabb showed in [2] that the splitting works fibrewise; our outline of related material essentially follows his account.

We will need to recall the basic facts about the quotients in Miller's filtration. Consider the space

$$
G_{k}(V)=\left\{(x, W) \mid x \in X, W \leq V_{x}, \operatorname{dim}(W)=k\right\} .
$$

For each point $(x, W) \in G_{k}(V)$ we have a Lie group $U(W)$ and its associated Lie algebra $\mathfrak{u}(W)=\left\{\alpha \in \operatorname{End}(W) \mid \alpha+\alpha^{*}=0\right\}$. These fit together to form a bundle over $G_{k}(V)$ which we denote by $\mathfrak{u}$. Given a point $(x, W, \alpha)$ in the total space of this bundle one checks that $\alpha-1$ is invertible and that $g:=(\alpha+1)(\alpha-1)^{-1}$ is a unitary automorphism of $W$ without fixed points, so $g \oplus 1_{W^{\perp}} \in F_{k} U\left(V_{x}\right) \backslash F_{k-1} U\left(V_{x}\right)$. It is not hard to show that this construction gives a homeomorphism of the total space of $\mathfrak{u}$ with $F_{k} U(V) \backslash F_{k-1} U(V)$ and thus a homeomorphism of the Thom space $G_{k}(V)^{\mathfrak{u}}$ with $F_{k} U(V) / F_{k-1} U(V)$. If $g \in F_{j} U\left(V_{x}\right)$ and $h \in F_{k} U\left(V_{x}\right)$ then $\operatorname{ker}(g-1) \cap \operatorname{ker}(h-1)$ has codimension at most $j+k$, so $g h \in F_{j+k} U(V)$, so the filtration is multiplicative. A less obvious argument shows that it is also comultiplicative, up to homotopy:

Lemma 4.5 The diagonal map $\delta: U(V) \rightarrow U(V) \times_{X} U(V)$ is homotopic to a filtration-preserving map.

Proof For notational convenience, we will give the proof for a vector space; it can clearly be done fibrewise for vector bundles.

We regard $U(1)$ as the set of unit complex numbers and define $p_{0}, p_{1}: U(1) \rightarrow$ $U(1)$ as follows:

$$
\begin{aligned}
& p_{0}(z)= \begin{cases}z^{2} & \text { if } \operatorname{Im}(z) \geq 0 \\
1 & \text { otherwise }\end{cases} \\
& p_{1}(z)= \begin{cases}z^{2} & \text { if } \operatorname{Im}(z) \leq 0 \\
1 & \text { otherwise }\end{cases}
\end{aligned}
$$


Thus $\left(p_{0}, p_{1}\right): U(1) \rightarrow U(1) \times U(1)$ is just the usual pinch map $U(1) \rightarrow U(1) \vee$ $U(1) \subset U(1) \times U(1)$.

Note that if $g \in U(V)$ and $r \in\{0,1\}$ then the eigenvalues of $g$ lie in $U(1)$ so we can interpret $p_{r}(g)$ as an endomorphism of $V$ as in Appendix A. As $p_{r}(U(1)) \subseteq U(1)$ we see that $\overline{p_{r}(z)}=p_{r}(z)^{-1}$ for all $z \in U(1)$ and thus that $p_{r}(g)^{*}=p_{r}(g)^{-1}$, so $p_{r}$ gives a map from $U(V)$ to itself.

We now define $\delta^{\prime}: U(V) \rightarrow U(V) \times U(V)$ by $\delta^{\prime}(g)=\left(p_{0}(g), p_{1}(g)\right)$. It is clear that the filtration of $p_{0}(g)$ is the number of eigenvalues of $g$ (counted with multiplicity) lying in the open upper half-circle, and the filtration of $p_{1}(g)$ is the number in the open lower half-circle. Thus, the filtration of $\delta^{\prime}(g)$ is the number of eigenvalues not equal to \pm 1 , which is less than or equal to the filtration of $g$.

On the other hand, each map $p_{r}: U(1) \rightarrow U(1)$ has degree 1 and thus is homotopic to the identity, so $\delta^{\prime}$ is homotopic to $\delta$.

Theorem 4.6 There is a natural isomorphism $\lambda_{E^{*} X}^{<k} E^{*-1} P V \rightarrow E^{*} F_{k-1} U(V)$.

Proof For brevity we write $\lambda^{k}=\lambda_{E^{*} X}^{k} E^{*-1} P V$. We also write $\lambda^{*}=\bigoplus_{k} \lambda^{k}$ and $\lambda^{\geq k}=\bigoplus_{j \geq k} \lambda^{j}$ and $\lambda^{<k}=\lambda^{*} / \lambda^{\geq k}=\bigoplus_{j<k} \lambda^{j}$.

Because the filtration of $U(V)$ is stably split, the restriction map $\lambda^{*} E^{*-1} P V=$ $E^{*} U(V) \rightarrow E^{*} F_{k-1} U(V)$ is a split surjection, with kernel $J_{k}$ say. Note that $\lambda^{*} / J_{k}$ and $J_{k}$ are both projective over $E^{*} X$. We need to show that $J_{k}=\lambda^{\geq k}$.

First, we have $F_{0} U(V)=X$ and it follows easily that $J_{1}=\lambda^{\geq 1}$.

We next claim that $J_{j} J_{k} \leq J_{j+k}$ for all $j, k$. Indeed, $J_{j}$ is the image in cohomology of the map $U(V) \rightarrow U(V) / F_{j-1}$, and so $J_{j} J_{k}$ is contained in the image in cohomology of the map

$$
\phi=\left(U(V) \stackrel{\delta}{\rightarrow} U(V) \times_{X} U(V) \rightarrow U(V) / F_{j-1} \wedge_{X} U(V) / F_{k-1}\right) .
$$

Note that $\delta$ is homotopic to the map $\delta^{\prime}$, which sends $F_{j+k-1}$ into $F_{j-1} \times_{X}$ $U(V) \cup U(V) \times_{X} F_{k-1}$. It follows that the restriction of $\phi$ to $F_{j+k-1}$ is null, and thus that $J_{j} J_{k} \leq J_{j+k}$ as claimed. It follows inductively that $\lambda^{\geq k} \leq J_{k}$ for all $k$. This gives us a natural surjective map $\lambda^{<k} \rightarrow E^{*} F_{k-1} U(V)$.

We previously gave a natural basis $\left\{a_{I}\right\}$ for $\lambda^{*}$, and it is clear that the subset $\left\{a_{I}|| I \mid<k\right\}$ is a basis for $\lambda^{<k}$. It will be enough to prove that the images of these form a basis for $E^{*} F_{k-1} U(V)$. The argument of Theorem 4.4 allows us to reduce to the case where $X$ is a point, $V=\mathbb{C}^{d}$, and $E$ represents 
ordinary cohomology. A proof in this case has been given by Kitchloo [5] (and possibly by others) but we will sketch an alternate proof for completeness. As the map $\lambda^{<k} \rightarrow H^{*} F_{k-1} U(d)$ is surjective, it will suffice to show that the source and target have the same rank as free Abelian groups. For this, it will suffice to show that $\lambda^{j} H^{*} \mathbb{C} P^{d-1}$ has the same rank as $\widetilde{H}^{*}\left(F_{j} U(d) / F_{j-1} U(d)\right)$ for $0 \leq j \leq d$. As $H^{*} \mathbb{C} P^{d-1}$ has rank $d$, it is clear that $\lambda^{j} H^{*} \mathbb{C} P^{d-1}$ has rank $\left(\begin{array}{c}d \\ j\end{array}\right)$. On the other hand $F_{j} U(d) / F_{j-1} U(d)$ is the Thom space $G_{j}\left(\mathbb{C}^{d}\right)^{\mathfrak{u}}$. Note that although $\mathfrak{u}$ is not a complex bundle, it is necessarily orientable because $G_{j}\left(\mathbb{C}^{d}\right)$ is simply connected. Thus, the Thom isomorphism theorem tells us that the rank of $\widetilde{H}^{*} G_{j}\left(\mathbb{C}^{d}\right)^{\mathfrak{u}}$ is the same as that of $H^{*} G_{j}\left(\mathbb{C}^{d}\right)$. By counting Schubert cells we see that this is again $\left(\begin{array}{c}d \\ j\end{array}\right)$, as required. (This will also follow from Proposition 7.3.)

\section{$5 \quad$ Intersections of bundles}

Let $X$ be a space, and let $V_{0}$ and $V_{1}$ be complex vector bundles over $X$. In Section 3 we defined divisors $D\left(V_{i}\right)=\left(P V_{i}\right)_{E}$ on $\mathbb{G}$ over $X_{E}$, and we also defined the intersection index $\operatorname{int}\left(V_{0}, V_{1}\right)$.

Theorem 5.1 We have $\operatorname{int}\left(V_{0}, V_{1}\right) \leq \operatorname{int}\left(D\left(V_{0}\right), D\left(V_{1}\right)\right)$.

Proof Suppose we have isometric linear embeddings $V_{0} \stackrel{j_{0}}{\longrightarrow} W \stackrel{j_{1}}{\longleftarrow} V_{1}$ such that $\operatorname{dim}\left(\left(j_{0} V_{0 x}\right) \cap\left(j_{1} V_{1 x}\right)\right) \geq r$ for all $x$. We must show that $\operatorname{rank}\left(\mathcal{O}_{\mathbb{G}} /\left(f_{V_{0}}, f_{V_{1}}\right)\right) \geq$ $r$. Put $d_{i}=\operatorname{dim}\left(V_{i}\right)$ and $e=\operatorname{dim}(W)$. Recall that $E^{0} P V_{i}=\mathcal{O}_{\mathbb{G}} / f_{V_{i}}$ and that $E^{0} P W=\mathcal{O}_{\mathbb{G}} / f_{W}$. As each $V_{i}$ embeds in $W$ we see that $f_{V_{i}}$ divides $f_{W}$ and there is a natural surjection $E^{0} P W \rightarrow E^{0} P V_{i}$. By combining these maps we get a map $\phi: E^{0} P W \rightarrow E^{0} P V_{0} \oplus E^{0} P V_{1}$, whose cokernel is $\mathcal{O}_{\mathbb{G}} /\left(f_{V_{0}}, f_{V_{1}}\right)$. From the definition of the Fitting rank, we must prove that $\lambda^{d_{0}+d_{1}-r+1} \phi=0$.

For this, we first note that an isometric embedding $j: V \rightarrow W$ of vector spaces gives rise to a homomorphism $j_{*}: U(V) \rightarrow U(W)$ by

$$
j_{*}(g)=j g j^{-1} \oplus 1_{j V^{\perp}}: W=j V \oplus j V^{\perp} \rightarrow W .
$$

The alternative description $j_{*}(g)=j g j^{*}+1-j j^{*}$ makes it clear that $j_{*}(g)$ depends continuously on $j$ and $g$. 
We now extend this definition fibrewise, and define $\gamma: U\left(V_{0}\right) \times{ }_{X} U\left(V_{1}\right) \rightarrow U(W)$ by $\gamma\left(g_{0}, g_{1}\right)=\left(j_{0 *} g_{0}\right)\left(j_{1 *} g_{1}\right)$. We have $E^{*} U(W)=\lambda^{*} E^{*-1} P W$ and

$$
\begin{aligned}
E^{*} U\left(V_{0}\right) \times_{X} U\left(V_{1}\right) & =E^{*} U\left(V_{0}\right) \otimes_{E^{*} X} E^{*} U\left(V_{1}\right) \\
& =\lambda^{*} E^{*-1} P V_{0} \otimes_{E^{*} X} \lambda^{*} E^{*-1} P V_{1} \\
& =\lambda^{*}\left(E^{*-1} P V_{0} \oplus E^{*-1} P V_{1}\right) .
\end{aligned}
$$

Using the fact that $E^{*-1} P W$ is primitive in $E^{*} U(W)$, we find that $\gamma^{*}=\lambda^{*} \phi$. Next, observe that if $g_{i} \in U\left(V_{i x}\right)$ for $i=0,1$ we have

$$
\gamma\left(g_{0}, g_{1}\right) \in U\left(j_{0} V_{0 x}+j_{1} V_{1 x}\right) \subseteq U(W)
$$

and $\operatorname{dim}\left(j_{0} V_{0 x}+j_{1} V_{1 x}\right) \leq d_{0}+d_{1}-r$ so $\gamma\left(g_{0}, g_{1}\right) \in F_{d_{0}+d_{1}-r} U(W)$. Thus $\gamma$ factors through $F_{d_{0}+d_{1}-r} U(W)$, and it follows that $\lambda^{d_{0}+d_{1}-r+1} E^{*-1} P W$ is mapped to zero by $\gamma^{*}$, as required.

As an addendum, we show that some natural variations of the definition of intersection index do not actually make a difference.

Lemma 5.2 Let $V$ and $W$ be vector bundles over a space $X$, and let $j: V \rightarrow$ $W$ be a linear embedding. Then $j$ is an isometric embedding if and only if $j^{*} j=1$ (where $j^{*}$ is the adjoint of $j$ ). In any case, there is a canonical isometric embedding $\hat{\jmath}: V \rightarrow W$ with the same image as $j$.

Proof If $j^{*} j=1$ then $\|j v\|^{2}=\langle j v, j v\rangle=\left\langle v, j^{*} j v\right\rangle=\langle v, v\rangle=\|v\|^{2}$, so $j$ is an isometry. Conversely, if $j$ is an isometry then it preserves inner products so $\left\langle v^{\prime}, j^{*} j v\right\rangle=\left\langle j v^{\prime}, j v\right\rangle=\left\langle v^{\prime}, v\right\rangle$ for all $v, v^{\prime}$ which means that $j^{*} j v=v$.

Even if $j$ is not an isometry we have $\left\langle v, j^{*} j v\right\rangle=\|j v\|^{2}$ which implies that $j^{*} j$ is injective. It is thus a strictly positive self-adjoint operator on $V$, so we can define $\left(j^{*} j\right)^{-1 / 2}$ by functional calculus (as in Appendix A). We then define $\hat{\jmath}=j \circ\left(j^{*} j\right)^{-1 / 2}$. This is the composite of $j$ with an automorphism of $V$, so it has the same image as $j$. It also satisfies $\hat{\jmath}^{*} \hat{\jmath}=1$, so it is an isometric embedding.

Proposition 5.3 Let $V_{0}$ and $V_{1}$ be bundles over a space $X$. Consider the following statements:

(a) There exists a bundle $V$ of dimension $k$ and linear isometric embeddings $V_{0} \stackrel{i_{0}}{\longleftarrow} V \stackrel{i_{1}}{\longrightarrow} V_{1}$.

$\left(\mathrm{a}^{\prime}\right)$ There exists a bundle $V$ of dimension $k$ and linear embeddings $V_{0} \stackrel{i_{0}}{\longleftarrow}$ $V \stackrel{i_{1}}{\rightarrow} V_{1}$. 
(b) There exist a bundle $W$ and isometric linear embeddings $V_{0} \stackrel{j_{0}}{\longrightarrow} W \stackrel{j_{1}}{\longleftarrow} V_{1}$ such that $\operatorname{dim}\left(\left(j_{0} V_{0 x}\right) \cap\left(j_{1} V_{1 x}\right)\right) \geq k$ for all $x \in X$.

$\left(\mathrm{b}^{\prime}\right)$ There exist a bundle $W$ and linear embeddings $V_{0} \stackrel{j_{0}}{\longrightarrow} W \stackrel{j_{1}}{\longleftarrow} V_{1}$ such that $\operatorname{dim}\left(\left(j_{0} V_{0 x}\right) \cap\left(j_{1} V_{1 x}\right)\right) \geq k$ for all $x \in X$.

(c) There is a linear map $f: V_{0} \rightarrow V_{1}$ such that $\operatorname{rank}\left(f_{x}\right) \geq k$ for all $x \in X$. Then $(a) \Leftrightarrow\left(a^{\prime}\right) \Rightarrow(b) \Leftrightarrow\left(b^{\prime}\right) \Leftrightarrow(c)$.

Proof It follows immediately from Lemma 5.2 that $(\mathrm{a}) \Leftrightarrow\left(\mathrm{a}^{\prime}\right)$ and $(\mathrm{b}) \Leftrightarrow\left(\mathrm{b}^{\prime}\right)$. (a) $\Rightarrow(\mathrm{b})$ : Define $W, j_{0}$ and $j_{1}$ by the following pushout square:

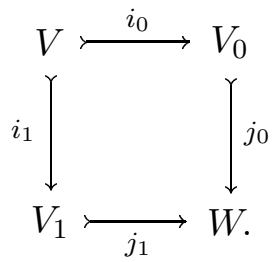

Equivalently, we can write $V_{t}^{\prime}$ for the orthogonal complement of $i_{t} V$ in $V_{t}$ and then $W=V \oplus V_{0}^{\prime} \oplus V_{1}^{\prime}$.

(b) $\Rightarrow(\mathrm{c})$ : Put $f=j_{1}^{*} j_{0}: V_{0} \rightarrow V_{1}$. By hypothesis, for each $x$ we can choose an orthonormal sequence $u_{1}, \ldots, u_{k}$ in $\left(j_{0} V_{0 x}\right) \cap\left(j_{1} V_{1 x}\right)$. We can then choose elements $v_{p} \in V_{0 x}$ and $w_{p} \in V_{1 x}$ such that $u_{p}=j_{0} v_{p}=j_{1} w_{p}$. We find that $\left\langle f v_{p}, w_{q}\right\rangle=\left\langle j_{0} v_{p}, j_{1} w_{q}\right\rangle=\left\langle u_{p}, u_{q}\right\rangle=\delta_{p q}$. This implies that the elements $f v_{1}, \ldots, f v_{k}$ are linearly independent, so $\operatorname{rank}(f) \geq k$ as required.

(c) $\Rightarrow(\mathrm{b})$ : Note that $f_{x}^{*} f_{x}: V_{0 x} \rightarrow V_{0 x}$ is a nonnegative self-adjoint operator with the same kernel as $f_{x}$, and thus the same rank as $f_{x}$. Similarly, $f_{x} f_{x}^{*}$ is a nonnegative self-adjoint operator on $V_{1 x}$ with the same rank as $f_{x}$. More basic facts about these operators are recorded in Proposition A.2.

As in Definition A.3 we let $\lambda_{j}=e_{j}\left(f_{x}^{*} f_{x}\right)$ be the $j$ 'th eigenvalue of $f_{x}^{*} f_{x}$ (listed in descending order and repeated according to multiplicity). We see from Proposition A.4 that $\lambda_{j}$ is a continuous function of $x$. Moreover, as $f_{x}^{*} f_{x}$ has rank at least $k$ we see that $\lambda_{k}>0$. Now define $\tau_{x}:[0, \infty) \rightarrow[0, \infty)$ by $\tau_{x}(t)=\max \left(\lambda_{k}, t\right)$, and define $\mu_{x}=\tau_{x}\left(f_{x}^{*} f_{x}\right)$ and $\nu_{x}=\tau\left(f_{x} f_{x}^{*}\right)$. (Here we are using functional calculus as in Appendix A again.) One checks that $f_{x} \mu_{x}=\nu_{x} f_{x}$ and $\mu_{x} f_{x}^{*}=f_{x}^{*} \nu_{x}$. We now have maps

$$
\begin{aligned}
\mu^{1 / 2} & : V_{0} \rightarrow V_{0} \\
f: & V_{0} \rightarrow V_{1} \\
\left(\mu+f^{*} f\right)^{-1 / 2}: & V_{0} \rightarrow V_{0},
\end{aligned}
$$


which we combine to get a map

$$
j_{0}=\left(\mu^{1 / 2}, f\right) \circ\left(\mu+f^{*} f\right)^{-1 / 2}: V_{0} \rightarrow V_{0} \oplus V_{1} .
$$

Similarly, we define

$$
j_{1}=\left(f^{*}, \nu^{1 / 2}\right) \circ\left(\nu+f f^{*}\right)^{-1 / 2}: V_{1} \rightarrow V_{0} \oplus V_{1} .
$$

It is easy to check that $j_{0}^{*} j_{0}=1$ and $j_{1}^{*} j_{1}=1$, so $j_{0}$ and $j_{1}$ are isometric embeddings.

Now choose an orthonormal sequence $v_{1}, \ldots, v_{k}$ of eigenvectors of $f_{x}^{*} f_{x}$, with eigenvalues $\lambda_{1}, \ldots, \lambda_{k}$. Put $v_{i}^{\prime}=f_{x}\left(v_{i}\right) / \sqrt{\lambda_{i}} \in V_{1}$; these vectors form an orthonormal sequence of eigenvectors of $f_{x} f_{x}^{*}$, with the same eigenvalues.

For $i \leq k$ we have $\lambda_{i} \geq \lambda_{k}>0$ so $\tau_{x}\left(\lambda_{i}\right)=\lambda_{i}$ so $\left(\mu+f^{*} f\right)^{-1 / 2}\left(v_{i}\right)=v_{i} / \sqrt{2 \lambda_{i}}$ and $\mu^{1 / 2}\left(v_{i}\right)=\sqrt{\lambda_{i}} v_{i}$ so $j_{0}\left(v_{i}\right)=\left(v_{i}, v_{i}^{\prime}\right) / \sqrt{2}$. This is the same as $j_{1}\left(v_{i}^{\prime}\right)$, so it lies in $\left(j_{0} V_{0 x}\right) \cap\left(j_{1} V_{1 x}\right)$. Thus, this intersection has dimension at least $k$, as required.

We conclude this section with a topological interpretation of the scheme $D\left(V_{0}\right) \cap$ $D\left(V_{1}\right)$ itself.

Proposition 5.4 Let $V_{0}$ and $V_{1}$ be vector bundles over a space $X$, and let $L_{0}$ and $L_{1}$ be the tautological bundles of the two factors in $P V_{0} \times_{X} P V_{1}$. Then there is a natural map $S\left(\operatorname{Hom}\left(L_{0}, L_{1}\right)\right)_{E} \rightarrow D\left(V_{0}\right) \cap D\left(V_{1}\right)$, which is an isomorphism if the map $E^{*} P\left(V_{0} \oplus V_{1}\right) \rightarrow E^{*} P V_{0} \oplus E^{*} P V_{1}$ is injective.

Proof We divide the sphere bundle $S\left(V_{0} \oplus V_{1}\right)$ into two pieces, which are preserved by the evident action of $U(1)$ :

$$
\begin{aligned}
& C_{0}=\left\{\left(v_{0}, v_{1}\right) \in S\left(V_{0} \oplus V_{1}\right) \mid\left\|v_{0}\right\| \geq\left\|v_{1}\right\|\right\} \\
& C_{1}=\left\{\left(v_{0}, v_{1}\right) \in S\left(V_{0} \oplus V_{1}\right) \mid\left\|v_{1}\right\| \geq\left\|v_{0}\right\|\right\} .
\end{aligned}
$$

The inclusions $V_{i} \rightarrow V_{0} \oplus V_{1}$ give inclusions $S\left(V_{i}\right) \rightarrow C_{i}$ which are easily seen to be homotopy equivalences. It follows that $C_{i} / U(1) \simeq P V_{i}$. We also have

$$
C_{0} \cap C_{1}=\left\{\left(v_{0}, v_{1}\right) \mid\left\|v_{0}\right\|=\left\|v_{1}\right\|=2^{-1 / 2}\right\} \simeq S\left(V_{0}\right) \times S\left(V_{1}\right) .
$$

Given a point in this space we have a map $\alpha: \mathbb{C} v_{0} \rightarrow \mathbb{C} v_{1}$ sending $v_{0}$ to $v_{1}$. This has norm 1 and is unchanged if we multiply $\left(v_{0}, v_{1}\right)$ by an element of $U(1)$. Using this we see that $\left(C_{0} \cap C_{1}\right) / U(1)=S\left(\operatorname{Hom}\left(L_{0}, L_{1}\right)\right)$. Of course, we also have $\left(C_{0} \cup C_{1}\right) / U(1)=P\left(V_{0} \oplus V_{1}\right)$. We therefore have a homotopy 
pushout square as shown on the left below, giving rise to a commutative square of formal schemes as shown on the right.
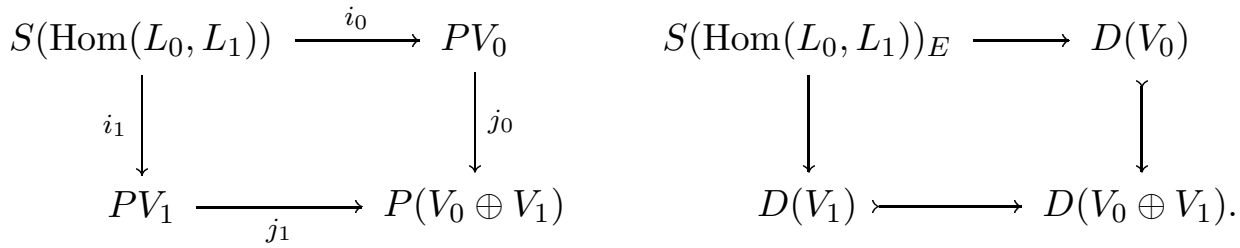

This evidently gives us a map $S\left(\operatorname{Hom}\left(L_{0}, L_{1}\right)\right)_{E} \rightarrow D\left(V_{0}\right) \cap D\left(V_{1}\right)$.

To be more precise, we use the Mayer-Vietoris sequence associated to our pushout square. This gives a short exact sequence

$$
\operatorname{cok}\left(f^{0}\right) \stackrel{p}{\rightarrow} E^{0} S\left(\operatorname{Hom}\left(L_{0}, L_{1}\right)\right) \stackrel{q}{\rightarrow} \operatorname{ker}\left(f^{-1}\right)
$$

where

$$
f^{k}=\left(j_{0}^{*}, j_{1}^{*}\right): E^{k} P\left(V_{0} \oplus V_{1}\right) \rightarrow E^{k} P V_{0} \oplus E^{k} P V_{1} .
$$

We have seen that $\operatorname{cok}\left(f^{0}\right)=\mathcal{O}_{D\left(V_{0}\right) \cap D\left(V_{1}\right)}$, and the map $p$ just corresponds to our map

$$
S\left(\operatorname{Hom}\left(L_{0}, L_{1}\right)\right)_{E} \rightarrow D\left(V_{0}\right) \cap D\left(V_{1}\right) .
$$

This map will thus be an isomorphism if $f^{*}$ is injective, as claimed.

\section{Algebraic universal examples}

Let $\mathbb{G}$ be a formal group over a formal scheme $S$. Later we will work with bundles over a space $X$, and we will take $S=X_{E}$ and $\mathbb{G}=\left(\mathbb{C} P^{\infty} \times X\right)_{E}$. We write $\operatorname{Div}_{d}^{+}=\operatorname{Div}_{d}^{+}(\mathbb{G}) \simeq \mathbb{G}^{d} / \Sigma_{d}$, so $\mathcal{O}_{\operatorname{Div}_{d}^{+}}=\mathcal{O}_{S} \llbracket c_{1}, \ldots, c_{d} \rrbracket$.

Fix integers $d_{0}, d_{1}, r \geq 0$. We write $\operatorname{Int}_{r}\left(d_{0}, d_{1}\right)$ for the scheme of pairs $\left(D_{0}, D_{1}\right)$ where $D_{0}$ and $D_{1}$ are divisors of degrees $d_{0}$ and $d_{1}$ on $\mathbb{G}$, and $\operatorname{int}\left(D_{0}, D_{1}\right) \geq r$. In other words, if $D_{i}$ is the evident tautological divisor over $\operatorname{Div}_{d_{0}}^{+} \times \operatorname{Div}_{d_{1}}^{+}$then $\operatorname{Int}_{r}\left(d_{0}, d_{1}\right)=\operatorname{Int}_{r}\left(D_{0}, D_{1}\right)$. We will assume that $r \leq \min \left(d_{0}, d_{1}\right)$ (otherwise we would have $\operatorname{Int}_{r}\left(d_{0}, d_{1}\right)=\emptyset$.)

For a more concrete description, put

$$
R=\mathcal{O}_{\operatorname{Div}_{d_{0}}^{+} \times \operatorname{Div}_{d_{1}}^{+}}=\mathcal{O}_{S} \llbracket c_{0 j}\left|j<d_{0} \rrbracket \llbracket c_{1 j}\right| j<d_{1} \rrbracket .
$$

Let $A$ be the matrix of $\zeta$ over $R$ as in Section 3, and let $I$ be the ideal in $R$ generated by the minors of $A$ of size $d_{0}+d_{1}-r+1$. Then $\operatorname{Int}_{r}\left(d_{0}, d_{1}\right)=$ $\operatorname{spf}(R / I)$. 
We will also consider a "semi-universal" case. Suppose we have a divisor $D_{1}$ on $\mathbb{G}$ over $S$, with degree $d_{1}$. Let $D_{0}$ be the tautological divisor over $\operatorname{Div}_{d_{0}}^{+}$. We can regard $D_{0}$ and $D_{1}$ as divisors on $\mathbb{G}$ over $\operatorname{Div}_{d_{0}}^{+}$and thus form the closed subscheme $\operatorname{Int}_{r}\left(D_{0}, D_{1}\right) \subseteq \operatorname{Div}_{d_{0}}^{+}$. We denote this scheme by $\operatorname{Int}_{r}\left(d_{0}, D_{1}\right)$.

We can also define schemes $\operatorname{Sub}_{r}\left(d_{0}, d_{1}\right)$ and $\operatorname{Sub}_{r}\left(d_{0}, D_{1}\right)$ in a parallel way.

Remark 6.1 $\operatorname{Sub}_{r}\left(d_{0}, d_{1}\right)$ is just the scheme of triples $\left(D, D_{0}, D_{1}\right)$ for which $D \leq D_{0}$ and $D \leq D_{1}$. This is isomorphic to the scheme of triples $\left(D, D_{0}^{\prime}, D_{1}^{\prime}\right) \in$ $\operatorname{Div}_{r}^{+} \times \operatorname{Div}_{d_{0}-r}^{+} \times \operatorname{Div}_{d_{1}-r}^{+}$, by the map $\left(D, D_{0}^{\prime}, D_{1}^{\prime}\right) \mapsto\left(D, D+D_{0}^{\prime}, D+D_{1}^{\prime}\right)$.

Definition 6.2 We write $\operatorname{Sub}_{r}(D)$ for the scheme of divisors $D^{\prime}$ of degree $r$ such that $D^{\prime} \leq D$. Using Remark 3.12 we see that $\operatorname{Sub}_{r}(D)=\operatorname{Sub}_{r}(r, D)=$ $\operatorname{Int}_{r}(r, D)$.

Theorem 6.3 The ring $\mathcal{O}_{\operatorname{Int}_{r}\left(d_{0}, d_{1}\right)}$ is freely generated over

$$
\mathcal{O}_{S} \llbracket c_{0 i}\left|0<i \leq d_{0}-r \rrbracket \llbracket c_{1 j}\right| 0<j \leq d_{1} \rrbracket
$$

by the monomials

$$
c_{0}^{\alpha}:=\prod_{i=d_{0}-r+1}^{d_{0}} c_{0 i}^{\alpha_{i}}
$$

for which $\sum_{i} \alpha_{i} \leq d_{1}-r$. Moreover, if we let $\pi: \operatorname{Sub}_{r}\left(d_{0}, d_{1}\right) \rightarrow \operatorname{Int}_{r}\left(d_{0}, d_{1}\right)$ be the usual projection, then the corresponding ring map $\pi^{*}$ is a split monomorphism of modules over $\mathcal{O}_{\operatorname{Div}_{d_{1}}^{+}}$(so $\pi$ itself is dominant).

The proof will be given after a number of intermediate results. It seems likely that the injectivity of $\pi^{*}$ could be extracted from work of Pragacz [9, Section 3]. He works with Chow groups of varieties rather than generalised cohomology rings of spaces, and his methods and language are rather different; we have not attempted a detailed comparison.

We start by setting up some streamlined notation. We put $n=d_{0}-r$ and $m=d_{1}-r$. We use the following names for the coordinate rings of various schemes of divisors, and the standard generators of these rings:

$$
\begin{aligned}
C_{0} & =\mathcal{O}_{\operatorname{Div}_{d_{0}}^{+}}=\mathcal{O}_{S} \llbracket u_{1}, \ldots, u_{n+r} \rrbracket \\
C_{1} & =\mathcal{O}_{\operatorname{Div}_{d_{1}}^{+}}=\mathcal{O}_{S} \llbracket v_{1}, \ldots, v_{m+r} \rrbracket \\
A & =\mathcal{O}_{\operatorname{Div}_{n}^{+}}=\mathcal{O}_{S} \llbracket a_{1}, \ldots, a_{n} \rrbracket \\
B & =\mathcal{O}_{\operatorname{Div}_{m}^{+}}=\mathcal{O}_{S} \llbracket b_{1}, \ldots, b_{m} \rrbracket \\
C & =\mathcal{O}_{\operatorname{Div}_{r}^{+}}=\mathcal{O}_{S} \llbracket c_{1}, \ldots, c_{r} \rrbracket .
\end{aligned}
$$


(In particular, we have renamed $c_{0 i}$ and $c_{1 i}$ as $u_{i}$ and $v_{i}$.) We put $u_{0}=v_{0}=$ $a_{0}=b_{0}=c_{0}=1$. We define $u_{i}=0$ for $i<0$ or $i>n+r$, and similarly for $v_{i}$, $a_{i}, b_{i}$ and $c_{i}$. The equations of the various tautological divisors are as follows:

$$
\begin{aligned}
f_{0}(x) & =\sum_{i} u_{i} x^{n+r-i} \in C_{0}[x] \\
f_{1}(x) & =\sum_{i} v_{i} x^{m+r-i} \in C_{1}[x] \\
f(x) & =\sum_{i} a_{i} x^{n-i} \in A[x] \\
g(x) & =\sum_{i} b_{i} x^{m-i} \in B[x] \\
h(x) & =\sum_{i} c_{i} x^{r-i} \in C[x] .
\end{aligned}
$$

We write $T_{0}$ for the set of monomials of weight at most $m$ in $u_{n+1}, \ldots, u_{n+r}$, and $T$ for the set of monomials of weight at most $m$ in $c_{1}, \ldots, c_{r}$. We also introduce the subrings

$$
\begin{aligned}
C_{0}^{\prime} & =\mathcal{O}_{S} \llbracket u_{1}, \ldots, u_{n} \rrbracket \subseteq C_{0} \\
C_{0}^{\prime \prime} & =\mathcal{O}_{S} \llbracket u_{1}, \ldots, u_{n-1} \rrbracket \subseteq C_{0}^{\prime} .
\end{aligned}
$$

We note that the ring $Q:=\mathcal{O}_{\operatorname{Int}_{r}\left(d_{0}, d_{1}\right)}$ has the form $\left(C_{0} \widehat{\otimes} C_{1}\right) / I$ for a certain ideal $I$. The theorem claims that $Q$ is freely generated as a module over $C_{0}^{\prime} \widehat{\otimes} C_{1}$ by $T_{0}$.

The map

$$
\pi^{*}: C_{0} \widehat{\otimes} C_{1} \rightarrow A \widehat{\otimes} B \widehat{\otimes} C
$$

sends $f_{0}(x)$ to $f(x) h(x)$ and $f_{1}(x)$ to $g(x) h(x)$. This induces a map $\pi^{*}: Q \rightarrow$ $A \widehat{\otimes} B \widehat{\otimes} C$, and the theorem also claims that this is a split injection.

We will need to approximate certain determinants by calculating their lowest terms with respect to a certain ordering. More precisely, we consider monomials of the form $u^{\alpha}=\prod_{i=1}^{n+r} u_{i}^{\alpha_{i}}$, and we order these by $u^{\alpha}<u^{\beta}$ if there exists $i$ such that $\alpha_{i}>\beta_{i}$ and $\alpha_{j}=\beta_{j}$ for $j>i$. The mnemonic is that $u_{1} \ll \ldots \ll u_{n+r}$, so any difference in the exponent of $u_{i}$ overwhelms any difference in the exponents of $u_{1}, \ldots, u_{i-1}$.

Lemma 6.4 Suppose we have integers $\gamma_{i}$ satisfying $0 \leq \gamma_{0}<\ldots<\gamma_{m}<$ $m+r$, and we put $M_{i j}=u_{n+r+i-\gamma_{j}}$ for $0 \leq i, j \leq m$, where $u_{k}$ is interpreted 
as 0 if $k<0$ or $k>n+r$. Then the lowest term in $\operatorname{det}(M)$ is the product of the diagonal entries, so

$$
\operatorname{det}(M)=\prod_{i=0}^{m} u_{n+r+i-\gamma_{i}}+\text { higher terms . }
$$

Remark 6.5 Determinants of this type are known as Schur functions.

Proof Put $\delta=\prod_{i=0}^{m} u_{n+r+i-\gamma_{i}}$. Let $M_{i}^{\prime}$ be obtained from $M$ by removing the 0 'th row and $i$ 'th column. The matrix $M_{0}^{\prime}$ has the same general form as $M$ so by induction we have $\operatorname{det}\left(M_{0}^{\prime}\right)=\prod_{i=1}^{m} u_{n+r+i-\gamma_{i}}+$ higher terms . If we expand $\operatorname{det}(M)$ along the top row then the 0 'th term is $u_{n+r-\gamma_{0}} \operatorname{det}\left(M_{0}^{\prime}\right)=$ $\delta+$ higher terms. As $0 \leq \gamma_{0}<\ldots<\gamma_{m}$ we have $\gamma_{i} \geq i+\gamma_{0}$ and so $\delta$ only involves variables $u_{j}$ with $j \leq n+r-\gamma_{0}$. The remaining terms in the row expansion of $\operatorname{det}(M)$ have the form $(-1)^{i} u_{n+r-\gamma_{0}+i} \operatorname{det}\left(M_{i}^{\prime}\right)$ for $i>0$, and $u_{n+r-\gamma_{0}+i}$ is either zero (if $i>\gamma_{0}$ ) or a variable strictly higher than all those appearing in $\delta$. The lemma follows easily.

Lemma 6.6 The ring $Q$ is generated by $T_{0}$ as a module over $C_{0}^{\prime} \widehat{\otimes} C_{1}$.

Proof Let $J$ be the ideal in $C_{0}^{\prime} \widehat{\otimes} C_{1}$ generated by $u_{1}, \ldots, u_{n}$ and $v_{1}, \ldots, v_{m+r}$, so $\left(C_{0}^{\prime} \widehat{\otimes} C_{1}\right) / J=\mathcal{O}_{S}$. We also put $C_{0}^{\prime \prime}=\left(C_{0} \widehat{\otimes} C_{1}\right) / J=\mathcal{O}_{S} \llbracket u_{n+1}, \ldots, u_{n+r} \rrbracket$. As $J$ is topologically nilpotent, it will suffice to prove the result modulo $J$. We will thus work modulo $J$ throughout the proof, so that $f_{1}=x^{m+r}$, and we must show that $Q / J$ is generated over $\mathcal{O}_{S}$ by $T_{0}$.

Let $\mu: C_{0}^{\prime \prime} \llbracket x \rrbracket / x^{m+r} \rightarrow C_{0}^{\prime \prime} \llbracket x \rrbracket / x^{m+r}$ be defined by $\mu(t)=f_{0} t$, and let $M$ be the matrix of $\mu$ with respect to the obvious bases. It is then easy to see that $Q / J=C_{0}^{\prime \prime} / I$, where $I$ is generated by the minors of $M$ of size $m+1$. The entries in $M$ are $M_{i j}=u_{n+r+i-j}$.

We next claim that all the generators $u_{k}$ are nilpotent mod $I$, or equivalently that $u_{k}=0$ in the ring $R=C_{0}^{\prime \prime} / \sqrt{I}$ for all $k$. By downward induction we may assume that $u_{l}=0$ in $R$ for $k<l \leq n+r$. We consider the submatrix $M^{\prime}$ of $M$ given by $M_{i j}^{\prime}=M_{i, n+r-k+j}=u_{i+k-j}$ for $0 \leq i, j \leq m$. By the definition of $I$ we have $\operatorname{det}\left(M^{\prime}\right) \in I$ and thus $\operatorname{det}\left(M^{\prime}\right)=0$ in $R$. On the other hand, we have $u_{l}=0$ for $l>k$ so $M^{\prime}$ is lower triangular so $\operatorname{det}\left(M^{\prime}\right)=\prod_{i} M_{i i}^{\prime}=u_{k}^{m+1}$. Thus $u_{k}$ is nilpotent in $R$ but clearly $\operatorname{Nil}(R)=0$ so $u_{k}=0$ in $R$ as required. It follows that $Q / J$ is a quotient of the polynomial ring $\mathcal{O}_{S}\left[u_{n+1}, \ldots, u_{n+r}\right] \subset$ $\mathcal{O}_{S} \llbracket u_{n+1}, \ldots, u_{n+r} \rrbracket$. 
Now let $W$ be the submodule of $Q / J$ spanned over $\mathcal{O}_{S}$ by $T_{0}$; we must prove that this is all of $Q / J$. As $1 \in W$, it will suffice to show that $W$ is an ideal. In the light of the previous paragraph, it will suffice to show that $W$ is closed under multiplication by the elements $u_{n+1}, \ldots, u_{n+r}$, or equivalently that $W$ contains all monomials of weight $m+1$.

We thus let $\alpha=\left(\alpha_{n+1}, \ldots, \alpha_{n+r}\right)$ be a multiindex of weight $m+1$. There is then a unique sequence $\left(\beta_{0}, \ldots, \beta_{m}\right)$ with $n+r \geq \beta_{0} \geq \ldots \geq \beta_{m}>n$ and $u^{\alpha}=\prod_{i} u_{\beta_{i}}$. Put $\gamma_{i}=n+r+i-\beta_{i}$, so that $0 \leq \gamma_{0}<\ldots<\gamma_{m}<m+r$. Let $M_{\alpha}$ be the submatrix of $M$ consisting of the first $m+1$ columns of the rows of indices $\gamma_{0}, \ldots, \gamma_{m}$, so the $(i, j)$ 'th entry of $M_{\alpha}$ is $u_{n+r+i-\gamma_{j}}$. Note that the elements $r_{\alpha}:=\operatorname{det}\left(M_{\alpha}\right)$ lie in $I$.

Lemma 6.4 tells us that the lowest term in $r_{\alpha}$ is $\prod_{i} u_{n+r+i-\gamma_{i}}=\prod_{i} u_{\beta_{i}}=u^{\alpha}$. It is clear that the weight of the remaining terms is at most the size of $M_{\alpha}$, which is $m+1$. By an evident induction, we may assume that their images in $C_{0}^{\prime \prime} / I$ lie in $W$. As $r_{\alpha} \in I$ we deduce that $u^{\alpha} \in W$ as well.

Corollary 6.7 Let $D_{1}$ be a divisor of degree $d_{1}$ on $\mathbb{G}$ over $S^{\prime}$, for some scheme $S^{\prime}$ over $S$. Then $\mathcal{O}_{\operatorname{Int}_{r}\left(d_{0}, D_{1}\right)}$ is generated over $\mathcal{O}_{S^{\prime}} \llbracket c_{01}, \ldots, c_{0, d_{0}-r} \rrbracket$ by the monomials $c_{0}^{\alpha}=\prod_{i=d_{0}-r+1}^{d_{0}} c_{0 i}^{\alpha_{i}}$ for which $|\alpha| \leq d_{1}-r$.

Proof The previous lemma is the universal case.

We next treat the special case of Theorem 6.3 where $n=0$ and so $r=d_{0}$. As remarked in Definition 6.2, the map $\pi: \operatorname{Sub}_{r}\left(d_{1}\right)=\operatorname{Sub}_{r}\left(r, d_{1}\right) \rightarrow \operatorname{Int}_{r}\left(r, d_{1}\right)$ is an isomorphism in this case.

Lemma 6.8 Let $D$ be a divisor of degree $d$ on $\mathbb{G}$ over $S$. For any $r \leq d$ we let $P_{r}(D)$ denote the scheme of tuples $\left(u_{1}, \ldots, u_{r}\right) \in \mathbb{G}^{r}$ such that $\sum_{i=1}^{r}\left[u_{r}\right] \leq D$. Then $\mathcal{O}_{P_{r}(D)}$ is free of rank $d ! /(d-r)$ ! over $\mathcal{O}_{S}$.

Proof There is an evident projection $P_{r}(D) \rightarrow P_{r-1}(D)$, which identifies $P_{r}(D)$ with the divisor $D-\left[u_{1}\right]-\ldots-\left[u_{r-1}\right]$ on $\mathbb{G}$ over $P_{r-1}(D)$. This divisor has degree $d-r+1$, so $\mathcal{O}_{P_{r}(D)}$ is free of rank $d-r+1$ over $\mathcal{O}_{P_{r-1}(D)}$. It follows by an evident induction that $\mathcal{O}_{P_{r}(D)}$ is free over $\mathcal{O}_{S}$, with rank $d(d-1) \ldots(d-r+1)=d ! /(d-r) !$.

Lemma 6.9 Let $D$ be a divisor of degree $d$ on $\mathbb{G}$ over $S$, let $D^{\prime}$ be the tautological divisor of degree $r$ over $\operatorname{Sub}_{r}(D)$, and let $f(x)=\sum_{i=0}^{r} c_{i} x^{r-i}$ be the equation of $D$. Then the set $T$ of monomials of degree at most $d-r$ in $c_{1}, \ldots, c_{r}$ is a basis for $\mathcal{O}_{\operatorname{Sub}_{r}(D)}$ over $\mathcal{O}_{S}$. 
Proof Put $K=|T|$; by elementary combinatorics we find that $K=\left(\begin{array}{c}d \\ r\end{array}\right)$. Put $R=\mathcal{O}_{\operatorname{Sub}_{r}(D)}$. Using $T$ we obtain an $\mathcal{O}_{S}$-linear map $\beta: \mathcal{O}_{S}^{K} \rightarrow R$, which is surjective by Lemma 6.6; we must prove that it is actually an isomorphism.

Now consider the scheme $P_{r}(D)$; Lemma 6.8 tells us that the ring $R^{\prime}:=\mathcal{O}_{P_{r}(D)}$ is a free module over $\mathcal{O}_{S}$ of rank $d ! /(d-r) !=r ! K$. On the other hand, $P_{r}(D)$ can be identified with the scheme of tuples $\left(D^{\prime}, u_{1}, \ldots, u_{r}\right)$ where $D^{\prime} \in \operatorname{Sub}_{r}(D)$ and $D^{\prime}=\left[u_{1}\right]+\ldots+\left[u_{r}\right]$. In other words, if we change base to $\operatorname{Sub}_{r}(D)$ we can regard $P_{r}(D)$ as $P_{r}\left(D^{\prime}\right)$, and now Lemma 6.8 tells us that $R^{\prime}$ is free of rank $r$ ! over $R$.

Now choose a basis $e_{1}, \ldots, e_{r}$ ! for $R^{\prime}$ over $R$. We can combine this with $\beta$ to get a map $\gamma: \mathcal{O}_{S}{ }^{r ! K} \rightarrow R^{\prime}$. This is a direct sum of copies of $\beta$, so it is surjective. Both source and target of $\gamma$ are free of rank $r ! K$ over $\mathcal{O}_{S}$. Any epimorphism between free modules of the same finite rank is an isomorphism (choose a splitting and then take determinants). Thus $\gamma$ is an isomorphism, and it follows that $\beta$ is an isomorphism as required.

Corollary 6.10 The set $T$ is a basis for $B \widehat{\otimes} C$ over $C_{1}$.

Proof This is the universal case of the lemma.

Corollary 6.11 The set $T$ is a basis for $A \widehat{\otimes} B \widehat{\otimes} C$ over $C_{0}^{\prime} \widehat{\otimes} C_{1}$.

Proof Note that $A \widehat{\otimes} B \widehat{\otimes} C=(B \widehat{\otimes} C) \llbracket a_{1}, \ldots, a_{n} \rrbracket$. For $0<i \leq n$ we have

$$
\pi^{*} u_{i}=\sum_{i=j+k} a_{j} c_{k}=a_{i}+c_{i} \bmod \text { decomposables, }
$$

where $c_{i}$ may be zero, but $a_{i}$ is nonzero. It follows that our ring $A \widehat{\otimes} B \widehat{\otimes} C$ can also be described as $(B \widehat{\otimes} C) \llbracket u_{1}, \ldots, u_{n} \rrbracket$, or equivalently as $C_{0}^{\prime} \widehat{\otimes} B \widehat{\otimes} C$. The claim now follows easily from the previous corollary.

Now let $T_{1}$ be the set of monomials of the form $u_{n}^{i} c_{1}^{\alpha_{1}} \ldots c_{n}^{\alpha_{n}}$ for which $0 \leq i<$ $|\alpha| \leq m$. These monomials can be regarded as elements of $A \widehat{\otimes} B \widehat{\otimes} C$, giving a map $\left(C_{0}^{\prime \prime} \widehat{\otimes} C_{1}\right)\left\{T_{1}\right\} \rightarrow A \widehat{\otimes} B \widehat{\otimes} C$. The map $\pi^{*}: C_{0} \widehat{\otimes} C_{1} \rightarrow A \widehat{\otimes} B \widehat{\otimes} C$ also gives us a map $\left(C_{0}^{\prime} \widehat{\otimes} C_{1}\right)\left\{T_{0}\right\} \rightarrow A \widehat{\otimes} B \widehat{\otimes} C$, and by combining these we get a map

$$
\phi:\left(C_{0}^{\prime} \widehat{\otimes} C_{1}\right)\left\{T_{0}\right\} \oplus\left(C_{0}^{\prime \prime} \widehat{\otimes} C_{1}\right)\left\{T_{1}\right\} \rightarrow A \widehat{\otimes} B \widehat{\otimes} C \simeq\left(C_{0}^{\prime} \widehat{\otimes} C_{1}\right)\{T\}
$$

of modules over $C_{0}^{\prime \prime} \widehat{\otimes} C_{1}$. Our main task will be to prove that this is an isomorphism. The proof will use the following lemma. 
Lemma 6.12 Let $R$ be a ring, and let $\alpha: M \rightarrow N$ be a homomorphism of modules over $R \llbracket x \rrbracket$. Suppose that $M$ can be written as a product of copies of $R \llbracket x \rrbracket$, and similarly for $N$. Suppose also that the induced map $M / x M \rightarrow$ $N / x N$ is an isomorphism. Then $\alpha$ is also an isomorphism.

Proof We have diagrams as shown below, in which the rows are easily seen to be exact:

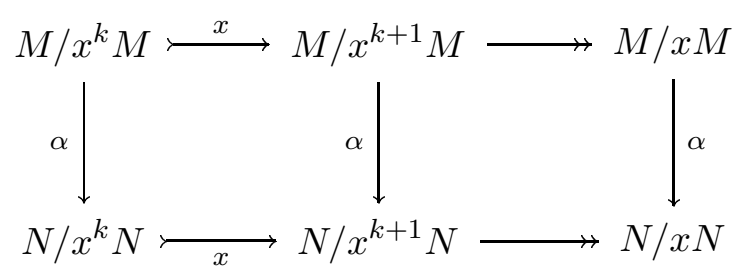

We see by induction that the maps $M / x^{k} M \rightarrow N / x^{k} N$ are all isomorphisms, and the claim follows by taking inverse limits.

Our map $\phi$ is a map of modules over the ring

$$
C_{0}^{\prime \prime} \widehat{\otimes} C_{1}=\mathcal{O}_{S} \llbracket u_{1}, \ldots, u_{n-1}, v_{1}, \ldots, v_{m+r} \rrbracket .
$$

Moreover, we have $C_{0}^{\prime} \widehat{\otimes} C_{1}=\left(C_{0}^{\prime \prime} \widehat{\otimes} C_{1}\right) \llbracket u_{n} \rrbracket \simeq \prod_{k=0}^{\infty} C_{0}^{\prime \prime} \widehat{\otimes} C_{1}$. Now let $J$ be the ideal in $C_{0}^{\prime \prime} \widehat{\otimes} C_{1}$ generated by $\left\{u_{1}, \ldots, u_{n-1}, v_{1}, \ldots, v_{m+r}\right\}$, so $\left(C_{0}^{\prime \prime} \widehat{\otimes} C_{1}\right) / J=$ $\mathcal{O}_{S}$ and $\phi$ induces a map

$$
\bar{\phi}: \mathcal{O}_{S} \llbracket u_{n} \rrbracket\left\{T_{0}\right\} \oplus \mathcal{O}_{S}\left\{T_{1}\right\} \rightarrow \mathcal{O}_{S} \llbracket u_{n} \rrbracket\{T\} .
$$

Note also that $\mathcal{O}_{S}\{T\}$ is the image of $C$ in $(A \widehat{\otimes} B \widehat{\otimes} C) / J$ and is thus a subring of $\mathcal{O}_{S} \llbracket u_{n} \rrbracket\{T\}$. By an evident inductive extension of the lemma, it will suffice to show that $\bar{\phi}$ is an isomorphism.

Lemma 6.13 We have $u_{n+j}=u_{n} c_{j}+w_{j}(\bmod J)$ for some polynomial $w_{j}$ in $c_{1}, \ldots, c_{r}$.

Proof For any monic polynomial $p(x)$ of degree $d$ we write $\hat{p}(y)=y^{d} p(1 / y)$. If $p(x)=\sum_{i} r_{i} x^{d-i}$ then $\hat{p}(y)=\sum_{i} r_{i} y^{i}$. Note that $\hat{p q}=\hat{p} \hat{q}$, and that $\hat{p}(0)=1$. As we work $\bmod \left(u_{i} \mid i<n\right)$ we have $\hat{f}_{0}=1\left(\bmod y^{n}\right)$. As we work $\bmod$ $\left(v_{j} \mid j \leq m+r\right)$ we have $\hat{f}_{1}=1$. We also have $f h=f_{0}$ and $g h=f_{1}$, so $\hat{f} \hat{h}=\hat{f}_{0}=1\left(\bmod y^{n}\right)$ and $\hat{g} \hat{h}=\hat{f}_{1}=1$. It follows easily that $\hat{f}=\hat{g}$ $\left(\bmod y^{n}\right)$, so $a_{i}=b_{i}$ for $i<n$.

We now have to distinguish between the case $m<n$ and the case $m \geq n$. First suppose that $m<n$. Then for $i>n$ we have $a_{i}=b_{i}=0$, and also $b_{n}=0$, and 
$a_{i}=b_{i}$ for $i<n$ by the previous paragraph. This implies that $\hat{f}-\hat{g}=a_{n} y^{n}$. We also have $(\hat{f}-\hat{g}) \hat{h}=\hat{f}_{0}-1$, and by comparing coefficients we deduce that $a_{n} c_{i}=u_{n+i}$ for $i=0, \ldots, r$. The case $i=0$ gives $u_{n}=a_{n}$, so $u_{n+i}=u_{n} c_{i}$ for $i=1, \ldots, r$, so the lemma is true with $w_{i}=0$.

Now suppose instead that $m \geq n$. As $a_{i}=0$ for $i>n$ we have

$$
\hat{f}-\hat{g}-\left(a_{n}-b_{n}\right) y^{n}=-\sum_{i=n+1}^{m} b_{i} y^{i} \in C[y] .
$$

We now multiply this by $\hat{h}$ and use the fact that $(\hat{f}-\hat{g}) \hat{h}=\hat{f}_{0}-1$. By comparing coefficients of $y^{n}$ we find that $u_{n}=a_{n}-b_{n}$. In view of this, our equation reads

$$
\hat{f}_{0}-1-u_{n} y^{n} \hat{h}=-\left(\sum_{i=n+1}^{m} b_{i} y^{i}\right) \hat{h} \in C[y] .
$$

The right hand side has the form $\sum_{j>0} w_{j} y^{n+j}$ with $w_{j} \in C$, and by comparing coefficients we see that $u_{n+j}=u_{n} c_{j}+w_{j}$ as claimed.

Proof of Theorem 6.3 Lemma 6.13 tells us that $\bar{\phi}\left(u^{\alpha}\right)$ is $u_{n}^{|\alpha|} c^{\alpha}$ plus terms involving lower powers of $u_{n}$. It follows easily that if we filter the source and target of $\bar{\phi}$ by powers of $u_{n}$, then the resulting map of associated graded modules is a isomorphism. It follows that $\bar{\phi}$ is an isomorphism, and thus that $\phi$ is an isomorphism. It follows that the map $\left(C_{0}^{\prime} \widehat{\otimes} C_{1}\right)\left\{T_{0}\right\} \rightarrow A \widehat{\otimes} B \widehat{\otimes} C$ is a split monomorphism of modules over $C_{0}^{\prime \prime} \widehat{\otimes} C_{1}$ (and thus certainly of modules over $\left.C_{1}\right)$. We have seen that this map factors as

$$
\left(C_{0}^{\prime} \widehat{\otimes} C_{1}\right)\left\{T_{0}\right\} \stackrel{\psi}{\rightarrow} Q \stackrel{\pi^{*}}{\longrightarrow} A \widehat{\otimes} B \widehat{\otimes} C,
$$

where $\psi$ is surjective by Lemma 6.6. It follows that $\psi$ is an isomorphism and that $\pi^{*}$ is a split monomorphism, as required.

\section{$7 \quad$ Flag spaces}

In the next section we will (in good cases) construct spaces whose associated formal schemes are the schemes $\operatorname{Sub}_{r}\left(D\left(V_{0}\right), D\left(V_{1}\right)\right)$ and $\operatorname{Int}_{r}\left(D\left(V_{0}\right), D\left(V_{1}\right)\right)$ considered previously. As a warm-up, and also as technical input, we will first consider the schemes associated to Grassmannian bundles and flag bundles. The results discussed are essentially due to Grothendieck [4]; we have merely adjusted the language and technical framework. 
Let $V$ be a bundle of dimension $d$ over a space $X$. We write $P_{r}(V)$ for the space of tuples $\left(x, L_{1}, \ldots, L_{r}\right)$ where $x \in X$ and $L_{1}, \ldots, L_{k} \in P V_{x}$ and $L_{i}$ is orthogonal to $L_{j}$ for $i \neq j$. Recall also that in Lemma 6.8 we defined $P_{r}(D(V))$ to be the scheme over $X_{E}$ of tuples $\left(u_{1}, \ldots, u_{r}\right) \in \mathbb{G}^{r}$ for which $\left[u_{1}\right]+\ldots+\left[u_{r}\right] \leq D(V)$.

Proposition 7.1 There is a natural isomorphism $P_{r}(V)_{E}=P_{r}(D(V))$.

Proof For each $i$ we have a line bundle over $P_{r}(V)$ whose fibre over the point $\left(x, L_{1}, \ldots, L_{r}\right)$ is $L_{i}$. This is classified by a map $P_{r}(V) \rightarrow \mathbb{C} P^{\infty}$, which gives rise to a map $u_{i}: P_{r}(V)_{E} \rightarrow \mathbb{G}$. The direct sum of these line bundles corresponds to the divisor $\left[u_{1}\right]+\ldots+\left[u_{r}\right]$. This direct sum is a subbundle of $V$, so $\left[u_{1}\right]+\ldots+\left[u_{r}\right] \leq D(V)$. This construction therefore gives us a map $P_{r}(V)_{E} \rightarrow P_{r}(D(V))$.

In the case $r=1$ we have $P_{1}(V)=P V$ and $P_{1}(D(V))=D(V)$ so the claim is that $(P V)_{E}=D(V)$, which is true by definition. In general, suppose we know that $P_{r-1}(V)_{E}=P_{r-1}(D(V))$. We can regard $P_{r}(V)$ as the projective space of the bundle over $P_{r-1}(V)$ whose fibre over a point $\left(x, L_{1}, \ldots, L_{r-1}\right)$ is the space $V_{x} \ominus\left(L_{1} \oplus \ldots \oplus L_{r-1}\right)$. It follows that $P_{r}(V)_{E}$ is just the divisor $D(V)-\left(\left[u_{1}\right]+\ldots+\left[u_{r-1}\right]\right)$ over $P_{r-1}(D(V))$, which is easily identified with $P_{r}(D(V))$. The proposition follows by induction.

Remark 7.2 One can easily recover the following more concrete statement. The ring $E^{0} P_{r}(V)=\mathcal{O}_{P_{r}(D(V))}$ is the largest quotient ring of $\left(E^{0} X\right) \llbracket x_{1}, \ldots, x_{r} \rrbracket$ in which the polynomial $f_{V}(t)$ is divisible by $\prod_{i=1}^{k}\left(t-x_{i}\right)$. It is a free module over $E^{0} X$ with rank $d ! /(d-r)$ !, and the monomials $x^{\alpha}$ with $0 \leq \alpha_{i} \leq d-i$ (for $i=1, \ldots, r$ ) form a basis. More details about the multiplicative relations are given in Section 9.

We next consider the Grassmannian bundle

$$
G_{r}(V)=\left\{(x, W) \mid x \in X, W \leq V_{x} \text { and } \operatorname{dim}(W)=r\right\} .
$$

Proposition 7.3 There is a natural isomorphism $G_{r}(V)_{E}=\operatorname{Sub}_{r}(D(V))$.

Proof Let $T$ denote the tautological bundle over $G_{r}(V)$. This is a rank $r$ subbundle of the pullback of $V$ so we have a degree $r$ subdivisor $D(T)$ of the pullback of $D(V)$ over $G_{r}(V)_{E}$. This gives rise to a map $G_{r}(V) \rightarrow \operatorname{Sub}_{r}(D(V))$.

Next, consider the space $P_{r}(V)$. There is a map $P_{r}(V) \rightarrow G_{r}(V)$ given by $\left(x, L_{1}, \ldots, L_{r}\right) \mapsto\left(x, L_{1} \oplus \ldots \oplus L_{r}\right)$. This lifts in an evident way to give a 
homeomorphism $P_{r}(V) \simeq P_{r}(T)$. Of course, this is exactly parallel to the proof of Lemma 6.9. Over $P_{r}(D(T))$ we have points $a_{1}, \ldots, a_{r}$ of $\mathbb{G}$ with coordinate values $x_{1}, \ldots, x_{r} \in \mathcal{O}_{P_{r}(D(T))}$ say. Let $B$ be the set of monomials $x^{\alpha}$ with $0 \leq \alpha_{i} \leq r-i$ for $i=1, \ldots, r$. From our earlier analysis of $\operatorname{Sub}_{r}(D(V))$ and $P_{r}(D(T))$ we see that $B$ is a basis for $\mathcal{O}_{P_{r}(D(T))}$ over $\mathcal{O}_{\operatorname{Sub}_{r}(D(V))}$. We also see from Remark 7.2 (applied to the bundle $T$ ) that $B$ is a basis for $E^{0} P_{r}(T)$ over $E^{0} G_{r}(V)$. This means that our isomorphism $f: \mathcal{O}_{P_{r}(D(V))} \rightarrow E^{0} P_{r}(V)$ is a direct sum (indexed by $B$ ) of copies of our map $g: \mathcal{O}_{\operatorname{Sub}_{r}(D(V))} \rightarrow E^{0} G_{r}(V)$. It follows that $g$ must also be an isomorphism.

Remark 7.4 Lemma 6.9 now gives us an explicit basis for $E^{0} G_{r}(V)$ over $E^{0} X$, consisting of monomials in the Chern classes of the tautological bundle $T$.

\section{Topological universal examples}

In this section we construct spaces whose associated formal schemes are the algebraic universal examples considered in Section 6.

We first consider the easy case of the schemes $\operatorname{Sub}_{r}\left(D_{0}, D_{1}\right)$.

Definition 8.1 Given vector bundles $V_{0}$ and $V_{1}$ over $X$, we define $G_{r}\left(V_{0}, V_{1}\right)$ to be the space of quadruples $\left(x, W_{0}, W_{1}, g\right)$ such that

(a) $x \in X$;

(b) $W_{i}$ is an $r$-dimensional subspace of $V_{i x}$ for $i=0,1$; and

(c) $g$ is an isometric isomorphism $W_{0} \rightarrow W_{1}$.

(We would obtain a homotopy equivalent space if we dropped the requirement that $g$ be an isometry.)

If $V_{i}$ is the evident tautological bundle over $B U\left(d_{i}\right)$ we write $G_{r}\left(d_{0}, d_{1}\right)$ for $G_{r}\left(V_{0}, V_{1}\right)$. More generally, if $V$ is a bundle over $X$ and $d_{0} \geq 0$ we can let $V_{1}$ be the pullback of $V$ to $B U\left(d_{0}\right) \times X$, and let $V_{0}$ be the pullback of of the tautological bundle over $B U\left(d_{0}\right)$; in this context we write $G_{r}\left(d_{0}, V\right)$ for $G_{r}\left(V_{0}, V_{1}\right)$.

Theorem 8.2 There is a natural map $p: G_{r}\left(V_{0}, V_{1}\right)_{E} \rightarrow \operatorname{Sub}_{r}\left(D\left(V_{0}\right), D\left(V_{1}\right)\right)$. In the universal case this is an isomorphism, so

$$
G_{r}\left(d_{0}, d_{1}\right)_{E}=\operatorname{Sub}_{r}\left(d_{0}, d_{1}\right) .
$$


More generally, there is a spectral sequence

$$
\operatorname{Tor}_{E^{*} B U\left(d_{0}\right) \times B U\left(d_{1}\right)}^{* *}\left(E^{*} X, E^{*} G_{r}\left(d_{0}, d_{1}\right)\right) \Longrightarrow E^{*} G_{r}\left(V_{0}, V_{1}\right),
$$

whose edge map in degree zero is the map

$$
p^{*}: \mathcal{O}_{\operatorname{Sub}_{r}\left(D\left(V_{0}\right), D\left(V_{1}\right)\right)} \rightarrow E^{0} G_{r}\left(V_{0}, V_{1}\right) .
$$

The spectral sequence collapses in the universal case. (We do not address the question of convergence in the general case.)

Proof First, we can pull back the bundles $V_{i}$ from $X$ to $G_{r}\left(V_{0}, V_{1}\right)$ (without change of notation). We also have a bundle over $G_{r}\left(V_{0}, V_{1}\right)$ whose fibre over a point $\left(x, W_{0}, W_{1}, g\right)$ is $W_{0}$; we denote this bundle by $W$, and note that there are natural inclusions $V_{0} \stackrel{1}{\leftarrow} W \stackrel{g}{\rightarrow} V_{1}$. We then have divisors $D(W)$ and $D\left(V_{i}\right)$ on $\mathbb{G}$ over $G_{r}\left(V_{0}, V_{1}\right)_{E}$ with $D(W) \leq D\left(V_{0}\right)$ and $D(W) \leq D\left(V_{1}\right)$, so the triple $\left(D\left(V_{0}\right), D\left(V_{1}\right), D(W)\right)$ is classified by a map $G_{r}\left(V_{0}, V_{1}\right)_{E} \rightarrow \operatorname{Sub}_{r}\left(D\left(V_{0}\right), D\left(V_{1}\right)\right)$.

We next consider the universal case. As our model of $E U(d)$ we use the space of orthonormal $d$-frames in $\mathbb{C}^{\infty}$, so $B U(d)$ is just the Grassmannian of $d$-planes in $\mathbb{C}^{\infty}$. Given a point

$$
(\underline{u}, \underline{v})=\left(u_{1}, \ldots, u_{d_{0}}, v_{1}, \ldots, v_{d_{1}}\right) \in E U\left(d_{0}\right) \times E U\left(d_{1}\right)
$$

we construct a point $\left(\left(V_{0}, V_{1}\right), W_{0}, W_{1}, g\right) \in G_{r}\left(d_{0}, d_{1}\right)$ as follows:

(a) $V_{0}$ is the span of $u_{1}, \ldots, u_{d_{0}}$

(b) $V_{1}$ is the span of $v_{1}, \ldots, v_{d_{1}}$

(c) $W_{0}$ is the span of $u_{1}, \ldots, u_{r}$

(d) $W_{1}$ is the span of $v_{1}, \ldots, v_{r}$

(e) $g$ is the map $W_{0} \rightarrow W_{1}$ that sends $u_{i}$ to $v_{i}$.

This gives a map $f: E U\left(d_{0}\right) \times E U\left(d_{1}\right) \rightarrow G_{r}\left(d_{0}, d_{1}\right)$. Next, the group $U\left(d_{0}\right) \times$ $U\left(d_{1}\right)$ has a subgroup $U(r) \times U\left(d_{0}-r\right) \times U(r) \times U\left(d_{1}-r\right)$, inside which we have the smaller subgroup $\Gamma$ consisting of elements of the form $\left(h, k_{0}, h, k_{1}\right)$. It is not hard to see that $\Gamma \simeq U(r) \times U\left(d_{0}-r\right) \times U\left(d_{1}-r\right)$, and that $f$ gives a homeomorphism $\left(E U\left(d_{0}\right) \times E U\left(d_{1}\right)\right) / \Gamma \rightarrow G_{r}\left(d_{0}, d_{1}\right)$. Moreover, $E U\left(d_{0}\right) \times$ $E U\left(d_{1}\right)$ is contractible and $\Gamma$ acts freely so $G_{r}\left(d_{0}, d_{1}\right) \simeq B \Gamma=B U(r) \times B U\left(d_{0}-\right.$ $r) \times B U\left(d_{1}-r\right)$, so $G_{r}\left(d_{0}, d_{1}\right)_{E}=\operatorname{Div}_{r}^{+} \times \operatorname{Div}_{d_{0}-r}^{+} \times \operatorname{Div}_{d_{1}-r}^{+}=\operatorname{Sub}_{r}\left(d_{0}, d_{1}\right)$ as claimed. 
In the general case we can choose maps $f_{i}: X \rightarrow B U\left(d_{i}\right)$ classifying $V_{i}$, and this gives rise to a pullback square as follows:

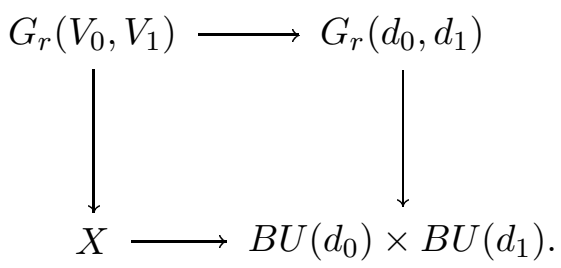

The vertical maps are fibre bundle projections so this is actually a homotopy pullback square. This give an Eilenberg-Moore spectral sequence as in the statement of the theorem. On the edge we have

$$
E^{*} X \otimes_{E^{*} B U\left(d_{0}\right) \times B U\left(d_{1}\right)} E^{*} G_{r}\left(d_{0}, d_{1}\right),
$$

which is the same as

$$
E^{*} X \otimes_{E^{0} B U\left(d_{0}\right) \times B U\left(d_{1}\right)} E^{*} G_{r}\left(d_{0}, d_{1}\right) .
$$

We can now identify this as the tensor product of $E^{*} X$ with $\mathcal{O}_{\operatorname{Sub}_{r}\left(d_{0}, d_{1}\right)}$ over $\mathcal{O}_{\operatorname{Div}_{d_{0}}^{+} \times \operatorname{Div}_{d_{1}}^{+}}$. The part in degree zero is easily seen to be $\mathcal{O}_{\operatorname{Sub}_{r}\left(D\left(V_{0}\right), D\left(V_{1}\right)\right)}$ as claimed.

We next show that our map $G_{r}\left(V_{0}, V_{1}\right)_{E} \rightarrow \operatorname{Sub}_{r}\left(D\left(V_{0}\right), D\left(V_{1}\right)\right)$ is an isomorphism in the semiuniversal case as well as the universal case. We start by analysing the semiuniversal spaces $G_{r}\left(d_{0}, V\right)$ in more familiar terms.

Proposition 8.3 There are natural homotopy equivalences

$$
G_{r}\left(d_{0}, V\right) \simeq G_{r}(V) \times B U\left(d_{0}-r\right)
$$

(and in particular $G_{r}(r, V) \simeq G_{r}(V)$ ).

Proof A point of $G_{r}\left(d_{0}, V\right)$ is a tuple $\left(V_{0}, x, W_{0}, W_{1}, g\right)$ where $V_{0} \in G_{d_{0}}\left(\mathbb{C}^{\infty}\right)$, $x \in X, W_{0} \in G_{r}\left(V_{0}\right), W_{1} \in G_{r}\left(V_{x}\right)$ and $g: W_{0} \rightarrow W_{1}$. We can define a map $f: G_{r}\left(d_{0}, V\right) \rightarrow G_{r}(V) \times B U\left(d_{0}-r\right)$ by $f\left(V_{0}, x, W_{0}, W_{1}, g\right)=\left(x, W_{1}, V_{0} \ominus W_{0}\right)$. It is not hard to see that this is a fibre bundle projection, and that the fibre over a point $\left(x, W, V^{\prime}\right)$ is the space of linear isometric embeddings from $W$ to $\mathbb{C}^{\infty} \ominus V^{\prime}$. This space is homeomorphic to the space of linear isometric embeddings of $\mathbb{C}^{r}$ in $\mathbb{C}^{\infty}$, which is well-known to be contractible. Thus $f$ is a fibration with contractible fibres and thus is a weak equivalence.

Corollary 8.4 The map $G_{r}\left(d_{0}, V\right)_{E} \rightarrow \operatorname{Sub}_{r}\left(d_{0}, D(V)\right)$ is an isomorphism. 
Proof Recall that $\operatorname{Sub}_{r}\left(d_{0}, D(V)\right)$ is the scheme of pairs $\left(D_{1}, D\right)$ where $D_{1}$ is a divisor of degree $d_{1}, D$ is a divisor of degree $r$ and $D \subseteq D_{1} \cap D(V)$. There is an evident isomorphism $\operatorname{Sub}_{r}(D(V)) \times_{S} \operatorname{Div}_{d_{0}-r}^{+} \rightarrow \operatorname{Sub}_{r}\left(d_{0}, D(V)\right)$ sending $\left(D^{\prime}, D\right)$ to $\left(D+D^{\prime}, D\right)$. The proposition tells us that $G_{r}\left(d_{0}, V\right)=$ $G_{r}(V) \times B U\left(d_{0}-r\right)$. We already know that $B U\left(d_{0}-r\right)_{E}=\operatorname{Div}_{d_{0}-r}^{+}$, and Proposition 7.3 tells us that $G_{r}(V)_{E}=\operatorname{Sub}_{r}(D(V))$. We therefore have an isomorphism $G_{r}\left(d_{0}, V\right)_{E}=\operatorname{Sub}_{r}(D(V)) \times{ }_{S} \operatorname{Div}_{d_{0}-r}^{+}=\operatorname{Sub}_{r}\left(d_{0}, D(V)\right.$ ). (This involves an implicit Künneth isomorphism, which is valid because $B U\left(d_{0}-r\right)$ has only even-dimensional cells.) We leave it to the reader to check that this isomorphism is the same as the map considered previously.

We now turn to parallel results for the schemes $\operatorname{Int}_{r}\left(D\left(V_{0}\right), D\left(V_{1}\right)\right)$.

Definition 8.5 Given vector bundles $V_{0}$ and $V_{1}$ over a space $X$, we define $I_{r}\left(V_{0}, V_{1}\right)$ to be the space of pairs $(x, f)$ where $f: V_{0 x} \rightarrow V_{1 x}$ is a linear map of rank at least $r$. We define the universal and semiuniversal spaces $I_{r}\left(d_{0}, d_{1}\right)$ and $I_{r}\left(d_{0}, V\right)$ by the evident analogue of Definition 8.1.

Remark 8.6 There is a natural map

$$
G_{r}\left(V_{0}, V_{1}\right) \rightarrow I_{r}\left(V_{0}, V_{1}\right),
$$

sending $\left(x, W_{0}, W_{1}, g\right)$ to $(x, f)$, where $f$ is the composite

$$
V_{0} \stackrel{\text { proj }}{\longrightarrow} W_{0} \stackrel{g}{\longrightarrow} W_{1} \stackrel{\text { inc }}{\longrightarrow} V_{1} .
$$

This gives a homeomorphism of $G_{r}\left(V_{0}, V_{1}\right)$ with the subspace of $I_{r}\left(V_{0}, V_{1}\right)$ consisting of pairs $(x, f)$ for which $f^{*} f$ and $f f^{*}$ are idempotent.

Definition 8.7 We define a natural map $q: I_{r}\left(V_{0}, V_{1}\right) \rightarrow \operatorname{Int}_{r}\left(D\left(V_{0}\right), D\left(V_{1}\right)\right)$ as follows. If we let $\pi$ denote the projection $I_{r}\left(V_{0}, V_{1}\right) \rightarrow X$ then we have a tautological map $f: \pi^{*} V_{0} \rightarrow \pi^{*} V_{1}$ which has rank at least $r$ everywhere. Proposition 5.3 now tells us that $\operatorname{int}\left(\pi^{*} V_{0}, \pi^{*} V_{1}\right) \geq r$. We can therefore apply Theorem 5.1 and deduce that the map $I_{r}\left(V_{0}, V_{1}\right)_{E} \rightarrow X_{E}$ factors through a map $q: I_{r}\left(V_{0}, V_{1}\right) \rightarrow \operatorname{Int}_{r}\left(D\left(V_{0}\right), D\left(V_{1}\right)\right) \subseteq X_{E}$ as required.

Later we will show that the map $q$ is an isomorphism in the universal case. For this, it will be convenient to have an alternative model for the universal space $I_{r}\left(d_{0}, d_{1}\right)$.

\section{Proposition 8.8 Put}

$$
I_{r}^{\prime}\left(d_{0}, d_{1}\right)=\left\{\left(V_{0}, V_{1}\right) \in G_{d_{0}}\left(\mathbb{C}^{\infty}\right) \times G_{d_{1}}\left(\mathbb{C}^{\infty}\right) \mid \operatorname{dim}\left(V_{0} \cap V_{1}\right) \geq k\right\} .
$$

Then $I_{r}^{\prime}\left(d_{0}, d_{1}\right)$ is homotopy equivalent to $I_{r}\left(d_{0}, d_{1}\right)$. 
Proof The basic idea is to refine the proof of Proposition 5.3. We will take $G_{d}\left(\mathbb{C}^{\infty}\right)$ as our model for $B U(d)$. We write $I=I_{r}\left(d_{0}, d_{1}\right)$ and $I^{\prime}=I_{r}^{\prime}\left(d_{0}, d_{1}\right)$ for brevity.

We will need various isometries between infinite-dimensional vector spaces. We define $\delta: \mathbb{C}^{\infty} \rightarrow \mathbb{C}^{\infty} \oplus \mathbb{C}^{\infty}$ by $\delta(v)=(v, v) / \sqrt{2}$, and we define $\theta: \mathbb{C}^{\infty} \oplus \mathbb{C}^{\infty} \rightarrow$ $\mathbb{C}^{\infty}$ by $\theta(v, w)=\left(v_{0}, w_{0}, v_{1}, w_{1}, \ldots\right)$. Next, it is well-known that the space of linear isometric embeddings of $\mathbb{C}^{\infty}$ in itself is contractible, so we can choose a continuous family of isometries $\phi_{t}$ with $\phi_{0}=\theta \delta$ and $\phi_{1}=1$. Similarly, we can choose continuous families of isometric embeddings $\psi_{0}^{t}, \psi_{1}^{t}: \mathbb{C}^{\infty} \rightarrow \mathbb{C}^{\infty} \oplus \mathbb{C}^{\infty}$ with $\psi_{0}^{0}(v)=\theta(v, 0)$ and $\psi_{1}^{0}(v)=\theta(0, v)$ and $\psi_{0}^{1}(v)=\psi_{1}^{1}(v)=v$.

We now define a map $\alpha: I^{\prime} \rightarrow I$ by $\alpha\left(V_{0}, V_{1}\right)=\left(V_{0}, V_{1}, f\right)$, where $f$ is the orthogonal projection map from $V_{0}$ to $V_{1}$. This acts as the identity on $V_{0} \cap V_{1}$ and thus has rank at least $k$. If we choose $n$ large enough that $V_{0}+V_{1} \leq \mathbb{C}^{n}$ and let $V_{0} \stackrel{i_{0}}{\longrightarrow} \mathbb{C}^{n} \stackrel{i_{1}}{\longleftarrow} V_{1}$ be the inclusions, then $f=i_{1}^{*} i_{0}$.

Next, we need to define a map $\beta: I \rightarrow I^{\prime}$. Given $\left(V_{0}, V_{1}, f\right) \in I$ we can construct maps

$$
\begin{aligned}
\mu: & V_{0} \rightarrow V_{0} \\
\nu: & V_{1} \rightarrow V_{1} \\
j_{0}: & V_{0} \rightarrow V_{0} \oplus V_{1}<\mathbb{C}^{\infty} \oplus \mathbb{C}^{\infty} \\
j_{1}: & V_{1} \rightarrow V_{0} \oplus V_{1}<\mathbb{C}^{\infty} \oplus \mathbb{C}^{\infty}
\end{aligned}
$$

as in the proof of the implication (c) $\Rightarrow$ (b) in Proposition 5.3, so $\operatorname{dim}\left(j_{0} V_{0} \cap\right.$ $\left.j_{1} V_{1}\right) \geq k$. We can thus define $\beta: I \rightarrow I^{\prime}$ by $\beta\left(V_{0}, V_{1}, f\right)=\left(\theta j_{0} V_{0}, \theta j_{1} V_{1}\right)$.

Suppose we start with $\left(V_{0}, V_{1}\right) \in I^{\prime}$, define $f: V_{0} \rightarrow V_{1}$ to be the orthogonal projection, and then define $j_{0}, j_{1}$ as above so that $\beta \alpha\left(V_{0}, V_{1}\right)=\left(\theta j_{0} V_{0}, \theta j_{1} V_{1}\right)$. Observe that $f^{*} f: V_{0} \rightarrow V_{0}$ decreases distances, and acts as the identity on $V:=V_{0} \cap V_{1}$. If we let $\lambda_{1}, \ldots, \lambda_{d_{0}}$ be the eigenvalues of $f^{*} f$ (listed in the usual way) we deduce that $\lambda_{1}=\ldots=\lambda_{k}=1$ and that $0 \leq \lambda_{i} \leq 1$ for all $i$. It follows from this that $\mu$ and $\nu$ are the respective identity maps, so

$$
\begin{aligned}
& j_{0}=(1, f) \circ\left(1+f^{*} f\right)^{-1 / 2} \\
& j_{1}=\left(f^{*}, 1\right) \circ\left(1+f f^{*}\right)^{-1 / 2} .
\end{aligned}
$$

In particular, we have $j_{0}(v)=j_{1}(v)=(v, v) / \sqrt{2}$ for $v \in V$, so $\left.j_{0}\right|_{V}=\left.j_{1}\right|_{V}=$ $\left.\delta\right|_{V}$.

Next, for $0 \leq t \leq 1$ we define $j_{0}^{t}: V_{0} \rightarrow \mathbb{C}^{\infty} \oplus \mathbb{C}^{\infty}$ by

$$
j_{0}^{t}=\left(i_{0}, t i_{0}+(1-t) f\right) \circ\left(1+t^{2}+\left(1-t^{2}\right) f^{*} f\right)^{-1 / 2} .
$$


One can check that this is an isometric embedding, with $j_{0}^{0}=j_{0}$ and $j_{0}^{1}=\left.\delta\right|_{V_{0}}$ and $\left.j_{0}^{t}\right|_{V}=\left.\delta\right|_{V}$ for all $t$. Similarly, if we put

$$
j_{1}^{t}=\left(i_{1}, t i_{0}+(1-t) f^{*}\right) \circ\left(1+t^{2}+\left(1-t^{2}\right) f f^{*}\right)^{-1 / 2},
$$

we find that this is an isometric embedding of $V_{1}$ in $\mathbb{C}^{\infty} \oplus \mathbb{C}^{\infty}$ with $j_{1}^{0}=j_{1}$ and $j_{1}^{1}=\left.\delta\right|_{V_{1}}$ and $\left.j_{1}^{t}\right|_{V}=\left.\delta\right|_{V}$ for all $t$. It follows that $\left(\theta j_{0}^{t} V_{0}, \theta j_{1}^{t} V_{1}\right) \in I^{\prime}$ for all $t$, and this gives a path from $\beta \alpha\left(V_{0}, V_{1}\right)=\left(\theta j_{0} V_{0}, \theta j_{1} V_{1}\right)$ to $\left(\theta \delta V_{0}, \theta \delta V_{1}\right)$. Recall that we chose a path $\left\{\phi_{t}\right\}$ from $\theta \delta$ to 1 . The pairs $\left(\phi_{t} V_{0}, \phi_{t} V_{1}\right)$ now give a path from $\left(\theta \delta V_{0}, \theta \delta V_{1}\right)$ to $\left(V_{0}, V_{1}\right)$ in $I^{\prime}$. Both of the paths considered above are easily seen to depend continuously on the point $\left(V_{0}, V_{1}\right) \in I^{\prime}$ that we started with, so we have constructed a homotopy $\beta \alpha \simeq 1$.

Now suppose instead that we start with a point $\left(V_{0}, V_{1}, f\right) \in I$; we need a path from $\alpha \beta\left(V_{0}, V_{1}, f\right)$ to $\left(V_{0}, V_{1}, f\right)$. We have $\beta\left(V_{0}, V_{1}, f\right)=\left(\theta j_{0} V_{0}, \theta j_{1} V_{1}\right)$, so $\alpha \beta\left(V_{0}, V_{1}, f\right)=\left(\theta j_{0} V_{0}, \theta j_{1} V_{1}, f^{\prime}\right)$, where $f^{\prime}: \theta j_{0} V_{0} \rightarrow \theta j_{1} V_{1}$ is the orthogonal projection. One can check that this is characterised by $f^{\prime}\left(\theta j_{0}(v)\right)=$ $\theta j_{1}\left(j_{1}^{*} j_{0}(v)\right)$. Next, for $0 \leq t \leq 1$ we define $k_{0}^{t}: V_{0} \rightarrow V_{0} \oplus V_{1}$ by

$$
k_{0}^{t}=\left(\sqrt{1-t^{2}+t^{2} \mu}, t f\right) \circ\left(1-t^{2}+t^{2} \mu+t^{2} f^{*} f\right)^{-1 / 2} .
$$

This is an isometric embedding with $k_{0}^{1}=j_{0}$ and $k_{0}^{0}(v)=(v, 0)$. Similarly, we define $k_{1}^{t}: V_{1} \rightarrow V_{0} \oplus V_{1}$ by

$$
k_{1}^{t}=\left(t f^{*}, \sqrt{1-t^{2}+t^{2} \nu}\right) \circ\left(1-t^{2}+t^{2} \nu+t^{2} f f^{*}\right)^{-1 / 2},
$$

and we define $f_{t}^{\prime}: \theta k_{0}^{t} V_{0} \rightarrow \theta k_{1}^{t} V_{1}$ by

$$
f_{t}^{\prime}\left(\theta k_{0}^{t}(v)\right)=\theta k_{1}^{t}\left(j_{1}^{*} j_{0}(v)\right),
$$

so $f_{1}^{\prime}=f^{\prime}$. The points $\left(k_{0}^{t} V_{0}, k_{1}^{t} V_{1}, f_{t}^{\prime}\right)$ give a path from $\alpha \beta\left(V_{0}, V_{1}, f\right)$ to $\left(\theta\left(V_{0} \oplus\right.\right.$ $\left.0), \theta\left(0 \oplus V_{1}\right), f_{0}^{\prime}\right)$ in $I$.

Next, we define $f_{t}^{\prime \prime}: \psi_{0}^{t} V_{0} \rightarrow \psi_{1}^{t} V_{1}$ by $f_{t}^{\prime \prime}\left(\psi_{0}^{t}(v)\right)=\psi_{1}^{t}\left(j_{1}^{*} j_{0}(v)\right)$. The points $\left(\psi_{0}^{t} V_{0}, \psi_{1}^{t} V_{1}, f_{t}^{\prime \prime}\right)$ give a path from $\left(\theta\left(V_{0} \oplus 0\right), \theta\left(0 \oplus V_{1}\right), f_{0}^{\prime}\right)$ to $\left(V_{0}, V_{1}, j_{1}^{*} j_{0}\right)$ in I. Using Proposition A.2 one can check that

$$
\begin{aligned}
j_{1}^{*} j_{0} & =\left(\nu+f f^{*}\right) \circ(f \sqrt{\mu}+\sqrt{\nu} f) \circ\left(\mu+f^{*} f\right) \\
& =f \circ\left(2 \mu^{1 / 2}\left(\mu+f^{*} f\right)^{-1}\right) .
\end{aligned}
$$

The map $\zeta:=2 \mu^{1 / 2}\left(\mu+f^{*} f\right)^{-1}$ is a strictly positive self-adjoint automorphism of $V_{0}$, so the same is true of $t+(1-t) \zeta$ for $0 \leq t \leq 1$. The points $\left(V_{0}, V_{1}, f \circ\right.$ $(t+(1-t) \zeta))$ form a path from $\left(V_{0}, V_{1}, j_{1}^{*} j_{0}\right)$ to $\left(V_{0}, V_{1}, f\right)$. All the paths considered depend continuously on the point $\left(V_{0}, V_{1}, f\right)$ that we started with, so we have defined a homotopy $\alpha \beta \simeq 1$. 
Theorem 8.9 The map $q: I_{r}\left(d_{0}, d_{1}\right)_{E} \rightarrow \operatorname{Int}_{r}\left(d_{0}, d_{1}\right)$ is an isomorphism.

Proof We first replace $I_{r}\left(d_{0}, d_{1}\right)$ by the homotopy-equivalent space $I_{r}^{\prime}\left(d_{0}, d_{1}\right)$. We write $I_{r}=I_{r}^{\prime}\left(d_{0}, d_{1}\right)$ and $G_{r}=G_{r}\left(d_{0}, d_{1}\right)$ for brevity, and similarly for Int $_{r}$ and $\mathrm{Sub}_{r}$. We first claim that there is a commutative diagram as follows.

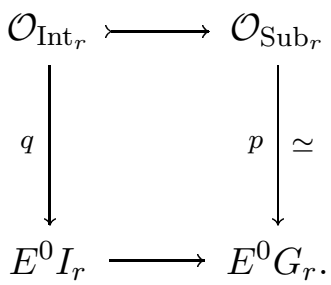

Indeed, the isomorphism

$$
p: \mathcal{O}_{\operatorname{Sub}_{r}\left(d_{0}, d_{1}\right)} \rightarrow E^{0} G_{r}\left(d_{0}, d_{1}\right)
$$

comes from Theorem 8.2, and the map $q$ comes from Definition 8.7. It was proved in Theorem 6.3 that the top horizontal map is a split monomorphism of $\mathcal{O}_{S}$-modules, and it follows that the same is true of the map $q: \mathcal{O}_{\mathrm{Int}_{r}} \rightarrow E^{0} I_{r}$.

We now specialise to the case where $E$ is $H\left[u, u^{-1}\right]$, the two-periodic version of the integer Eilenberg-MacLane spectrum. We then have $E^{0} X=\prod_{k} H^{2 k} X$ for all spaces $X$. This splits each of the rings on the bottom row of our diagram as a product of homogeneous pieces, and it is not hard to check that there is a unique compatible way to split the rings on the top row. We know that $q$ is a split monomorphism; if we can show that the source and target have the same Poincaré series, it will follow that $q$ is an isomorphism. If $r=\min \left(d_{0}, d_{1}\right)$ then $\mathrm{Int}_{r}=\mathrm{Sub}_{r}$ so the claim is certainly true. To work downwards from here by induction, it will suffice to show that

$$
P S\left(H^{*} I_{r+1}\right)-P S\left(H^{*} I_{r}\right)=P S\left(\mathcal{O}_{\text {Int }_{r+1}}\right)-P S\left(\mathcal{O}_{\text {Int }_{r}}\right)
$$

for all $r$.

To evaluate the left hand side, we consider the space

$$
I_{r} \backslash I_{r+1}=\left\{\left(V_{0}, V_{1}\right) \in G_{d_{0}}\left(\mathbb{C}^{\infty}\right) \times G_{d_{1}}\left(\mathbb{C}^{\infty}\right) \mid \operatorname{dim}\left(V_{0} \cap V_{1}\right)=k\right\} .
$$

Let $G_{r}^{\prime}$ be the space of triples $\left(V, V_{0}^{\prime}, V_{1}^{\prime}\right)$ of mutually orthogonal subspaces of $\mathbb{C}^{\infty}$ such that $\operatorname{dim}(V)=r$ and $\operatorname{dim}\left(V_{i}\right)=d_{i}-r$. This is well-known to be a model of $B U(r) \times B U\left(d_{0}-r\right) \times B U\left(d_{1}-r\right)$ and thus homotopyequivalent to $G_{r}$; the argument uses frames much as in the proof of Theorem 8.2. Let $W$ be the bundle over $G_{r}^{\prime}$ whose fibre over $\left(V, V_{0}^{\prime}, V_{1}^{\prime}\right)$ is $\operatorname{Hom}\left(V_{1}^{\prime}, V_{0}^{\prime}\right)$. If $\alpha \in \operatorname{Hom}\left(V_{0}^{\prime}, V_{1}^{\prime}\right)$ and we put $V_{0}=V \oplus V_{1}^{\prime}$ and $V_{1}=V \oplus \operatorname{graph}(\alpha)$ then 
$V_{0} \cap V_{1}=V$ and so $\left(V_{0}, V_{1}\right) \in I_{r}$. It is not hard to see that this construction gives a homeomorphism of the total space of $W$ with $I_{r} \backslash I_{r+1}$. This in turn gives a homeomorphism of the Thom space $\left(G_{r}^{\prime}\right)^{W}$ with the quotient space $I_{r} / I_{r+1}$. By induction we may assume that $H^{*} I_{r+1}$ is concentrated in even degrees, and it is clear from the Thom isomorphism theorem that the same is true of $\widetilde{H}^{*}\left(G_{r}^{\prime}\right)^{W}$. This implies that $H^{*} I_{r}$ is in even degrees and that $P S\left(H^{*} I_{r}\right)-$ $P S\left(H^{*} I_{r+1}\right)=P S\left(\widetilde{H}^{*}\left(G_{r}^{\prime}\right)^{W}\right)$. As $W$ has dimension $\left(d_{0}-r\right)\left(d_{1}-r\right)$, we see that $P S\left(\widetilde{H}^{*}\left(G_{r}^{\prime}\right)^{W}\right)=t^{2\left(d_{0}-r\right)\left(d_{1}-r\right)} P S\left(H^{*} G_{r}^{\prime}\right)$. We also know that $H^{*} G_{r}^{\prime} \simeq$ $\mathcal{O}_{\mathrm{Sub}_{r}}$. The conclusion is that

$$
P S\left(H^{*} I_{r}\right)-P S\left(H^{*} I_{r+1}\right)=t^{2\left(d_{0}-r\right)\left(d_{1}-r\right)} P S\left(\mathcal{O}_{\mathrm{Sub}_{r}}\right) .
$$

We next evaluate $P S\left(\mathcal{O}_{\text {Int }_{r+1}}\right)-P S\left(\mathcal{O}_{\text {Int }_{r}}\right)$. Put

$$
R_{r}^{*}=\mathbb{Z} \llbracket c_{01}, \ldots, c_{0, d_{0}-r}, c_{11}, \ldots, c_{1, d_{1}} \rrbracket .
$$

We know from Theorem 6.3 that $\mathcal{O}_{\text {Int }_{r}}$ is freely generated over $R_{r}^{*}$ by the monomials $\prod_{i=1}^{r} c_{0, d_{0}-r+i}^{\alpha_{i}}$ for which $\sum_{i=1}^{r} \alpha_{i} \leq d_{1}-r$. It follows that the monomials $\prod_{i=0}^{r} c_{0, d_{0}-r+i}^{\alpha_{i}}$ for which $\sum_{1}^{r} \alpha_{i} \leq d_{1}-r$ form a basis for $\mathcal{O}_{\text {Int }_{r}}$ over $R_{r+1}^{*}$. Similarly, those for which $\sum_{i=0}^{r} \alpha_{i}<d_{1}-r$ form a basis for $\mathcal{O}_{\text {Int }_{r+1}}$ over $R_{r+1}^{*}$. Thus, if we let $M^{*}$ be the module generated over $R_{r+1}^{*}$ by the monomials with $\sum_{1}^{r} \alpha_{i} \leq d_{1}-r \leq \sum_{0}^{r} \alpha_{i}$, we find that $P S\left(\mathcal{O}_{\text {Int }_{r+1}}\right)-P S\left(\mathcal{O}_{\text {Int }_{r}}\right)=P S\left(M^{*}\right)$.

It is not hard to check that the monomials for which $\sum_{0}^{r} \alpha_{i}=d_{1}-r$ form a basis for $M^{*}$ over $R_{r}^{*}$. Next, let $N^{*}$ be generated over $\mathbb{Z}$ by the monomials $\prod_{i=0}^{r} c_{i}^{\alpha_{i}}$ for which $\sum_{0}^{r} \alpha_{i}=d_{1}-r$; note that this involves the variables $1=c_{0}, \ldots, c_{r}$ rather than the variables $c_{d_{0}-r}, \ldots, c_{d_{0}}$ used in $M^{*}$. Because $\operatorname{deg}\left(c_{d_{0}-r+i}\right)=$ $\operatorname{deg}\left(c_{i}\right)+2\left(d_{0}-r\right)$ we have

$$
\operatorname{deg}\left(\prod_{i} c_{d_{0}-r+i}^{\alpha_{i}}\right)=\operatorname{deg}\left(\prod_{i} c_{i}^{\alpha_{i}}\right)+2\left(d_{0}-r\right) \sum_{i} \alpha_{i} .
$$

Using this, we see that $P S\left(M^{*}\right)=t^{2\left(d_{0}-r\right)\left(d_{1}-r\right)} P S\left(N^{*}\right) P S\left(R_{r}^{*}\right)$. However, Corollary 6.11 essentially says that $\mathcal{O}_{\mathrm{Sub}_{r}} \simeq R_{r}^{*} \otimes N^{*}$ as graded Abelian groups, so $P S\left(N^{*}\right) P S\left(R_{r}^{*}\right)=P S\left(\mathcal{O}_{\operatorname{Sub}_{r}}\right)$, so

$$
\begin{aligned}
P S\left(\mathcal{O}_{\mathrm{Int}_{r+1}}\right)-P S\left(\mathcal{O}_{\mathrm{Int}_{r}}\right) & =t^{2\left(d_{0}-r\right)\left(d_{1}-r\right)} P S\left(\mathcal{O}_{\mathrm{Sub}_{r}}\right) \\
& =P S\left(H^{*} I_{r}\right)-P S\left(H^{*} I_{r+1}\right) .
\end{aligned}
$$

As explained previously, this implies that $q$ is an isomorphism in the case $E=H\left[u, u^{-1}\right]$. We next consider the case $E=M U\left[u, u^{-1}\right]$. Let $I$ be the kernel of the usual map $M U_{*} \rightarrow \mathbb{Z}$. Because $H^{*} I_{r}$ is free of finite type and concentrated in even degrees, we see that the Atiyah-Hirzebruch spectral sequence collapses and that the associated graded ring $\operatorname{gr}_{I} M U^{*} I_{r}$ is isomorphic 
to $\operatorname{gr}_{I}\left(M U^{*}\right) \widehat{\otimes} H^{*} I_{r}$. Using this it is not hard to check that $q$ is an isomorphism in the case $E=M U\left[u, u^{-1}\right]$ also. Finally, given an arbitrary even periodic ring spectrum $E$ we can choose a complex orientation in $\widetilde{E}^{0} \mathbb{C} P^{\infty}$ and thus a ring map $M U\left[u, u^{-1}\right] \rightarrow E$. Using this, we deduce that $q$ is an isomorphism for all E.

Corollary 8.10 Let $V_{0}$ and $V_{1}$ be bundles of dimensions $d_{0}$ and $d_{1}$ over a space $X$. Then there is a spectral sequence

$$
\operatorname{Tor}_{E^{*} B U\left(d_{0}\right) \times B U\left(d_{1}\right)}^{* *}\left(E^{*} X, E^{*} I_{r}\left(d_{0}, d_{1}\right)\right) \Longrightarrow E^{*} I_{r}\left(V_{0}, V_{1}\right),
$$

whose edge map in degree zero is the map

$$
q^{*}: \mathcal{O}_{\operatorname{Int}_{r}\left(D\left(V_{0}\right), D\left(V_{1}\right)\right)} \rightarrow E^{0} I_{r}\left(V_{0}, V_{1}\right) .
$$

The spectral sequence collapses in the semiuniversal and universal cases. (We do not address the question of convergence in the general case.)

Proof This is another Eilenberg-Moore spectral sequence.

\section{The schemes $P_{k} D$}

Let $D$ be a divisor of degree $d$ on $\mathbb{G}$ over $S$, with equation

$$
f(t)=f_{D}(t)=\sum_{i=0}^{d} c_{i} x^{d-i} \in \mathcal{O}_{S}[t],
$$

say. In this section we assemble some useful facts about the scheme $P_{k} D$. This is a closed subscheme of $\mathbb{G}^{k}$, so $\mathcal{O}_{P_{k} D}=\mathcal{O}_{S} \llbracket x_{0}, \ldots, x_{k-1} \rrbracket / J_{k}$ for some ideal $J_{k}$; our main task will be to find systems of generators for $J_{k}$. We put $p_{i}(t)=\prod_{j<i}\left(t-x_{j}\right)$, and we let $q_{i}(t)$ and $r_{i}(t)$ be the quotient and remainder when $f(t)$ is divided by $p_{i}(t)$. Thus $f(t)=q_{i}(t) p_{i}(t)+r_{i}(t)$ and $r_{i}(t)$ has the form $\sum_{j=0}^{i-1} a_{i j} t^{j}$ for some $a_{i 0}, \ldots, a_{i, i-1} \in \mathcal{O}_{S}$. From the definitions it is clear that $J_{k}$ is the smallest ideal modulo which $f(t)$ becomes divisible by $p_{k}(t)$, or in other words the smallest ideal modulo which $r_{k}(t)=0$, so $J_{k}$ is generated by $a_{k 0}, \ldots, a_{k, k-1}$. Now put $b_{i}=a_{i+1, i}$ for $0 \leq i<k$; we will show that these elements also generate $J_{k}$.

Lemma 9.1 We have $b_{i}=q_{i}\left(x_{i}\right)$ and $r_{i+1}=b_{i} p_{i}+r_{i}$ for all $i$. 
Proof The polynomial $q_{i}(t)-q_{i}\left(x_{i}\right)$ is evidently divisible by $t-x_{i}$, say $q_{i}(t)-$ $q_{i}\left(x_{i}\right)=\left(t-x_{i}\right) q_{i+1}^{\prime}(t)$. If we put $r_{i+1}^{\prime}(t)=q_{i}\left(x_{i}\right) p_{i}(t)+r_{i}(t)$ we find that $r_{i+1}^{\prime}$ is a polynomial of degree at most $i$ and that $f(t)=q_{i+1}^{\prime}(t) p_{i+1}(t)+r_{i+1}^{\prime}(t)$, so we must have $q_{i+1}=q_{i+1}^{\prime}$ and $r_{i+1}=r_{i+1}^{\prime}$. Thus $b_{i}$ is the coefficient of $t^{i}$ in $r_{i+1}^{\prime}(t)$. As $r_{i}$ has degree less than $i$ and $p_{i}$ is monic of degree $i$ we deduce that $b_{i}=q_{i}\left(x_{i}\right)$.

Corollary 9.2 The ideal $J_{k}$ is generated by $b_{0}, \ldots, b_{k-1}$.

Proof Put $J_{k}^{\prime}=\left(b_{0}, \ldots, b_{k-1}\right)$. If we work modulo $J_{k}^{\prime}$ then it is immediate from the lemma that $r_{k}=r_{k-1}=\ldots=r_{0}=0$; this shows that $J_{k} \subseteq J_{k}^{\prime}$. Conversely, if we work modulo $J_{k}$ then $f$ is divisible by $p_{k}$ and hence by $p_{i}$ for all $i \leq k$, so $r_{0}=\ldots=r_{k}=0$. It follows from the lemma that $b_{i} p_{i}=0$ for all $i$, and $p_{i}$ is monic so $b_{i}=0$. Thus $J_{k}^{\prime} \subseteq J_{k}$.

We now give a determinantal formula for the relators $b_{j}$. Consider the Vandermonde determinant

$$
v_{k}:=\operatorname{det}\left(x_{i}^{j}\right)_{0 \leq i, j<k}=\prod_{0 \leq i<j<k}\left(x_{j}-x_{i}\right) .
$$

We also define a matrix $B_{k}$ by

$$
\left(B_{k}\right)_{i j}= \begin{cases}x_{i}^{j} & \text { if } 0 \leq j<k-1 \\ f\left(x_{i}\right) & \text { if } j=k-1\end{cases}
$$

Proposition 9.3 We have $b_{j}=\operatorname{det}\left(B_{j}\right) / v_{j}$ for all $j$. (More precisely, we have $v_{j} b_{j}=\operatorname{det}\left(B_{j}\right) \in \mathcal{O}_{S} \llbracket x_{0}, \ldots, x_{j-1} \rrbracket$, and $v_{j}$ is not a zero-divisor in this ring.)

Proof Define $\alpha: \mathcal{O}_{S}{ }^{j} \rightarrow \mathcal{O}_{S} \llbracket t \rrbracket / p_{j}(t)$ by

$\alpha\left(u_{0}, \ldots, u_{j-2}, w\right)=\sum_{i} u_{i} t^{i}+w f(t) \quad\left(\bmod p_{j}\right)=\sum_{i} u_{i} t^{i}+w r_{j}(t) \quad\left(\bmod p_{j}\right)$.

Next, define $\beta: \mathcal{O}_{S} \llbracket t \rrbracket / p_{j}(t) \rightarrow \mathcal{O}_{S}^{j}$ by $\beta(g)=\left(g\left(x_{0}\right), \ldots, g\left(x_{j-1}\right)\right)$. We identify $\mathcal{O}_{S} \llbracket t \rrbracket / p_{j}(t)$ with $\mathcal{O}_{S}{ }^{j}$ using the basis $\left\{t^{i} \mid 0 \leq i<j\right\}$. It is easy to see that $\operatorname{det}(\beta)=v_{j}$ and $\operatorname{det}(\beta \alpha)=\operatorname{det}\left(B_{j}\right)$. Moreover, the matrix of $\alpha$ has the form $\left(\begin{array}{c|c}I & * \\ \hline 0 & b_{j}\end{array}\right)$ so $\operatorname{det}(\alpha)=b_{j}$. It follows immediately that $v_{j} b_{j}=\operatorname{det}\left(B_{j}\right)$. It is easy to see that none of the polynomials $x_{j}-x_{i}$ (where $i<j$ ) are zero-divisors, so $v_{j}$ is not a zero-divisor. 
We next need to relate the schemes $P_{k} D$ to the exterior powers $\lambda^{k} \mathcal{O}_{D}$.

Lemma 9.4 The ideal $J_{k}$ maps to zero under the natural projection $\mathcal{O}_{D^{k}}=$ $\mathcal{O}_{D}^{\otimes k} \rightarrow \lambda^{k} \mathcal{O}_{D}$

Proof It is enough to prove the corresponding result in the universal case, where $D$ is the tautological divisor over $\operatorname{Div}_{d}^{+}$. As the map $\mathbb{G}^{d} \rightarrow \mathbb{G}^{d} / \Sigma_{d}=$ $\operatorname{Div}_{d}^{+}$is faithfully flat, it is enough to prove the result after pulling back along this map. In other words, we need only consider the divisor over the ring $R:=\mathcal{O}_{\mathbb{G}^{d}}=\mathcal{O}_{S} \llbracket y_{i} \mid i<d \rrbracket$ with equation $f(t)=\prod_{i}\left(t-y_{i}\right)$. Let $w$ be the discriminant of this polynomial, so $w=\prod_{i \neq j}\left(y_{i}-y_{j}\right) \in R$. Put $N=$ $\{0, \ldots, d-1\}$, and let $F(N, R)$ denote the ring of functions from $N$ to $R$, with pointwise operations. We can define $\phi: \mathcal{O}_{D} \rightarrow F(N, R)$ by $\phi(g)(i)=$ $g\left(y_{i}\right)$, and the Chinese Remainder Theorem tells us that the resulting map $w^{-1} \mathcal{O}_{D} \rightarrow F\left(N, w^{-1} R\right)$ is an isomorphism, and it follows that $w^{-1} \mathcal{O}_{D^{k}}=$ $F\left(N^{k}, w^{-1} R\right)$. We also have $\mathcal{O}_{D^{k}}=R \llbracket x_{j} \mid j<k \rrbracket /\left(f_{D}\left(x_{j}\right) \mid j<k\right)$; the element $x_{j}$ corresponds to the function $\underline{n} \mapsto y_{n_{j}}$.

Now put

$$
N_{k}=\left\{\left(n_{0}, \ldots, n_{k-1}\right) \in N^{k} \mid n_{i} \neq n_{j} \text { when } i \neq j\right\},
$$

and $N_{k}^{c}=N^{k} \backslash N_{k}$. Let $r(t)$ be the remainder when the polynomial $f_{D}(t):=$ $\prod_{i<d}\left(t-y_{i}\right)$ is divided by $f_{D^{\prime}}(t):=\prod_{j<k}\left(t-x_{j}\right)$. This corresponds to the function $\underline{n} \mapsto r_{\underline{n}}(t)$, where $r_{\underline{n}}(t)$ is the remainder of $f_{D}(t)$ modulo $\prod_{j<k}(t-$ $\left.y_{n_{j}}\right)$. As the discriminant is invertible in $w^{-1} R$ we see that $r_{\underline{n}}(t)=0$ iff $\underline{n} \in N_{k}$, and otherwise some coefficient of $r_{\underline{n}}(t)$ is invertible. Using this, we deduce that $w^{-1} \mathcal{O}_{P_{k} D}=F\left(N_{k}, w^{-1} R\right)$ and $w^{-1} J_{k}=F\left(N_{k}^{c}, w^{-1} R\right)$. If we let $\left\{e_{0}, \ldots, e_{k-1}\right\}$ be the evident basis of $F(N, R)$ over $R$, this means that $w^{-1} J_{k}$ is spanned over $w^{-1} R$ by the elements $e_{n_{0}} \otimes \ldots \otimes e_{n_{k-1}}$ for which $n_{i}=n_{j}$ for some $i \neq j$, and these elements satisfy $e_{n_{0}} \wedge \ldots \wedge e_{n_{k-1}}=0$ so the map $w^{-1} J_{k} \rightarrow w^{-1} \lambda^{k} \mathcal{O}_{D}$ is zero. As $w$ is not a zero-divisor we deduce that the map $J_{k} \rightarrow \lambda^{k} \mathcal{O}_{D}$ is zero, as claimed.

Next note that the symmetric group $\Sigma_{k}$ acts on $D^{k}$ and $P_{k} D$ and thus on the

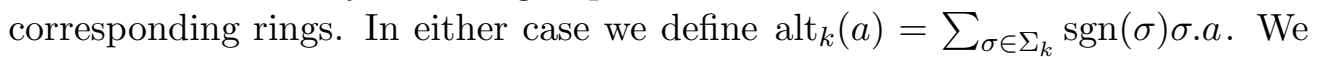
also let $\mu_{k}: \mathcal{O}_{D}^{\otimes k} \rightarrow \lambda^{k} \mathcal{O}_{D}$ be the usual projection, or equivalently the restriction of the product map $\mu_{k}:\left(\lambda^{*} \mathcal{O}_{D}\right)^{\otimes k} \rightarrow \lambda^{*} \mathcal{O}_{D}$. Dually, we let $\psi_{k}: \lambda^{k} \mathcal{O}_{D} \rightarrow$ $\mathcal{O}_{D}^{\otimes k}$ be the component of the coproduct map $\psi_{k}: \lambda^{*} \mathcal{O}_{D} \rightarrow\left(\lambda^{*} \mathcal{O}_{D}\right)^{\otimes k}$. We also let $p^{*}: \mathcal{O}_{D}^{\otimes k}=\mathcal{O}_{D^{k}} \rightarrow \mathcal{O}_{P_{k} D}$ denote the usual projection, corresponding to the closed inclusion $P_{k} D \rightarrow D^{k}$. 
Proposition 9.5 There is a natural commutative diagram as follows.

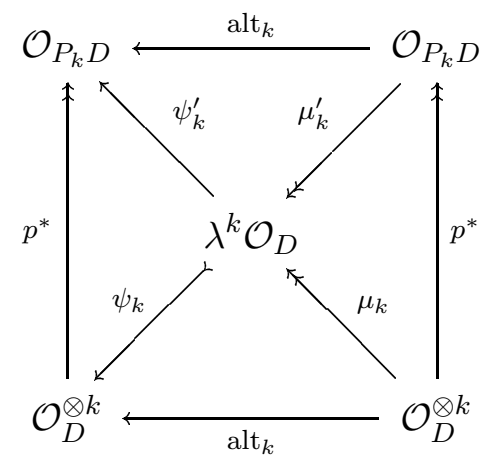

Proof The main point to check is that $\psi_{k} \mu_{k}=\operatorname{alt}_{k}: \mathcal{O}_{D}^{\otimes k} \rightarrow \mathcal{O}_{D}^{\otimes k}$. Consider an element $a=a_{0} \otimes \ldots \otimes a_{k-1} \in \mathcal{O}_{D}^{\otimes k}$. Let $a_{i}^{j}$ denote the element $1^{\otimes j} \otimes a_{i} \otimes$ $1^{\otimes k-j-1} \in \mathcal{O}_{D}^{\otimes k}$, so that $\psi_{k}\left(a_{i}\right)=\sum_{j=0}^{k-1} a_{i}^{j}$ and $\psi_{k} \mu_{k}(a)=\prod_{i} \sum_{j} a_{i}^{j}$. We are interested in the component of this in $\mathcal{O}_{D}^{\otimes k} \subset\left(\lambda^{*} \mathcal{O}_{D}\right)^{\otimes k}$, which is easily seen to be $\sum_{\sigma} \prod_{i} a_{i}^{\sigma(i)}$. Moreover, one checks that

$$
\prod_{i} a_{i}^{\sigma(i)}=\operatorname{sgn}(\sigma) a_{\sigma^{-1}(0)} \otimes \ldots \otimes a_{\sigma^{-1}(k-1)}=\operatorname{sgn}(\sigma) \sigma . a,
$$

so the relevant component of $\psi_{k} \mu_{k}(a)$ is $\sum_{\sigma} \operatorname{sgn}(\sigma) \sigma \cdot a=\operatorname{alt}_{k}(a)$, as claimed.

Let $A$ be the set of multiindices $\alpha=\left(\alpha_{0}, \ldots, \alpha_{k-1}\right)$ with $0 \leq \alpha_{i}<d$ for all $i$, and let $A_{0}$ be the subset of those for which $\alpha_{0}>\ldots>\alpha_{k-1}$. Put $x^{\alpha}:=x^{\alpha_{0}} \otimes \ldots \otimes x^{\alpha_{k-1}} \in \mathcal{O}_{D}^{\otimes k}$. Then $\left\{x^{\alpha} \mid \alpha \in A\right\}$ is a basis for $\mathcal{O}_{D}^{\otimes k}$, and $\left\{\mu_{k}\left(x^{\alpha}\right) \mid \alpha \in A_{0}\right\}$ is a basis for $\lambda^{k} \mathcal{O}_{D}$. Moreover, if $\alpha \in A_{0}$ and we write $\psi_{k} \mu_{k}\left(x^{\alpha}\right)=\operatorname{alt}_{k}\left(x^{\alpha}\right)=\sum_{\beta \in A} c_{\alpha \beta} x^{\beta}$ we see that $c_{\alpha \alpha}=1$ and $c_{\alpha \beta}=0$ if $\beta \in A_{0}$ and $\beta \neq \alpha$. It follows that $\mu_{k}$ is surjective and $\psi_{k}$ is a split injection of $\mathcal{O}_{S}$-modules, as indicated in the diagram.

Lemma 9.4 tells us that $\mu_{k}$ factor as $\mu_{k}^{\prime} p^{*}$ for some $\mu_{k}^{\prime}: \mathcal{O}_{P_{k} D} \rightarrow \lambda^{k} \mathcal{O}_{D}$, and a diagram chase shows that $\mu_{k}^{\prime}$ is surjective. This gives the right hand triangle of the diagram. We simply define $\psi_{k}^{\prime}=p^{*} \psi_{k}$ to get the left hand triangle. As $p^{*}$ is $\Sigma_{k}$-equivariant we have

$$
\operatorname{alt}_{k} p^{*}=p^{*} \operatorname{alt}_{k}=p^{*} \psi_{k} \mu_{k}=\psi_{k}^{\prime} \mu_{k}^{\prime} p^{*} .
$$

As $p^{*}$ is surjective, this proves that $\psi_{k}^{\prime} \mu_{k}^{\prime}=\operatorname{alt}_{k}$, so the top triangle commutes.

We next study certain orbit schemes for actions of $\Sigma_{k}$. Recall that $\mathcal{O}_{\mathbb{G}^{k}}=$ $\mathcal{O}_{S} \llbracket x_{i} \mid i<k \rrbracket$ has a topological basis consisting of monomials in the variables 
$x_{i}$. This basis is permuted by $\Sigma_{k}$, and the sums of the orbits form a topological basis for the invariant subring $\mathcal{O}_{\mathbb{G}^{k}}^{\Sigma_{k}}=\mathcal{O}_{\mathbb{G}^{k} / \Sigma_{k}}=\mathcal{O}_{\operatorname{Div}_{k}^{+}}$. It is clear from this analysis that our quotient construction commutes with base change, in other words $\left(S^{\prime} \times{ }_{S} \mathbb{G}^{k}\right) / \Sigma_{k}=S^{\prime} \times{ }_{S}\left(\mathbb{G}^{k} / \Sigma_{k}\right)$ for any scheme $S^{\prime}$ over $S$. Similarly, the set $\left\{x^{\alpha} \mid \alpha_{i}<d\right.$ for all $\left.i\right\}$ is a basis for $\mathcal{O}_{D^{k}}$ that is permuted by $\Sigma_{k}$, so the orbit sums give a basis for $\mathcal{O}_{D^{k}}^{\Sigma_{k}}$ and we have a quotient scheme $D^{k} / \Sigma_{k}=$ $\operatorname{spf}\left(\mathcal{O}_{D^{k}}^{\Sigma_{k}}\right)$ whose formation commutes with base change. By comparing our bases we see that the projection $\mathcal{O}_{\mathbb{G}} \rightarrow \mathcal{O}_{D}=\mathcal{O}_{\mathbb{G}} / f_{D}$ induces a surjective map $\mathcal{O}_{\mathbb{G}^{k} / \Sigma_{k}} \rightarrow \mathcal{O}_{D^{k} / \Sigma_{k}}$. In other words, we have a commutative square of schemes as shown, in which $j$ and $j^{\prime}$ are closed inclusions, and $q_{2}$ is a faithfully flat map of degree $d$ !

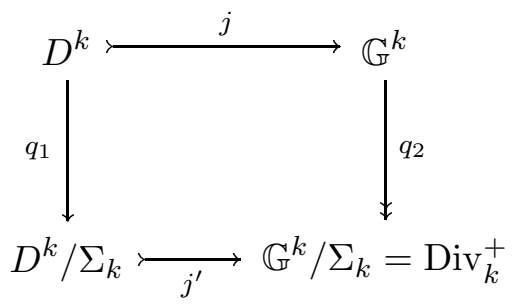

One might hope to show that $P_{k}(D) / \Sigma_{k}=\operatorname{Sub}_{k}(D)$ in a similar sense, but this is not quite correct. For example if $D=3[0]$ ( $s o f_{D}(t)=t^{3}$ ) and $k=2$ then $\mathcal{O}_{P_{2} D}=\mathcal{O}_{S} \llbracket x, y \rrbracket /\left(x^{3}, x^{2}+x y+y^{2}\right)$. If we define a basis of this ring by

$$
\left\{e_{0}, \ldots, e_{5}\right\}=\left\{1, x, y, x^{2},-x^{2}-x y, x^{2} y\right\},
$$

we find that the generator of $\Sigma_{2}$ has the effect

$$
e_{0} \leftrightarrow e_{0}, e_{1} \leftrightarrow e_{2}, e_{3} \leftrightarrow e_{4}, e_{5} \leftrightarrow-e_{5}
$$

If $\mathcal{O}_{S}$ has no 2 -torsion we find that $\mathcal{O}_{P_{2} D}^{\Sigma_{2}}$ is spanned by $\{1, x+y, x y\}$ and thus is equal to $\mathcal{O}_{\mathrm{Sub}_{2}(D)}$. However, if $2=0$ in $\mathcal{O}_{S}$ we have an additional generator $x^{2} y$, so $\mathcal{O}_{P_{2} D}^{\Sigma_{2}}$ is strictly larger than $\mathcal{O}_{\mathrm{Sub}_{2}(D)}$. This example also shows that the formation of $\mathcal{O}_{P_{2} D}^{\Sigma_{2}}$ is not compatible with base change.

The following proposition provides a substitute for the hope described above.

Proposition 9.6 There is a commutative diagram as follows, in which $i$, $i^{\prime}, j$ and $j^{\prime}$ are closed inclusions, and $q_{0}$ and $q_{2}$ are faithfully flat of degree $k$ !. Moreover, the outer rectangle is a pullback, and if $J_{k}:=\operatorname{ker}\left(i^{*}\right)$ then 


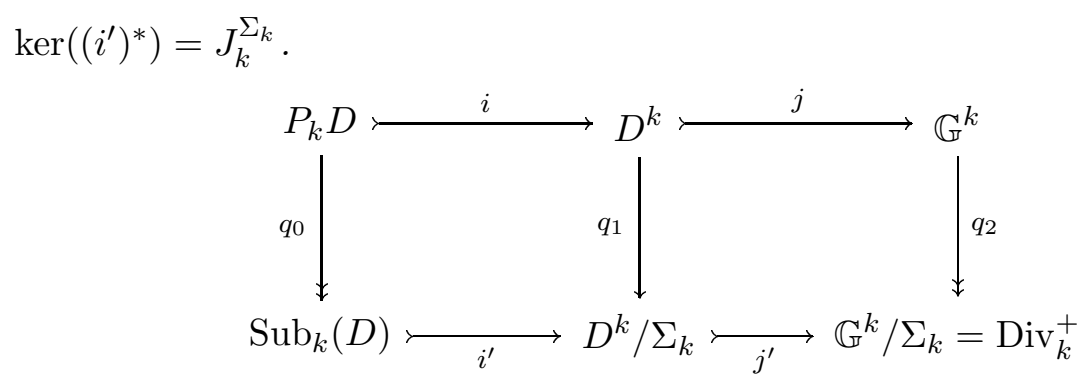

Proof We have already produced the right hand square. The map $i$ is just the obvious inclusion. The map $q_{0}$ sends $\left(a_{0}, \ldots, a_{k-1}\right) \in P_{k} D$ to $\left[a_{0}\right]+\ldots+$ $\left[a_{k-1}\right] \in \operatorname{Sub}_{k}(D)$; it was observed in the proof of Lemma 6.9 that this makes $\mathcal{O}_{P_{k} D}$ into a free module of rank $k$ ! over $\mathcal{O}_{\operatorname{Sub}_{k}(D)}$, so $q_{0}$ is faithfully flat of degree $k !$.

The points of $\operatorname{Sub}_{k}(D)$ are the divisors of degree $k$ contained in $D$, so $\operatorname{Sub}_{k}(D)$ is a closed subscheme of $\operatorname{Div}_{k}^{+}$; we write $m^{\prime}: \operatorname{Sub}_{k}(D) \rightarrow \operatorname{Div}_{k}^{+}$for the inclusion, and note that $m^{\prime} q_{0}=q_{2} j i$. As $q_{0}$ is faithfully flat and $m^{\prime} q_{0}$ factors through $D^{k} / \Sigma_{k}$ we see that $m^{\prime}$ factors through $D^{k} / \Sigma_{k}$, so there is a unique map $i^{\prime}: \operatorname{Sub}_{k}(D) \rightarrow D^{k} / \Sigma_{k}$ such that $m^{\prime}=j^{\prime} i^{\prime}$. As $m^{\prime}$ is a closed inclusion, the same is true of $i^{\prime}$. A point of the pullback of $m$ and $q_{2}$ is a list $a=\left(a_{0}, \ldots, a_{k-1}\right)$ of points of $\mathbb{G}$ such that the divisor $q_{2}(a)=\sum_{r}\left[a_{r}\right]$ lies in $\operatorname{Sub}_{k}(D)$, and thus satisfies $\sum_{r}\left[a_{r}\right] \leq D$. It follows from the definitions that this pullback is just $P_{k} D$ as claimed.

As $q_{0}$ is faithfully flat we have $\operatorname{ker}\left(\left(i^{\prime}\right)^{*}\right)=\operatorname{ker}\left(q_{0}^{*}\left(i^{\prime}\right)^{*}\right)=\operatorname{ker}\left(i^{*} q_{1}^{*}\right)$. By construction, $q_{1}^{*}$ is just the inclusion of the $\Sigma_{k}$-invariants in $\mathcal{O}_{D^{k}}$, so $\operatorname{ker}\left(i^{*} q_{1}^{*}\right)=$ $\operatorname{ker}\left(i^{*}\right)^{\Sigma_{k}}=J_{k}^{\Sigma_{k}}$ as claimed.

Corollary 9.7 $\lambda^{k} \mathcal{O}_{D}$ is naturally a module over $\mathcal{O}_{\operatorname{Sub}_{k}(D)}$.

Proof We can certainly regard $\mathcal{O}_{D^{k}}$ as a module over the subring $\mathcal{O}_{D^{k} / \Sigma_{k}}=$ $\mathcal{O}_{D^{k}}^{\Sigma_{k}}$, and the map alt ${ }_{k}: \mathcal{O}_{D^{k}} \rightarrow \mathcal{O}_{D^{k}}$ respects this structure. This makes $\lambda^{k} \mathcal{O}_{D}=$ image $\left(\right.$ alt $\left._{k}\right)$ into a module over $\mathcal{O}_{D^{k}}^{\Sigma_{k}}$. If $a \in J_{k}^{\Sigma_{k}}$ and $b \in \mathcal{O}_{D^{k}}$ then $a \operatorname{alt}_{k}(b)=\operatorname{alt}_{k}(a b)$ but $a b \in J_{k}$ so $\operatorname{alt}_{k}(a b)=0$. This shows that $\lambda^{k} \mathcal{O}_{D}$ is annihilated by $J_{k}^{\Sigma_{k}}$, so it is a module over $\mathcal{O}_{D^{k}}^{\Sigma_{k}} / J_{k}^{\Sigma_{k}}=\mathcal{O}_{\operatorname{Sub}_{k}(D)}$, as claimed.

We next identify $\lambda^{k} \mathcal{O}_{D}$ as a module over $\mathcal{O}_{\operatorname{Sub}_{k}(D)}$. Let $D^{\prime}$ be the tautological divisor of degree $k$ over $\operatorname{Sub}_{k}(D)$. Then $\mathcal{O}_{D^{\prime}}$ is naturally a quotient of the ring $\mathcal{O}_{D} \otimes_{S} \mathcal{O}_{\operatorname{Sub}_{k}(D)}$, which contains the subring $\mathcal{O}_{D}=\mathcal{O}_{D} \otimes 1$. This gives us a map $\mathcal{O}_{D} \rightarrow \mathcal{O}_{D^{\prime}}$, which extends to give a map $\phi: \lambda_{S}^{k} \mathcal{O}_{D} \rightarrow \lambda_{\operatorname{Sub}_{k}(D)}^{k} \mathcal{O}_{D^{\prime}}$. 
Proposition 9.8 The map $\phi: \lambda_{S}^{k} \mathcal{O}_{D} \rightarrow \lambda_{\operatorname{Sub}_{k}(D)}^{k} \mathcal{O}_{D^{\prime}}$ is an isomorphism of free rank-one modules over $\mathcal{O}_{\mathrm{Sub}_{k}(D)}$.

Proof Put $T=\operatorname{Sub}_{k}(D)$ for brevity. Note that

$$
\begin{aligned}
\mathcal{O}_{D} & =\mathcal{O}_{S}\left\{x^{i} \mid i<d\right\} \\
\lambda_{S}^{k} \mathcal{O}_{D} & =\mathcal{O}_{S}\left\{x^{i_{0}} \wedge \ldots \wedge x^{i_{k-1}} \mid d>i_{0}>\ldots>i_{k-1}\right\} \\
\mathcal{O}_{D^{\prime}} & =\mathcal{O}_{T}\left\{x^{i} \mid i<k\right\} \\
\lambda_{T}^{k} \mathcal{O}_{D^{\prime}} & =\mathcal{O}_{T}\left\{x^{k-1} \wedge \ldots \wedge x^{0}\right\} .
\end{aligned}
$$

In particular, we see that $\lambda_{S}^{k} \mathcal{O}_{D}$ is free of rank $K:=\left(\begin{array}{c}d \\ k\end{array}\right)$ over $\mathcal{O}_{S}$, and $\lambda_{T}^{k} \mathcal{O}_{D^{\prime}}$ is free of rank one over $\mathcal{O}_{T}$. We also know from Lemma 6.9 that $\mathcal{O}_{T}$ is free of rank $K$ over $\mathcal{O}_{S}$, so $\lambda_{T}^{k} \mathcal{O}_{D^{\prime}}$ is also free of rank $K$ over $\mathcal{O}_{S}$.

Suppose for the moment that $\phi$ is a homomorphism of $\mathcal{O}_{T}$-modules. It is clear that

$$
\phi\left(x^{k-1} \wedge \ldots \wedge x^{0}\right)=x^{k-1} \wedge \ldots \wedge x^{0},
$$

and this element generates $\lambda_{T}^{k} \mathcal{O}_{D^{\prime}}$, so $\phi$ is surjective. As the source and target are free of the same finite rank over $\mathcal{O}_{S}$, we deduce that $\phi$ is an isomorphism as claimed.

We still need to prove that $\phi$ is linear over $\mathcal{O}_{T}$. By the argument of Lemma 9.4 we reduce to the case where $D$ is the divisor with equation $\prod_{i=0}^{d-1}\left(t-y_{i}\right)$ defined over the ring

$$
R:=\mathcal{O}_{\mathbb{G}^{d}}=\mathcal{O}_{S} \llbracket y_{0}, \ldots, y_{d-1} \rrbracket,
$$

and we can invert the discriminant $w=\prod_{i \neq j}\left(y_{i}-y_{j}\right)$. We reuse the notation in the proof of that lemma, so $w^{-1} \mathcal{O}_{D}=F\left(N, w^{-1} R\right)$ and $w^{-1} \mathcal{O}_{D^{k}}=$ $F\left(N^{k}, w^{-1} R\right)$ and $w^{-1} \mathcal{O}_{P_{k} D}=F\left(N_{k}, w^{-1} R\right)$. We see from Proposition 9.6 that $w^{-1} \mathcal{O}_{T}$ is the image of $w^{-1} \mathcal{O}_{D^{k}}^{\Sigma_{k}}$ in $w^{-1} \mathcal{O}_{P_{k} D}$, which is the ring $F\left(N_{k}, w^{-1} R\right)^{\Sigma_{k}}$ of symmetric functions from $N_{k}$ to $w^{-1} R$. If we write $N_{k}^{+}=\left\{\underline{n} \in N^{k} \mid n_{0}>\right.$ $\left.\ldots>n_{k-1}\right\}$ then $N_{k}=\Sigma_{k} \times N_{k}^{+}$as $\Sigma_{k}$-sets so $w^{-1} \mathcal{O}_{P_{k} D}=F\left(N_{k}^{+}, w^{-1} R\right)$. On the other hand, $\mathcal{O}_{T}$ is also a quotient of $R \widehat{\otimes} \mathcal{O}_{\operatorname{Div}_{k}^{+}}$, which is the ring of symmetric power series in $k$ variables over $R$; a symmetric power series $p$ corresponds to the function $\underline{n} \mapsto p\left(y_{\underline{n}}\right):=p\left(y_{n_{0}}, \ldots, y_{n_{k-1}}\right)$.

If $\underline{n} \in N^{k}$ we put $e_{\underline{n}}=e_{n_{0}} \otimes \ldots \otimes e_{n_{k-1}}$, so these elements form a basis for $w^{-1} \mathcal{O}_{D}^{\otimes k}$ over $w^{-1} R$. Similarly, the set $\left\{\mu_{k}\left(e_{\underline{n}}\right) \mid \underline{n} \in N_{k}^{+}\right\}$is a basis for $w^{-1} \lambda^{k} \mathcal{O}_{D}$. Using the previous paragraph we see that $p . \mu_{k}\left(e_{\underline{n}}\right)=p\left(y_{\underline{n}}\right) \mu_{k}\left(e_{\underline{n}}\right)$, which tells us the $\mathcal{O}_{T}$-module structure on $w^{-1} \lambda^{k} \mathcal{O}_{D}$. 
We next analyse $w^{-1} \mathcal{O}_{D^{\prime}}$. This is a quotient of the ring

$$
w^{-1} \mathcal{O}_{T} \otimes \mathcal{O}_{D}=F\left(N_{k}^{+}, w^{-1} \mathcal{O}_{D}\right)=\prod_{\underline{n} \in N_{k}^{+}} w^{-1} R\left\{e_{i} \mid i<d\right\} .
$$

It is not hard to check that the relevant ideal is a product of terms $I_{\underline{n}}$, where $I_{\underline{n}}$ is spanned by the elements $e_{i}$ that do not lie in the list $e_{n_{0}}, \ldots, e_{n_{k-1}}$. Thus

$$
\begin{aligned}
w^{-1} \mathcal{O}_{D^{\prime}} & =\prod_{\underline{n}} w^{-1} R\left\{e_{n_{j}} \mid j<k\right\} \\
w^{-1} \lambda_{T}^{k} \mathcal{O}_{D^{\prime}} & =\prod_{\underline{n}} w^{-1} R . e_{n_{0}} \wedge \ldots \wedge e_{n_{k-1}} .
\end{aligned}
$$

Let $e_{\underline{n}}^{\prime}$ be the element of this module whose $\underline{n}$ 'th component is $e_{n_{0}} \wedge \ldots \wedge e_{n_{k-1}}$, and whose other components are zero. Clearly $\left\{e_{\underline{n}}^{\prime} \mid \underline{n} \in N_{k}^{+}\right\}$is a basis for $w^{-1} \lambda_{T}^{k} \mathcal{O}_{D^{\prime}}$ over $w^{-1} R$. As a symmetric power series $p$ corresponds to the function $\underline{n} \mapsto p\left(y_{\underline{n}}\right)$ and $e_{\underline{n}}^{\prime}$ is concentrated in the $\underline{n}$ 'th factor we have $p . e_{\underline{n}}^{\prime}=p\left(y_{\underline{n}}\right) e_{\underline{n}}^{\prime}$. It is also easy to see that $\phi\left(\mu_{k}\left(e_{\underline{n}}\right)\right)=e_{\underline{n}}^{\prime}$, and it follows that $\phi$ is $\mathcal{O}_{T}$-linear as claimed.

We next give a formula for $\phi$ in terms of suitable bases of $\lambda_{S}^{k} \mathcal{O}_{D}$ and $\lambda_{\mathrm{Sub}_{r}}^{k} \mathcal{O}_{D^{\prime}}$. (This could be used to give an alternative proof that $\phi$ is an isomorphism.)

Proposition 9.9 Suppose we have an element $x^{\beta_{0}} \wedge \ldots \wedge x^{\beta_{k-1}} \in \lambda_{S}^{k} \mathcal{O}_{D}$, where $0 \leq \beta_{0}<\ldots<\beta_{d-k-1}$. Let $\gamma_{0}, \ldots, \gamma_{k-1}$ be the elements of $\{0, \ldots, d-1\} \backslash$ $\left\{\beta_{0}, \ldots, \beta_{d-k-1}\right\}$, listed in increasing order. Then

$$
\phi\left(x^{\beta_{0}} \wedge \ldots \wedge x^{\beta_{k-1}}\right)= \pm x^{0} \wedge \ldots \wedge x^{k-1} \cdot \operatorname{det}\left(c_{k+i-\gamma_{j}}\right)_{0 \leq i, j<d-k},
$$

where the elements $c_{i}$ are the usual parameters of the divisor $D^{\prime}$.

Proof For any increasing sequence $\alpha_{0}<\ldots<\alpha_{n-1}$ we write $x(\alpha)=x^{\alpha_{0}} \wedge$ $\ldots \wedge x^{\alpha_{n-1}}$. We also write $e^{\prime}=x(0,1, \ldots, k-1)$ and $e=x(0,1, \ldots, d-1)$, and we put $T=\operatorname{Sub}_{k}(D)$.

We certainly have $\phi(x(\beta))=b_{\beta} e^{\prime}$ for some $b_{\beta} \in \mathcal{O}_{T}$. To analyse these elements, put $J^{\prime}=\operatorname{ker}\left(\mathcal{O}_{T} \otimes \mathcal{O}_{D} \rightarrow \mathcal{O}_{D^{\prime}}\right.$, which is freely generated over $\mathcal{O}_{T}$ by $\left\{x^{i} f_{D^{\prime}}(x) \mid i<d-k\right\}$. Consider the element

$$
a=f_{D^{\prime}} \wedge x f_{D^{\prime}} \wedge \ldots x^{d-k-1} f_{D^{\prime}} \in \lambda^{d-k} J^{\prime} \subset \mathcal{O}_{T} \otimes \lambda^{d-k} \mathcal{O}_{D} .
$$

This clearly annihilates $J^{\prime} \subset \mathcal{O}_{T} \otimes \lambda^{1} \mathcal{O}_{D}$, so multiplication by a induces a map $\lambda^{k} \mathcal{O}_{D^{\prime}} \rightarrow \mathcal{O}_{T} \otimes \lambda^{d} \mathcal{O}_{D}$. As $f_{D^{\prime}}$ is monic of degree $k$, we see that $e^{\prime} a=e$. It follows that $x(\beta) a=b_{\beta} e^{\prime} a=b_{\beta} e$. 
On the other hand, we can expand $a$ in the form $a=\sum_{\gamma} a_{\gamma} x(\gamma)$, where $\gamma$ runs over sequences $0 \leq \gamma_{0}<\ldots<\gamma_{d-k-1}<d$. We have $x(\beta) x(\gamma)= \pm e$ if $\beta$ and $\gamma$ are related as in the statement of the proposition, and $x(\beta) x(\gamma)=0$ otherwise. It follows that $x(\beta) a= \pm a_{\gamma} e$, and thus that $b_{\beta}= \pm a_{\gamma}$.

Let $A_{\gamma}$ be the matrix whose $(i, j)$ 'th entry is the coefficient of $x^{\gamma_{j}}$ in $x^{i} f_{D^{\prime}}(x)$; it is then clear that $a_{\gamma}=\operatorname{det}\left(A_{\gamma}\right)$. On the other hand, we have $x^{i} f_{D^{\prime}}(x)=$ $\sum_{m} c_{m} x^{k+i-m}$, so $\left(A_{\gamma}\right)_{i j}=c_{k+i-\gamma_{j}}$, and the proposition follows.

\section{Thom spectra of adjoint bundles}

The following proposition is an immediate consequence of Theorem 4.6 and its proof (the first statement is just the case $k=d$ of the second statement).

Proposition 10.1 Let $V$ be a $d$-dimensional bundle over a space $X$. Then there are natural isomorphisms

$$
\begin{aligned}
\widetilde{E}^{0} \Sigma^{-d} X^{\mathfrak{u}(V)} & =\lambda_{E^{0} X}^{d} E^{0} P V \\
\widetilde{E}^{0} \Sigma^{-k} G_{k} V^{\mathfrak{u}} & =\lambda_{E^{0} X}^{k} E^{0} P V \text { for } 0 \leq k \leq d .
\end{aligned}
$$

Remark 10.2 Note that the proposition gives two different descriptions of the module $\widetilde{E}^{0} \Sigma^{-k} G_{k} V^{\mathfrak{u}}$ : the first statement with $X$ replaced by $G_{k}(V)$ and $V$ by $T$ gives

$$
\widetilde{E}^{0} \Sigma^{-k} G_{k} V^{\mathfrak{u}}=\lambda_{E^{0} G_{k} V}^{k} E^{0} P T,
$$

whereas the second statement gives

$$
\widetilde{E}^{0} \Sigma^{-k} G_{k}(V)^{\mathfrak{u}}=\lambda_{E^{0} X}^{k} E^{0} P V .
$$

We leave it to the reader to check that these two descriptions are related by the isomorphism $\phi: \lambda_{S}^{k} \mathcal{O}_{D} \rightarrow \lambda_{\operatorname{Sub}_{k}(D)}^{k} \mathcal{O}_{D^{\prime}}$ of Proposition 9.8.

In the present section we examine the isomorphisms of Proposition 10.1 more carefully. We will construct a diagram as follows, whose effect in cohomology will be identified with the diagram in Proposition 9.5.

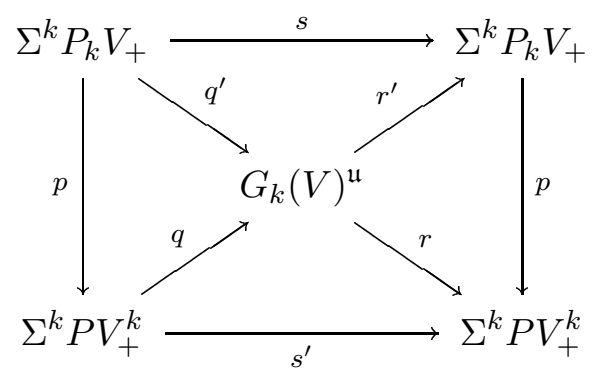

Algebraic ${ }^{3} \mathcal{G}$ Geometric Topology, Volume 2 (2002) 
Here $P V^{k}$ means the fibre product

$P V^{k}=P V \times_{X} \ldots \times_{X} P V=\left\{\left(x, L_{0}, \ldots, L_{k-1}\right) \mid x \in X, L_{0}, \ldots, L_{k-1} \in P V_{x}\right\}$.

Write $Q_{k} U(V)=F_{k} U(V) / F_{k-1} U(V)$, so that $Q_{k} U(V) \simeq G_{k} V^{\mathfrak{u}}$. As the filtration of $U(V)$ is multiplicative, the multiplication $U(V)^{k} \rightarrow U(V)$ induces a map $\left(Q_{1} U(V)\right)^{(k)} \rightarrow Q_{k} U(V)$, or equivalently $\Sigma^{k} P V_{+} \rightarrow G_{k}(V)^{\mathfrak{u}}$. This is the map $q$ in the diagram.

Recall that $P_{k} V$ is the set of points $\left(x, L_{0}, \ldots, L_{k-1}\right) \in P V^{k}$ such that the lines $L_{i}$ are mutually orthogonal. The map $p: P_{k} V \rightarrow P V^{k}$ is just the inclusion. We also have a map $P_{k} V \rightarrow G_{k} V$ sending $(x, \underline{L})$ to $\left(x, \bigoplus_{i} L_{i}\right)$, and we note that $\mathfrak{u}\left(\bigoplus_{i} L_{i}\right)$ contains $\bigoplus_{i} \mathfrak{u}\left(L_{i}\right)$. Moreover, when $L$ is one-dimensional there is a canonical isomorphism $\mathfrak{u}(L) \simeq i \mathbb{R} \simeq \mathbb{R}$, so $\bigoplus_{i} \mathfrak{u}\left(L_{i}\right) \simeq \mathbb{R}^{k}$, so we get an inclusion $\sum^{k} P_{k} V_{+} \rightarrow G_{k} V^{\mathfrak{u}}$, which we call $q^{\prime}$. It is not hard to see that this is the same as $q p$, so the left hand triangle commutes on the nose.

We next define the map $r^{\prime}: G_{k} V^{\mathfrak{u}} \rightarrow \Sigma^{k} P_{k} V_{+}$by a Pontrjagin-Thom construction. Let $N_{0}^{\prime} \subset \mathbb{R}^{k}$ be the set of sequences $\left(t_{0}, \ldots, t_{k-1}\right)$ such that $t_{0}<\ldots<t_{k-1}$, and let $N^{\prime}$ be the space of triples $(x, W, \alpha)$ where $W \in G_{k} V_{x}$ and $\alpha \in \mathfrak{u}(W)$ and $\alpha$ has $k$ distinct eigenvalues. This is easily seen to be an open subspace of the total space of the bundle $\mathfrak{u}$ over $G_{k} V$. Given such a triple, we note that the eigenvalues of $\alpha$ are purely imaginary, so we can write them as $i t_{0}, \ldots, i t_{k-1}$ with $t_{0}<\ldots<t_{k-1}$. We also put $L_{j}=\operatorname{ker}\left(\alpha-i t_{j}\right)$, so the spaces $L_{j}$ are one-dimensional and mutually orthogonal, and their direct sum is $W$. Using this we see that the map $q p: \Sigma^{k} P_{k} V_{+} \rightarrow G_{k} V^{\mathfrak{u}}$ induces a homeomorphism $N_{0}^{\prime} \times P_{k} V \rightarrow N^{\prime}$, and this gives a collapse map

$$
G_{k} V^{\mathfrak{u}} \rightarrow N^{\prime} \cup\{\infty\} \simeq\left(N_{0}^{\prime} \times P_{k} V\right) \cup\{\infty\} \simeq\left(N_{0}^{\prime} \cup\{\infty\}\right) \wedge P_{k} V_{+} .
$$

On the other hand, the inclusion $N_{0}^{\prime} \rightarrow \mathbb{R}^{k}$ gives a collapse map $S^{k} \rightarrow N_{0}^{\prime} \cup\{\infty\}$ which is a homotopy equivalence; after composing with the inverse of this, we obtain a map $G_{k} V^{\mathfrak{u}} \rightarrow \Sigma^{k} P_{k} V_{+}$, which we denote by $r^{\prime}$.

We now define a map $r: G_{k} V^{\mathfrak{u}} \rightarrow P V_{+}^{k}$. We first mimic Lemma 4.5 and define maps $m_{j}: U(1) \rightarrow U(1)$ (for $0 \leq j<k$ ) by

$$
m_{j}\left(e^{i \theta}\right)= \begin{cases}e^{i k \theta} & \text { if } j / k \leq \theta / 2 \pi \leq(j+1) / k \\ 1 & \text { otherwise }\end{cases}
$$

(where $\theta$ is assumed to be in the interval $[0,2 \pi]$ ). We then define $\delta_{k}^{\prime}: U(V) \rightarrow$ $U(V)^{k}$ by $\delta_{k}^{\prime}(g)=\left(m_{0}(g), \ldots, m_{k-1}(g)\right)$. This is homotopic to the diagonal and preserves filtrations so it induces a map $G_{k} V^{\mathfrak{u}}=Q_{k} U(V) \rightarrow Q_{k}\left(U(V)^{k}\right)$. The target of this map is the wedge of all the spaces $Q_{l_{0}} U(V) \wedge \ldots \wedge Q_{l_{k-1}} U(V)$ 
for which $\sum_{i} l_{i}=k$. We can thus project down to the factor $Q_{1} U(V) \wedge \ldots \wedge$ $Q_{1} U(V)=\Sigma^{k} P V_{+}^{k}$ to get a map $G_{k} V^{\mathfrak{u}} \rightarrow \Sigma^{k} P V_{+}^{k}$, which we call $r$.

It is not hard to recover the following more explicit description of $r$. Recall that we have a homeomorphism

$$
\gamma: U(1) \rightarrow \mathbb{R} \cup\{\infty\}
$$

given by $\gamma(z)=(z+1)(z-1)^{-1} / i$ and $\gamma^{-1}(t)=(i t+1) /(i t-1)$. One checks that $\gamma\left(e^{i \theta}\right)=-\cot (\theta / 2)$, which is a strictly increasing function of $\theta$ for $0<\theta<2 \pi$. Let $A_{j}$ denote the arc $\left\{e^{i \theta} \mid j / k<\theta / 2 \pi<(j+1) / k\right\}$, so $\gamma A_{j}$ is the interval $(-\cot (\pi j / k),-\cot (\pi(j+1) / k))$. We also define $\bar{m}_{j}=\gamma m_{j} \gamma^{-1}$, which can be regarded as a homeomorphism $\gamma A_{j} \cup\{\infty\} \rightarrow \mathbb{R} \cup\{\infty\}$, homotopy inverse to the evident collapse map in the opposite direction. If we put $N_{0}=$ $\prod_{j} \gamma A_{j} \subset N_{0}^{\prime} \subset \mathbb{R}^{k}$ then the maps $\bar{m}_{j}$ combine to give a homeomorphism $\bar{m}: N_{0} \cup\{\infty\} \rightarrow \mathbb{R}^{k} \cup\{\infty\}$, which is again homotopy inverse to the evident collapse map in the opposite direction. Now let $N \subset N^{\prime}$ be the space of triples $(x, W, \alpha)$ such that $\alpha / i$ has precisely one eigenvalue in $\gamma A_{j}$ for each $j$. If $(x, W, \alpha) \in N$ and $t_{j}$ is the eigenvalue in $\gamma A_{j}$ and $L_{j}=\operatorname{ker}\left(\alpha-i t_{j}\right)$ then we find that $\underline{t} \in N_{0}$ and $\underline{L} \in P_{k} V_{x}$ and $r(x, W, \alpha)=(\bar{m}(\underline{t}), x, \underline{L})$. On the other hand, if $(x, W, \alpha) \notin N$ we find that $r(x, W, \alpha)=\infty$. It follows that $r$ is constructed in the same way as $r^{\prime}$, except that $N^{\prime}$ and $N_{0}^{\prime}$ are replaced by the smaller sets $N$ and $N_{0}$. The projections $N^{\prime} \cup\{\infty\} \rightarrow N \cup\{\infty\}$ and $N_{0}^{\prime} \cup\{\infty\} \rightarrow N_{0} \cup\{\infty\}$ are homotopy equivalences, and it follows that $r$ is homotopic to $p r^{\prime}$. This shows that the right hand triangle in our diagram commutes up to homotopy.

We now consider the composite $s=r^{\prime} q^{\prime}: \Sigma^{k} P_{k} V_{+} \rightarrow \Sigma^{k} P_{k} V_{+}$, which is essentially obtained by collapsing out the complement of $\left(q^{\prime}\right)^{-1}\left(N^{\prime}\right)$. There is an evident action of the symmetric group $\Sigma_{k}$ on the space $\Sigma^{k} P_{k} V_{+}$, given by

$$
\sigma \cdot(\underline{t}, \underline{L})=\left(t_{\sigma^{-1}(0)}, \ldots, t_{\sigma^{-1}(k-1)}, L_{\sigma^{-1}(0)}, \ldots, L_{\sigma^{-1}(k-1)}\right) .
$$

One checks that $\left(q^{\prime}\right)^{-1}\left(N^{\prime}\right)=\coprod_{\sigma} \sigma \cdot\left(N_{0}^{\prime} \times P_{k} V\right)$, and using this one can see that $s$ is just the trace map $\operatorname{tr}_{\Sigma_{k}}=\sum_{\sigma \in \Sigma_{k}} \sigma$.

Finally, we define

$$
s^{\prime}=r q: \Sigma^{k} P V_{+}^{k} \rightarrow \Sigma^{k} P V_{+}^{k} .
$$

We can also define $\operatorname{tr}_{\Sigma_{k}}: \Sigma^{k} P V_{+}^{k} \rightarrow \Sigma^{k} P V_{+}^{k}$; we suspect that this is not the same as $s^{\prime}$, although we will see shortly that it induces the same map in cohomology. 
We now apply the functor $\widetilde{E}^{k}(-)=\widetilde{E}^{0} \Sigma^{-k}(-)$ to our diagram of spaces and write $D=D(V)$ to get the following diagram:

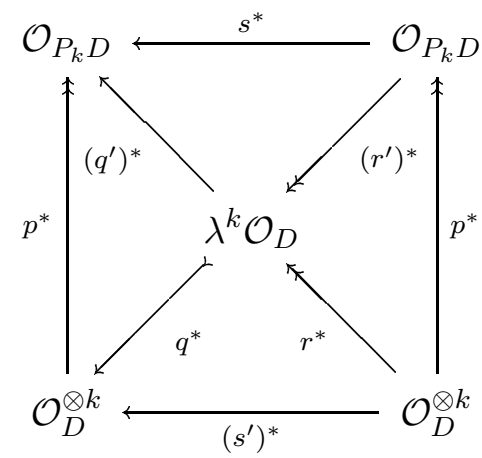

The map $p^{*}: \mathcal{O}_{D}^{\otimes k}=\mathcal{O}_{D^{k}} \rightarrow \mathcal{O}_{P_{k} D}$ is the same as considered previously; this is the definition of our identification of $\left(P_{k} V\right)_{E}$ with $P_{k} D$. It follows from the Hopf algebra isomorphism of Theorem 4.4 that $r^{*}=\mu_{k}$ and $q^{*}=\psi_{k}$, and thus that $\left(s^{\prime}\right)^{*}=\psi_{k} \mu_{k}=$ alt $_{k}$. As $\mu_{k}$ factors uniquely through $p^{*}$ we must have $\left(r^{\prime}\right)^{*}=\mu_{k}^{\prime}$. As $q^{\prime}=p q$ and $\psi_{k}^{\prime}=p^{*} \psi_{k}$ we have $\left(q^{\prime}\right)^{*}=\psi_{k}^{\prime}$. Finally, we know that $s=\operatorname{tr}_{\Sigma_{k}}$ and any permutation $\sigma \in \Sigma_{k}$ acts on the sphere $S^{k}$ with degree equal to its signature so it follows that $s^{*}=\operatorname{alt}_{k}: E^{0} P_{k} V \rightarrow E^{0} P_{k} V$.

\section{Fibrewise loop groups}

We conclude the main part of this paper by studying the fibrewise loop space $\Omega_{X} U(V)$ and thereby providing a topological realisation of the diagram in Proposition 9.6.

First, the group structure on $U(V)$ gives a group structure on $\Omega_{X} U(V)$. We also have $\Omega_{X} U(V) \simeq \Omega_{X}^{2} B U(V)$, and there is a canonical homotopy showing that a double loop space is homotopy-commutative, so the proof goes through to show that $\Omega_{X} U(V)$ is fibrewise homotopy commutative.

We next recall certain subspaces of $\Omega_{X} U(V)$ which have been considered by a number of previous authors - we will mostly refer to Crabb's exposition [2], which cites on Mitchell's paper [7] and (apparently unpublished) work of Mahowald and Richter.

Let $V$ be a vector space. Any finite Laurent series $f(z)=\sum_{i=-N}^{N} a_{i} z^{i}$ with coefficients $a_{i} \in \operatorname{End}(V)$ can be regarded as a map $U(1) \rightarrow \operatorname{End}(V)$ which we can compose with the standard homeomorphism $\gamma^{-1}: S^{1} \rightarrow U(1)$ to get a map $\hat{f}: S^{1} \rightarrow \operatorname{End}(V)$. We write $\Omega^{\text {lau }} U(V)$ for the space of based loops 
$u: S^{1} \rightarrow U(V)$ that have the form $u=\hat{f}$ for some finite Laurent series $f$, and call this the space of Laurent loops. Similarly, we write $\Omega^{\text {pol }} U(V)$ for the space of loops that have the form $\hat{f}$ for some polynomial $f$.

If $u=\hat{f}$ is a Laurent loop we have $f(z)^{-1}=f(z)^{*}=\sum_{i} a_{i}^{*} z^{-i}$ which is again a finite Laurent series. Using this we see that that $\Omega^{\text {lau }} U(V)$ is a subgroup of $\Omega U(V)$ (but $\Omega^{\text {pol }} U(V)$ is merely a submonoid). We also find that the function $d(z)=\operatorname{det}(f(z))$ is a finite Laurent series in $\mathbb{C}\left[z, z^{-1}\right]$ satisfying $d(z) \bar{d}(\bar{z})=1$ and $d(1)=1$; it follows easily that $d(z)=z^{n}$ for some integer $n$, called the degree of $u$.

Definition 11.1 (a) We write $S_{k} V$ for the space of polynomial loops of degree $k$ on $U(V)$.

(b) The product structure on $\Omega U(V)$ induces maps $S_{k} V \times S_{l} V \rightarrow S_{k+l} V$, which we call $\mu_{k l}$.

(c) Given $W \in G_{k} V$ and $z \in U(1)$ we have a polynomial $z \pi_{W}+\left(1-\pi_{W}\right) \in$ $\operatorname{End}(V)[z]$ giving rise to a based loop in $U(V)$ which we call $\phi_{k}(W)$. This defines a map $\phi_{k}: G_{k} V \rightarrow S_{k} V$. It is not hard to show that $\phi_{1}: P V \rightarrow$ $S_{1} V$ is a homeomorphism.

(d) By combining $\phi_{1}$ with the product map we get a map $\nu_{k}: P V_{X}^{k} \rightarrow S_{k} V$.

If $V$ is a bundle rather than a vector space, we make all these definitions fibrewise in the obvious way.

Note that $\nu_{k}$ induces a map $E^{*} S_{k} V \rightarrow\left(E^{*} P V\right)^{\otimes k}$, where the tensor product is taken over $E^{*} X$. We write $\operatorname{Sym}^{k}\left(E^{*} P V\right)$ for the submodule invariant under the action of $\Sigma_{k}$.

Proposition $11.2 \nu_{k}$ induces an isomorphism $E^{*} S_{k} V=\operatorname{Sym}^{k}\left(E^{*} P V\right)$, and thus an isomorphism $D(V)^{k} / \Sigma_{k} \rightarrow\left(S_{k} V\right)_{E}$.

Proof Put $d=\operatorname{dim}(V)$ and let $A$ be the set of lists $\alpha=\left(\alpha_{i} \mid i<k\right)$ with $0 \leq \alpha_{i}<d$. We have

$$
E^{*} P V_{X}^{k}=\left(E^{*} P V\right)^{\otimes k}=E^{*} \llbracket x_{i} \mid i<k \rrbracket /\left(f_{V}\left(x_{i}\right) \mid i<k\right),
$$

and the set $\left\{x^{\alpha} \mid \alpha \in A\right\}$ is a basis for this ring over $E^{*} X$. Put $A_{+}=\{\alpha \in$ $\left.A \mid \alpha_{0} \leq \ldots \leq \alpha_{k-1}\right\}$ and $M_{+}=\bigoplus_{\alpha \in A_{+}} E^{*} X$, and let $\pi: E^{*} P V_{X}^{k} \rightarrow M_{+}$be the obvious projection. This clearly induces an isomorphism $\mathrm{Sym}^{k} E^{*} P V \rightarrow$ $M_{+}$. 
We take as our basic input (proved by Mitchell [7]) the fact that when $X$ is a point, the map $\nu_{k}$ induces an isomorphism $\left(H_{*} P V\right)_{\Sigma_{k}}^{\otimes k} \rightarrow H_{*} S_{k} V$. In particular, this means that $H_{*} S_{k} V$ is a finitely generated free Abelian group, concentrated in even degrees. By duality we see that $H^{*} S_{k} V=\mathrm{Sym}^{k} H^{*} P V$, and thus that the map $\pi \nu_{k}^{*}: H^{*} S_{k} V \rightarrow \bigoplus_{A_{+}} H^{*} X$ is an isomorphism. Using an Atiyah-Hirzebruch spectral sequence we see that $\pi \nu_{k}^{*}: E^{*} S_{k} V \rightarrow \bigoplus_{A_{+}} E^{*} X$ is an isomorphism for any $E$.

Now let $X$ be arbitrary. If $V$ is trivialisable with fibre $V_{0}$ then $S_{k} V=X \times$ $S_{k} V_{0}$ and it follows from the above that $\pi \nu_{k}^{*}$ is an isomorphism. If $V$ is not trivialisable, we can still give $X$ a cell structure such that the restriction to any closed cell is trivialisable, and then use Mayer-Vietoris sequences, the five lemma, and the Milnor sequence to see that $\pi \nu_{k}^{*}$ is an isomorphism.

We next claim that the maps

$$
\mu_{k l}^{*}: E^{*} S_{k+l} V \rightarrow E^{*} S_{k} V \otimes_{E^{*} X} E^{*} S_{l} V
$$

give rise to a cocommutative coproduct. To see this, let $C(V)$ denote the following diagram:

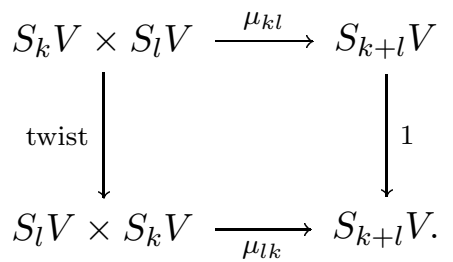

The claim is that the diagram $E^{*} C(V)$ commutes. Let $i_{0}, i_{1}: V \rightarrow V^{2}$ be the two inclusions. The map $i_{0}$ induces a map $E^{*} C\left(V^{2}\right) \rightarrow E^{*} C(V)$, and it follows easily from our previous discussion that this is surjective. It will thus be enough to show that the two ways round $E^{*} C\left(V^{2}\right)$ become the same when composed with the map

$$
\left(S_{k}\left(i_{0}\right) \times S_{l}\left(i_{0}\right)\right)^{*}: E^{*}\left(S_{k}\left(V^{2}\right) \times_{X} S_{l}\left(V^{2}\right)\right) \rightarrow E^{*}\left(S_{k} V \times_{X} S_{l} V\right) .
$$

It is standard that $i_{0}$ is homotopic to $i_{1}$ through linear isometries, so $S_{l}\left(i_{0}\right)$ is fibre-homotopic to $S_{l}\left(i_{1}\right)$. Similarly, the identity map of $S_{k+l}\left(V^{2}\right)$ is homotopic to $S_{k+l}$ (twist). It is thus enough to check that the two composites $S_{k} V \times S_{l} V \rightarrow$ $S_{k+l}\left(V^{2}\right)$ in the following diagram are the same:

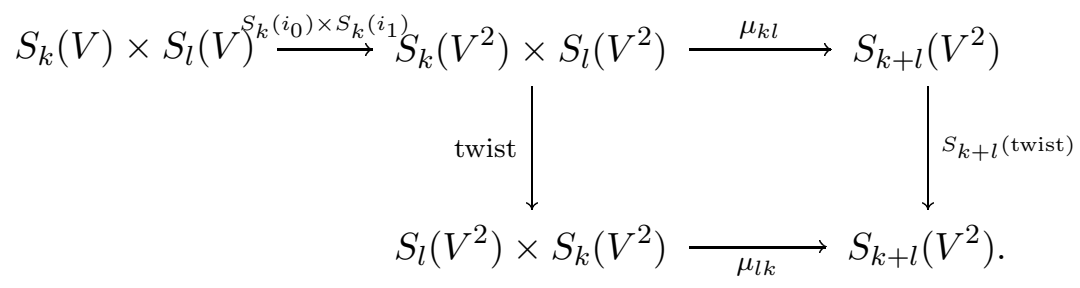

Algebraic $\mathcal{E} \mathcal{G}$ Geometric Topology, Volume 2 (2002) 
This is easy to see directly.

We now see that the map

$$
\nu_{k}^{*}: E^{*} S_{k} V \rightarrow\left(E^{*} P V\right)^{\otimes k}
$$

factors through $\operatorname{Sym}^{k}\left(E^{*} P V\right)$. As the map $\pi: \operatorname{Sym}^{k}\left(E^{*} P V\right) \rightarrow M_{+}$and its composite with $\nu_{k}^{*}$ are both isomorphisms, we deduce that $\nu_{k}^{*}: E^{*} S_{k} V \rightarrow$ $\operatorname{Sym}^{k}\left(E^{*} P V\right)$ is an isomorphism as claimed.

Corollary 11.3 The formal scheme $\left(\Omega_{X} U(V)\right)_{E}$ is the free commutative formal group over $X_{E}$ generated by the divisor $D V$.

Proof We refer to [10, Section 6.2] for background on free commutative formal groups; the results there mostly state that the obvious methods for constructing such objects work as expected under some mild hypotheses. Given a formal scheme $T$ over a formal scheme $S$, we use the following notation:

(a) $M^{+} T$ is the free commutative monoid over $S$ generated by $T$. This is characterised by the fact that monoid homomorphisms from $M^{+} T$ to any monoid $H$ over $S$ biject with maps $T \rightarrow H$ of schemes over $S$. It is clear that if there exists an $M^{+} T$ with this property, then it is unique up to canonical isomorphism. Similar remarks apply to our other definitions. In reasonable cases we can construct the colimit $\coprod_{k} T_{S}^{k} / \Sigma_{k}$ and this works as $M^{+} T$; see [10, Proposition 6.8] for technicalities.

(b) $M T$ is the free commutative group over $S$ generated by $T$.

(c) If $T$ has a specified section $z: S \rightarrow T$, then $N^{+} T$ is the free commutative monoid scheme generated by the based scheme $T$, so homomorphisms from $N^{+} T$ to $H$ biject with maps $T \rightarrow H$ such that the composite $S \stackrel{z}{\rightarrow} T \rightarrow H$ is zero. In reasonable cases $N^{+} T$ can be constructed as $\lim _{k} T_{S}^{k} / \Sigma_{k}$

(d) If $T$ has a specified section we also write $N T$ for the free commutative group over $S$ generated by the based scheme $T$.

The one surprise in the theory is that often $N T=N^{+} T$; this is analogous to the fact that a graded connected Hopf algebra automatically has an antipode. It is easy to check that $M T=\mathbb{Z} \times N T$, where $\mathbb{Z}$ is regarded as a discrete group scheme in an obvious way.

We first suppose that $V$ has a one-dimensional summand, so $V=L \oplus W$ for some bundles $L$ and $W$ with $\operatorname{dim}(L)=1$. Note that for each $x \in X$ there is a canonical isomorphism $\mathbb{C} \rightarrow \operatorname{End}(L)$ giving $U(1) \times X \simeq U(L)$. This 
gives an evident inclusion $\alpha: U(1) \times X \rightarrow U(V)$ with det $\circ \alpha=1$. We define $\beta: U(V) \rightarrow S U(V)$ by $\beta(g)=\alpha(\operatorname{det}(g))^{-1} g$, and note that $\beta(\alpha(z) g)=\beta(g)$ for all $z$.

We also have an evident map $z: X \simeq P L \rightarrow P V \simeq S_{1} V$ splitting the projection $P V \rightarrow X$. Left multiplication by $z$ gives a map $i_{k}: S_{k} V \rightarrow S_{k+1} V$. We also define $i_{k}: S_{k} V \rightarrow \Omega_{X} S U(V)$ to be the restriction of $\Omega_{X} \beta$ to $S_{k} V \subset \Omega_{X} U(V)$. Using the fact that $\beta(\alpha(z) g)=\beta(g)$ we see that $j_{k+1} i_{k}=j_{k}$. Thus, if we define $S_{\infty} V$ to be the homotopy colimit of the spaces $S_{k} V$, we get a map $j_{\infty}: S_{\infty} V \rightarrow$ $\Omega_{X} S U(V)$ of spaces over $X$. Using the usual bases for $E^{*} S_{k} V=\operatorname{Sym}^{k}\left(E^{*} P V\right)$ we find that the maps $i_{k}^{*}: \operatorname{Sym}^{k+1}\left(E^{*} P V\right) \rightarrow \operatorname{Sym}^{k}\left(E^{*} P V\right)$ are surjective. It follows using the Milnor sequence that $E^{*} S_{\infty} V=\lim _{k} \operatorname{Sym}^{k}\left(E^{*} P V\right)$ and thus that $\left(S_{\infty} V\right)_{E}=\lim _{k} D V^{k} / \Sigma_{k}$. We claim that this is the same as $N^{+} D V$; this is clear modulo some categorical technicalities, which are covered in $[10$, Section 6.2]. In the case where $X$ is a point, it is well-known and easy to check (by calculation in ordinary homology) that the map $S_{\infty} V \rightarrow \Omega_{X} S U(V)$ is a weak equivalence. In the general case we have a map between fibre bundles that is a weak equivalence on each fibre; it follows easily that the map is itself a weak equivalence, and thus that $\Omega_{X} S U(V)_{E}=N^{+} D V$. On the other hand, as $\Omega_{X} S U(V)$ is actually a group bundle, we see that $\Omega_{X} S U(V)_{E}$ is a formal group scheme, so $N^{+} D V=N D V$.

We now turn to the groups $\Omega_{X} U(V)$. We define $\mathbb{Z}_{X}=\mathbb{Z} \times X$, viewed as a bundle of groups over $X$ in the obvious way. This can be identified with $\Omega_{X}(U(1) \times$ $X)$ so the determinant map gives rise to a homomorphism $\delta: \Omega_{X} U(V) \rightarrow$ $\mathbb{Z}_{X}$. Given $(n, x) \in \mathbb{Z}_{X}$ we have a homomorphism $U(1) \rightarrow U\left(V_{x}\right)$ given by $z \mapsto \alpha\left(z^{n}\right)$. This construction gives us a map $\sigma: \mathbb{Z}_{X} \rightarrow \Omega_{X} U(V)$ with $\delta \sigma=$ 1 and thus a splitting $\Omega_{X} U(V) \simeq \mathbb{Z} \times \Omega_{X} S U(V)$ and thus an isomorphism $\Omega_{X} U(V)_{E}=\mathbb{Z} \times N D V=M D V$. One can check that the various uses of the map $\alpha$ cancel out and that the standard inclusion $D V \rightarrow M D V$ is implicitly identified with the map coming from the inclusion $P V=S_{1} V \rightarrow \Omega_{X} U(V)$. This proves the corollary in the case where $V$ has a one-dimensional summand.

Now suppose that $V$ does not have such a summand. We have an evident coequaliser diagram $P V \times_{X} P V \Longrightarrow P V \rightarrow X$, giving rise to a coequaliser diagram $D V \times_{X_{E}} D V \rightarrow D V \rightarrow X_{E}$ of schemes over $X_{E}$, in which the map $D V \rightarrow X_{E}$ is faithfully flat. The pullback of $V$ to $P V$ has a tautological onedimensional summand, which implies that $\left(P V \times_{X} \Omega_{X} U(V)\right)_{E}$ has the required universal property in the category of formal group schemes over $P V_{E}$. Similar remarks apply to $P V \times_{X} P V \times_{X} \Omega_{X} U(V)$. It follows by a descent argument that $\Omega_{X} U(V)$ itself has the required universal property, as one sees easily from [10, 
Proposition 2.76 and Remark 4.52].

We next recall the standard line bundle over $S_{k} V$, which we will call $T$; see $[2,7]$ for more details. Write $A=\mathbb{C}[z]$ and $K=\mathbb{C}\left[z, z^{-1}\right]$. A point of $S_{k} V$ has the form $(x, \hat{f})$ for some $f \in \operatorname{End}_{\mathbb{C}}\left(V_{x}\right)[z] \simeq \operatorname{End}_{A}\left(A \otimes V_{x}\right)$. Multiplication by $f$ defines a surjective endomorphism $m(f)$ of $(K / A) \otimes V$, and we define $T_{(x, f)}$ to be the kernel of this endomorphism. One can check that this always has dimension $k$ over $\mathbb{C}$ and that we get a vector bundle. This is classified by a map $\tau_{k}: S_{k} V \rightarrow B U(k) \times X$ of spaces over $X$. It is easy to see that the restriction of $T$ to $G_{k} V \subset S_{k} V$ is just the tautological bundle.

There are evident short exact sequences

$$
\operatorname{ker}(m(g)) \longrightarrow \operatorname{ker}(m(f g)) \stackrel{m(g)}{\rightarrow} \operatorname{ker}(m(f)),
$$

which can be split using the inner products to give isomorphisms $\mu_{k l}^{*} T \simeq \pi_{0}^{*} T \oplus$ $\pi_{1}^{*} T$ over $S_{k} V \times_{X} S_{l} V$. This means that the map $\tau: \coprod_{k} S_{k} V \rightarrow\left(\coprod_{k} B U(k)\right) \times$ $X$ is a homomorphism of $H$-spaces over $X$.

We now have a diagram of spaces as follows:

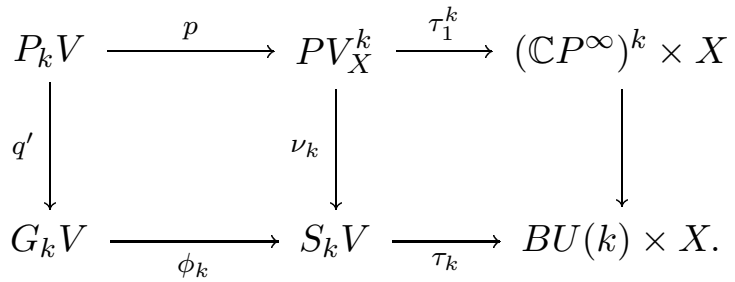

It is easy to identify the corresponding diagram of schemes with the diagram of Proposition 9.6.

\section{A Appendix : Functional calculus}

In this appendix we briefly recall some basic facts about functional calculus for normal operators. An endomorphism $\alpha$ of a vector space $V$ is normal if it commutes with its adjoint. For us the relevant examples are Hermitian operators (with $\alpha^{*}=\alpha$ ), anti-Hermitian operators (with $\alpha^{*}=-\alpha$ ) and unitary operators (with $\alpha^{*}=\alpha^{-1}$ ).

For any operator $\alpha$ and any $\lambda \in \mathbb{C}$ we have

$$
\operatorname{ker}(\alpha-\lambda)^{\perp}=\operatorname{image}\left((\alpha-\lambda)^{*}\right)=\operatorname{image}\left(\alpha^{*}-\bar{\lambda}\right) .
$$


If $\alpha$ is normal we deduce that $\operatorname{ker}(\alpha-\lambda)^{\perp}$ is preserved by $\alpha$, and it follows easily that $V$ is the orthogonal direct sum of the eigenspaces of $\alpha$. It follows in turn that the operator norm of $\alpha$ (defined by $\|\alpha\|=\sup \{\|\alpha(v)\|:\|v\|=1\}$ ) is just the same as the spectral radius (defined as the maximum absolute value of the eigenvalues of $\alpha$ ).

Now let $X$ be a subset of $\mathbb{C}$ containing the eigenvalues of $\alpha$, and let $f: X \rightarrow \mathbb{C}$ be a continuous function. We define $f(\alpha)$ to be the endomorphism of $V$ that has eigenvalue $f(\lambda)$ on the space $\operatorname{ker}(\alpha-\lambda)$. From this definition it is clear that the following equations are valid whenever they make sense:

$$
\begin{aligned}
c(\alpha) & =c .1_{V} \text { if } c \text { is constant } \\
\operatorname{id}(\alpha) & =\alpha \\
\operatorname{Re}(\alpha) & =\left(\alpha+\alpha^{*}\right) / 2 \\
\operatorname{Im}(\alpha) & =\left(\alpha-\alpha^{*}\right) /(2 i) \\
(f+g)(\alpha) & =f(\alpha)+g(\alpha) \\
(f g)(\alpha) & =f(\alpha) g(\alpha) \\
\bar{f}(\alpha) & =f(\alpha)^{*} \\
(f \circ g)(\alpha) & =f(g(\alpha)) \\
\|f(\alpha)\| & \leq \sup _{x \in X}|f(x)| .
\end{aligned}
$$

The continuity properties of $f(\alpha)$ are less clear from our definition. However, they are provided by the following result.

Proposition A.1 Let $X$ be a closed subset of $\mathbb{C}$, and $V$ a vector space. Let $N(X, V)$ be the set of normal operators on $V$ whose eigenvalues lie in $X$, and let $C(X, \mathbb{C})$ be the set of continuous functions from $X$ to $\mathbb{C}$ (with the topology of uniform convergence on compact sets). Define a function $E: C(X, \mathbb{C}) \times$ $N(X, V) \rightarrow \operatorname{End}(V)$ by $E(f, \alpha)=f(\alpha)$. Then $E$ is continuous.

Proof Let $A$ be the set of functions $f \in C(X, \mathbb{C})$ for which the function $\alpha \mapsto f(\alpha)$ is continuous. Using the above algebraic properties, we see that $A$ is a subalgebra of $C(X, \mathbb{C})$ containing the functions $z \mapsto \operatorname{Re}(z)$ and $z \mapsto \operatorname{Im}(z)$. By the Stone-Weierstrass theorem, it is dense in $C(X, \mathbb{C})$. Now suppose we have $f \in C(X, \mathbb{C}), \alpha \in N(X, V)$ and $\epsilon>0$. Put $Y=\{x \in X|| x \mid \leq\|\alpha\|+1\}$, which is compact. As $A$ is dense we can choose $p \in A$ with $|f-p|<\epsilon / 4$ on $Y$. As $p \in A$ can choose $\delta$ such that $\|p(\beta)-p(\alpha)\|<\epsilon / 4$ whenever $\|\beta-\alpha\|<\delta$. 
We may also assume that $\delta<1$, which means that when $\|\beta-\alpha\|<\delta$ we have $\beta \in Y$. Now if $|f-g|<\epsilon / 4$ on $Y$ and $\|\alpha-\beta\|<\delta$ then

$$
\begin{gathered}
\|f(\alpha)-g(\beta)\| \leq \\
\|f(\alpha)-p(\alpha)\|+\|p(\alpha)-p(\beta)\|+ \\
\|p(\beta)-f(\beta)\|+\|f(\beta)-g(\beta)\| \\
<\epsilon / 4+\epsilon / 4+\epsilon / 4+\epsilon / 4=\epsilon,
\end{gathered}
$$

as required.

The following proposition is an elementary exercise in linear algebra.

Proposition A.2 Let $\alpha: V \rightarrow W$ be a linear map. Then $\alpha^{*} \alpha$ and $\alpha \alpha^{*}$ are self-adjoint endomorphisms of $V$ and $W$ with nonnegative eigenvalues. For each $t>0$ the map $\alpha$ gives an isomorphism of $\operatorname{ker}\left(\alpha^{*} \alpha-t\right)$ with $\operatorname{ker}\left(\alpha \alpha^{*}-t\right)$, so the nonzero eigenvalues of $\alpha^{*} \alpha$ and their multiplicities are the same as those of $\alpha \alpha^{*}$. If $f:[0, \infty) \rightarrow \mathbb{R}$ then $\alpha \circ f\left(\alpha^{*} \alpha\right)=f\left(\alpha \alpha^{*}\right) \circ \alpha$.

Definition A.3 We write $w(V)=\left\{\alpha \in \operatorname{End}(V) \mid \alpha^{*}=\alpha\right\}$ (the space of self-adjoint endomorphisms of $V$ ). If $\alpha \in w(V)$ then the eigenvalues of $\alpha$ are real, so we can list them in descending order, repeated according to multiplicity. We write $e_{k}(\alpha)$ for the $k$ 'th element in this list, so $e_{1}(\alpha) \geq \ldots \geq e_{n}(\alpha)$ and $\operatorname{det}(t-\alpha)=\prod_{k}\left(t-e_{k}(\alpha)\right)$.

We will need the following standard result:

Proposition A.4 The functions $e_{k}: w(V) \rightarrow \mathbb{R}$ are continuous.

Proof Let $\gamma$ be a simple closed curve in $\mathbb{C}$ and let $m$ be an integer. Let $U$ be the set of endomorphisms of $V$ that have precisely $m$ eigenvalues (counted according to multiplicity) inside $\gamma$, and no eigenvalues on $\gamma$. A standard argument with Rouché's theorem shows that $U$ is open in $\operatorname{End}(V)$.

Given real numbers $r \leq R$, consider the rectangular contour $\gamma_{r, R}$ with corners at $r \pm i$ and $R \pm i$. Clearly $e_{k}(\alpha)>r$ iff $\alpha$ has at least $k$ eigenvalues inside $\gamma_{r, R}$ for some $R$. It follows that $\left\{\alpha \mid e_{k}(\alpha)>r\right\}$ is open, as is $\left\{\alpha \mid e_{k}(\alpha)<r\right\}$ by a similar argument. This implies that $e_{k}$ is continuous. 


\section{References}

[1] P Bressler, S Evens, Schubert calculus in complex cobordism, Trans. Amer. Math. Soc. 331 (1992) 799-813

[2] M C Crabb, On the stable splitting of $\mathrm{U}(n)$ and $\Omega \mathrm{U}(n)$, from: "Algebraic topology, Barcelona, 1986", Springer, Berlin (1987) 35-53

[3] M Crabb, I James, Fibrewise homotopy theory, Springer-Verlag London Ltd., London (1998)

[4] A Grothendieck, Sur quelques propriétés fondamentales en théorie des intersections, Séminaire C. Chevalley, Ecole Normale Supérieure 2 (1958)

[5] N Kitchloo, Cohomology splittings of Stiefel manifolds (1999), preprint

[6] H Miller, Stable splittings of Stiefel manifolds, Topology 24 (1985) 411-419

[7] S A Mitchell, A filtration of the loops on $\mathrm{SU}(n)$ by Schubert varieties, Math. Z. 193 (1986) 347-362

[8] D G Northcott, Finite free resolutions, Cambridge University Press, Cambridge (1976), cambridge Tracts in Mathematics, No. 71

[9] P Pragacz, Enumerative geometry of degeneracy loci, Ann. Sci. École Norm. Sup. (4) 21 (1988) 413-454

[10] N P Strickland, Formal schemes and formal groups, from: "Homotopy invariant algebraic structures (Baltimore, MD, 1998)", Amer. Math. Soc., Providence, RI (1999) 263-352

Department of Mathematics, University of Sheffield

Western Bank, Sheffield, S10 2TN,UK

Email: N.P.Strickland@sheffield.ac.uk

Received: 3 April 2001 Revised: 5 November 2002 